\title{
Analog Photonic Fractional Signal Processing
}

\author{
Christian Cuadrado-Laborde*t, ${ }^{*}$, Luis Poveda-Wong*, \\ Antonio Carrascosa*, José L. Cruz*, Antonio Díez*, Miguel V. Andrés* \\ *ICMUV, Universidad de Valencia, Burjassot, Spain \\ †Instituto de Física Rosario (CONICET-UNR), Rosario, Argentina \\ ${ }^{\ddagger}$ Pontificia Universidad Católica Argentina, Facultad de Química e Ingeniería, Rosario, Argentina
}

\section{Contents}

1. Introduction 94

2. Fractional Calculus 97

2.1 Definition and Historical Overview 97

$\begin{array}{lr}2.2 \text { Photonic Fractional Differentiation } & 100\end{array}$

$\begin{array}{ll}2.3 \text { Photonic Fractional Integration } & 134\end{array}$

2.4 Uses and Applications of Photonics Fractional Calculus Operators 137

3. Fractional Hilbert Transform 144

3.1 Definition and Historical Overview 144

3.2 Photonic Fractional Hilbert Transform 146

$\begin{array}{ll}3.3 \text { Present and Future Perspectives } & 161\end{array}$

4. Fractional Fourier Transform 162

4.1 Definition and Historical Overview 162

4.2 Photonic Fractional Fourier Transform 164

4.3 Uses and Applications of Photonic Fractional Fourier Transform 168

5. Conclusions and Outlook 171

$\begin{array}{ll}\text { Acknowledgments } & 172\end{array}$

References 173

\begin{abstract}
In this work, we provide an up to date overview on the subject of photonic fractional signal processing, including both, in-fiber and waveguide on-chip technology. Thus, we discuss in detail fractional differentiation, fractional integration, fractional Hilbert transforms, and finally, fractional Fourier transforms. In each case, the underlying mathematical principles are explained for each operation, together with a short historical discussion in the context of classical optics. After that, the different proposals to perform these operations photonically on the complex field envelope of a given light pulse are
\end{abstract}


presented, divided according to its working principle. Finally, current applications for these operators are also discussed, as well as some of its future possibilities are envisaged.

Keywords: Photonics, Signal processing, Fractional operations, Optical fiber, Waveguide on-chip

\section{INTRODUCTION}

Nowadays, signal processing in optical networks is implemented based on digital signal processing. This involves several well-defined steps: opticalto-electrical conversion, electronic sampling, digital signal processing, and back electrical-to-optical conversion. There is a clear bottleneck in this sequence: the electronic sampling rate. A solution to circumvent this problem is to perform the signal processing directly in the optical domain using a photonic signal processor. This involves some kind of light manipulation; e.g., in the form of a well-defined spectral filtering. Therefore, not only does it obviously avoid the optical-electrical conversion and vice versa, but, even more important, the electronic sampling. The key point is that a photonic signal processor has the ability to perform a certain operation directly on the complex field envelope of a given light signal. Furthermore, as in their electronic counterpart, this operation can be performed analogically or digitally. In this work, we will refer exclusively to the former.

So far several analog photonic signal processors have been reported based on fiber-optics or waveguide on-chip technology. Each solution has pros and cons. Between the advantages of in-fiber solutions, we have: inherent full compatibility with fiber-optics networks, simplicity, cost effectiveness, low insertion loss, and (in some cases) polarization independence. On the other hand, advantages of waveguides on-chip solutions are a much smaller foot-print (best integrability) and power efficiency. Unfortunately they also have much more power insertion losses. This fact necessitates a light power amplifier stage either at the input or at the output, or even at both ports.

A photonic signal processor can be used to perform a given, relatively simple task, on a given light pulse. In a sense, it should be thought of as a basic building block, of which a combination can accomplish more sophisticated tasks. Among them, we can mention differentiation, integration, and several transforms (such as Hilbert or Fourier transforms). The possible applications for these devices encompass optical pulse shaping, optical computing, information processing systems, and ultrahigh-speed coding, among 
other uses. Thus, both in-fiber and waveguide on-chip solutions have been presented to perform different mathematical tasks, but always focusing in the integer-order (also known as ordinary) operation.

However, fractional-or more properly, noninteger-operations play an important role in information processing. They offer a new degree of freedom - the fractional order-over which to optimize, which can be used for a better characterization of a signal under test, or as an additional encoding parameter. Every mathematical operation outlined in the preceding paragraph has been generalized to a fractional degree. As an example, the fractional Fourier transform (FFT) has found widespread use for signal processing purposes and is now routinely used (Ozaktas, Zalevsky, \& Kutay, 2001). Furthermore, few people knew (including researchers) that the concept of fractional calculus is as old as their integer counterpart. This calculus branch is far from being a sterile exercise in pure mathematics, since many problems ranging from physics to economics, and engineering to material science, have been successfully solved by using fractional calculus operations (Das, 2011; Sabatier, Agrawal, \& Tenreiro Manchado, 2007). From a more fundamental point of view, we turn back to the old question: nature follows mathematics ... but which? Further, why should nature be restricted to integer-order operations? Our aim in this work is to translate to the photonic domain the flexibility provided by fractional mathematical operations.

To the best of our knowledge, our works were the first to discuss the possibility to perform a photonic fractional differentiation (PFD), photonic fractional integration (PFI), and photonic fractional Hilbert transform (PFHT). In the case of the photonic fractional Fourier transform (PFFT), we were the first to experimentally demonstrate its viability. We were also the first to find a specific application for a PFD, the phase recovery of a given light pulse. Now, almost 10 years after our first contribution on the subject, it is very rewarding to look back and see how many colleagues have joined this fascinating field of study. Our intention here is to provide an up to date overview of the subject of photonic fractional signal processing, not only from our own contributions on the subject, but including important works of other research teams. As a team, our know-how involves the use of fiberoptics technology; therefore, our own proposals were focused on in-fiber devices. However, we have decided to include waveguide on-chip technology to provide a more complete state-of-the-art review of this subject.

This review is organized as follows: Section 1 was the introduction. Section 2 is devoted to fractional calculus. In Section 2.1, we provide the 
mathematical definitions, together with a short introduction on the subject. Afterward, we begin with PFD in Section 2.2, which in turn is divided according to the several proposals found in the literature. In Section 2.3, we describe the far less visited topic of PFI. In Section 2.4, we describe in some detail two different applications for PFD and PFI. The fractional Hilbert transform (FHT) is visited in Section 3. We provide its mathematical definition, together with a short introduction on the subject, in Section 3.1. PFHT has been an active field of research; for this reason, we have subdivided the different proposals according to its operating principle (Section 3.2). Finally, in Section 4, we study the FFT. After a short historical introduction and mathematical definition (Section 4.1), we describe in Section 4.2, the different approaches to PFFT, divided according to the physical operating principle. We conclude this review in Section 5, where we outline the conclusions of this work. Finally, a complete list of acronyms used throughout this work is provided in Table 1.

Table 1 List of Acronyms

\begin{tabular}{ll}
\hline APS-FBG & Asymmetrical pi-phase shifted fiber Bragg grating \\
\hline CMB & Core-mode blocker \\
\hline CMOS & Complementary metal-oxide semiconductor \\
\hline DCF & Dispersion compensation fiber \\
\hline DLP & Discrete layer peeling \\
\hline EDFA & Erbium-doped fiber amplifier \\
\hline FBG & Fiber Bragg grating \\
\hline FHT & Fractional Hilbert transform \\
\hline FFT & Fractional Fourier transform \\
\hline GRIN & Graded index media \\
\hline HT & Hilbert transform \\
\hline LPG & Long-period grating \\
\hline MMI & Multimode interference \\
\hline MRR & Microring resonator \\
\hline MZI & Mach-Zehnder interferometer \\
\hline OCT & Optical coherence tomography \\
\hline
\end{tabular}


Table 1 List of Acronyms-cont'd

\begin{tabular}{ll}
\hline OFC & Optical fiber coupler \\
\hline PC & Polarization controller \\
\hline PFD & Photonic fractional differentiation \\
\hline PFFT & Photonic fractional Fourier transform \\
\hline PFHT & Photonic fractional Hilbert transform \\
\hline PFI & Photonic fractional integration \\
\hline PM & Phase modulator \\
\hline SMF & Single-mode fiber \\
\hline SOI & Silicon-on-isolator \\
\hline TFBG & Tilted fiber Bragg gratings \\
\hline
\end{tabular}

\section{FRACTIONAL CALCULUS}

\subsection{Definition and Historical Overview}

The $n$ th-order fractional integral of $f(t)$ is defined by (Miller \& Ross, 1993; Oldham \& Spanier, 1974):

$$
\frac{\mathrm{d}^{-n}}{\mathrm{~d} t^{-n}} f(t) \equiv \frac{1}{\Gamma(n)} \int_{0}^{t}(t-\xi)^{n-1} f(\xi) \mathrm{d} \xi
$$

where $\Gamma(n)$ is the gamma function and $n \in \mathbb{R}^{+}$. A short digression is in order here relating to a convention in the notation used in the mathematical literature in this field. Since differentiation and integration are generally inverse operations; the symbol of one of them was chosen (differentiation) to represent both; thus, a negative number in the differential symbol represents integration. Of course, a positive number in the differential symbol represents, as usual, a differentiation. By the way, for the generalized operator $\mathrm{d}^{ \pm n} / \mathrm{d} t^{ \pm n}$ was coined the term "differintegral" (Oldham \& Spanier, 1974; Sabatier et al., 2007). The denomination of fractional for this operation is formally incorrect, since $n$ can be real; however, it is preserved because of historical reasons. Eq. (1) is a special case-with the lower integration limit equal to zero- of the more general Riemann-Liouville operator. 
Fractional differentiation of order $m$ is defined through an integer-order differentiation of degree $p$, of a fractional integration of degree $n$, as follows (Oldham \& Spanier, 1974):

$$
\frac{\mathrm{d}^{m}}{\mathrm{~d} t^{m}} f(t)=\frac{\mathrm{d}^{p}}{\mathrm{~d} t^{p}}\left[\frac{\mathrm{d}^{-n}}{\mathrm{~d} t^{-n}} f(t)\right]
$$

with $m \in \mathbb{R}^{+}, n=p-m$, and $p \in \mathbb{Z}^{+}$. For example, an $m=0.3$ order fractional differentiation of $f(t)$ can be performed by a $p=1$ order differentiation of an $n=0.7$ order fractional integration of $f(t)$. This representation of fractional differentiation based on fractional integration-in turn defined in Eq. (1) — will be useful in the following, when we discuss the nonlocal properties of fractional differentiation.

Unfortunately, neither fractional integration nor fractional differentiation has a simple geometrical interpretation along the line of an area under a curve or the slope of its tangent, respectively, as in its integer-order counterpart. However, we can gain some insight into fractional integration by considering Eq. (1) in a more general sense, as a causal convolution integral:

$$
g(t)=\int_{0}^{t} h_{n}(t-\xi) f(\xi) \mathrm{d} \xi
$$

which transforms the input signal $f(t)$ into the output signal $g(t)$ through the memory function-impulse response- $h_{n}(t)$. Now, let $h_{n}(t)=\Phi(t)$ - with $\Phi(t)$ the Heaviside step function-then we have an ordinary first-order integration. On the contrary, if $h_{n}(t)=\delta(t)$-with $\delta(t)$ the Dirac delta function - then there is no transformation at all, i.e., $g(t)=f(t)$, and we have a zeroth-order integration. Now, the Riemann-Liouville's memory function, see Eq. (1), which we rewrite below:

$$
h_{n}(t)=t^{n-1} / \Gamma(n),
$$

interpolates, in a certain sense, between the step Heaviside $(n \rightarrow 1)$ and Dirac's delta functions $(n \rightarrow 0)$ (see Fig. 1). In the sense that a strong weight is assigned to the current value of the signal in the convolution mechanism, i.e., $h(t) \rightarrow \infty$ when $t \rightarrow 0$, but unlike the delta function, its memory is not limited to the current value but distributed over the whole support of $h_{n}(t)$, albeit not evenly (Rutman, 1995). Thus, one arrives to a rather unexpected conclusion: fractional differentiation is nonlocal, its value depends on past values and it is not totally determined by an arbitrarily small neighborhood of it. When the differentiation order tends to an integer 


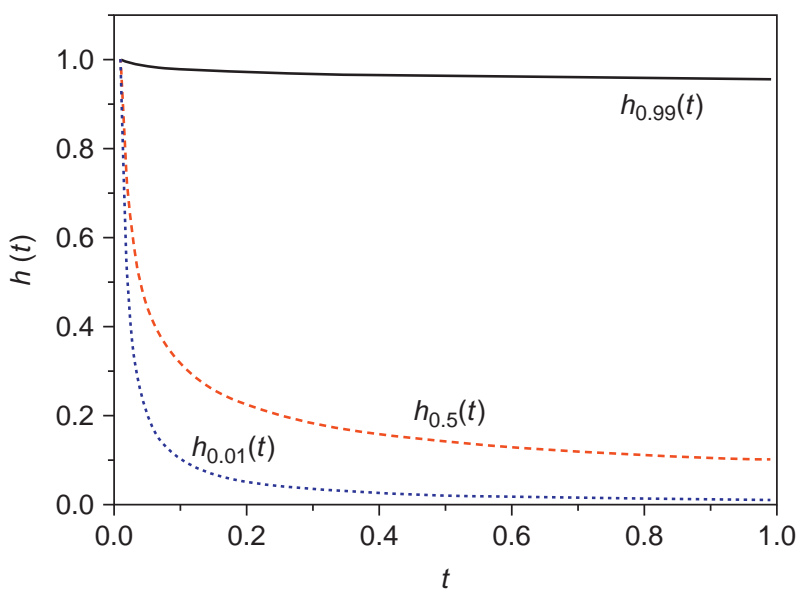

Fig. 1 Plot of the fractional integral kernel $h_{n}(t)$ (normalized), for three different values of $n=0.99,0.5$, and 0.01 .

number, this nonlocal behavior collapses to the usual local one. Therefore, in generalized differentiation, locality is the exception rather than the rule. Merely as an example, complex systems having nonlocal dynamics involving long memory in time have been modeled with fractional calculus operators (Sabatier et al., 2007).

Fortunately, both fractional differentiation and fractional integration can be performed more or less straightforwardly in the Fourier domain. Let $F(\omega)$ be the Fourier transform of the temporal signal $f(t)$, where $\omega$ is the baseband frequency, i.e., $\omega=\omega_{\text {opt }}-\omega_{0}$, with $\omega_{\text {opt }}$ the optical frequency and $\omega_{0}$ the carrier optical frequency of the signal. Whereas the spectral characteristics of $h_{n}(t)$, see Eqs. (1) and (3), are determined by its Fourier transform: $(-j \omega)^{-n}$, with $j=\sqrt{-1}$. Now, and according to the convolution theorem, the Fourier transform of the fractional integral can be obtained through:

$$
\mathfrak{I}\left[\frac{\mathrm{d}^{-n}}{\mathrm{~d} t^{-n}} f(t)\right]=(-j \omega)^{-n} F(\omega)
$$

On the other hand, by applying Eq. (4) into Eq. (2), the Fourier domain version for fractional differentiation results in:

$$
\mathfrak{I}\left[\frac{\mathrm{d}^{n}}{\mathrm{~d} t^{n}} f(t)\right]=(-j \omega)^{n} F(\omega)
$$

Therefore, both fractional integration and fractional differentiation can be optically implemented by optimally filtering in the Fourier domain by 
applying the filters defined by Eqs. (5) and (6), respectively. In both cases, this implies a spectral behavior with a $n \times \pi$ phase discontinuity at $\omega=0$, and a $|\omega|^{-n}\left(|\omega|^{n}\right)$ dependence for the amplitude of the transfer function for fractional integration (differentiation). Essentially, in amplitude, a low-pass filtering is required for fractional integration, and high-pass filtering for fractional differentiation.

In space optics, fractional integration/differentiation can be performed with a $4 f$ imaging system. An object with a complex-amplitude transmittance is located in the input plane and the filter function $(-j \omega)^{ \pm n}$ in the Fourier plane; i.e., at $2 f$. As a result, in the output plane located at $4 f$, we obtain the complex amplitude of the $n$ th-order fractional integration/ differentiation of the input. The optically calculated images are reversed with respect to mathematical formulas, since the periodicity of the Fourier transform is not twice, but fourfold. However, in most cases, this change of sign in the transversal spatial coordinates is irrelevant (Lancis, Szoplik, Tajahuerce, Climent, \& Fernández-Alonso, 1997).

\subsection{Photonic Fractional Differentiation}

\subsubsection{The Mach-Zehnder Interferometry Approach}

By PFD we mean a device that provides, at its output, a $n$ th-order time derivative of the complex field envelope of an arbitrary input optical waveform. As far as we know, the first time that the possibility to perform a PFD was discussed was in 2008. Then Cuadrado-Laborde (2008) demonstrated that a fiber-optics-based Mach-Zehnder interferometer (MZI) indeed performs a fractional differentiation on an input light pulse, whenever the power-splitting ratios were adequately selected. Previously, Li et al. (2006) also theoretically discussed the use of an in-fiber MZI, but they focused on integer-order differentiation.

Fig. 2 shows schematically the setup proposed for fractional differentiation; it is a MZI, exemplified through two fiber-optic couplers, each one characterized by its power-splitting ratio $\rho_{i}$, with $0 \leq \rho_{i} \leq 1$, and $i=1$, 2. It is worth to mention that the development of interferometers with fiberoptic couplers has at present some shortcomings related with the stability and the necessity of accurate delay line control. For these reasons, as we will see later, this proposal was first experimentally implemented in an integrated waveguide platform, and later in an in-fiber modal interferometer. In Fig. 2, both arms have the same mode-propagation constants $\beta$, but different lengths $L_{i}$. By the transfer matrix formalism, it is easy to show 


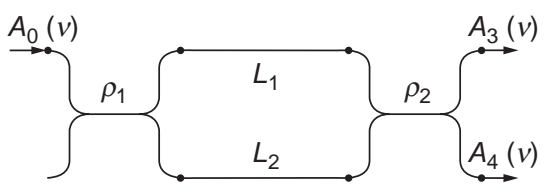

Fig. 2 Scheme of the proposed photonic device for implementing a fractional differentiator; $\rho_{1}$ and $\rho_{2}$ are the power-splitting ratios, $L_{1}$ and $L_{2}$ are the fiber lengths between couplers, $A_{0}(\nu)$ represents the input to the setup, whereas $A_{3}(\nu)$ and $A_{4}(\nu)$ represent the bar and cross-optical outputs, respectively, in the spectral domain, with $\nu=\omega / 2 \pi$ as the baseband optical frequency. From Cuadrado-Laborde, C. (2008). Alloptical ultrafast fractional differentiator. Optical and Quantum Electronics, 40, 983-990. https://doi.org/10.1007/s11082-009-9282-5.

that the transfer functions for the bar $(B)$ and cross $(C)$ ports are given by (Agrawal, 2001):

$$
\begin{aligned}
& B(\nu) \equiv A_{3}(\nu) / A_{0}(\nu)=1-\chi \exp (j 2 \pi \nu \tau), \\
& C(\nu) \equiv A_{4}(\nu) / A_{0}(\nu)=1+\eta \exp (j 2 \pi \nu \tau),
\end{aligned}
$$

where $\tau=\left(L_{2}-L_{1}\right) \nu_{g}^{-1}$ is the extra group delay in the longer arm of the MZI, $A_{i}(\nu)$ represents the amplitude of the corresponding signal in the spectral domain, $\nu_{g}$ is the group velocity; whereas the preexponential factors $\chi$ and $\eta$ are related with the power-splitting ratios through:

$$
\begin{gathered}
\chi^{2}=\frac{\left(1-\rho_{1}\right)\left(1-\rho_{2}\right)}{\rho_{1} \rho_{2}}, \\
\eta^{2}=\frac{\left(1-\rho_{1}\right) \rho_{2}}{\left(1-\rho_{2}\right) \rho_{1}} .
\end{gathered}
$$

The bar port transfer function $B(\nu)$ shows phase changes periodically at frequencies given by $\nu_{\mathrm{B}}=\kappa \tau^{-1}$, with $\kappa \in \mathbb{Z}$. These phase changes $\Delta \theta_{\mathrm{B}}$ increase and become more abrupt as $\chi$ rises from 0 to 1 . The relationship between $\chi$ and $\Delta \theta_{\mathrm{B}}$ can be obtained by finding the maximum (or minimum) of the function $\arg [B(\nu)]$ by using Eqs. (7) and (9), resulting in:

$$
\chi=\frac{\tan \left(\Delta \theta_{\mathrm{B}} / 2\right)}{\sqrt{\tan ^{2}\left(\Delta \theta_{\mathrm{B}} / 2\right)+1}}
$$

Fig. 3 shows the relationship between $\Delta \theta_{\mathrm{B}}$ and the preexponential factor $\chi$. At $\chi=1$ there is an abrupt phase change of $\Delta \theta_{\mathrm{B}}=\pi$, whereas the spectral response in amplitude behaves linearly as $|\nu|$ (at first-order approximation in $\nu$; see Eq. 6); which is precisely the behavior demanded on a first-order differentiator (Park, Azaña, \& Slavík, 2007). The cross port transfer function 


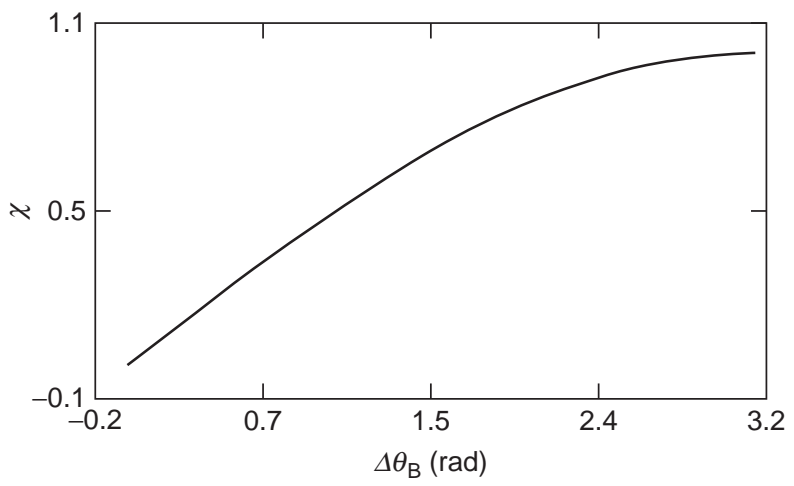

Fig. 3 Preexponential factor $\chi$ as a function of the phase change $\Delta \theta_{\mathrm{B}}$. From CuadradoLaborde, C. (2008). All-optical ultrafast fractional differentiator. Optical and Quantum Electronics, 40, 983-990. https://doi.org/10.1007/s11082-009-9282-5.

$C(\nu)$, see Eq. (8), shows an identical behavior to $B(\nu)$ (when $\eta=\chi$ ), but at shifted optical frequencies given by $\omega_{C}=2 \pi(\kappa+1 / 2) \tau^{-1}$, with a relationship between $\eta$ and $\Delta \theta_{\mathrm{C}}$ identical to Eq. (11). According to Eqs. (9) and (11), it is possible to reduce the phase change $\Delta \theta_{\mathrm{B}}$, by adequately choosing the powersplitting ratios $\rho_{i}$ (see Fig. 3). However, when $\Delta \theta_{\mathrm{B}}<\pi$, the MZI does not present exactly the required $n \times \pi$ phase discontinuity, but a maximum phase change $\Delta \theta_{\mathrm{B}}$ in a bandwidth given by:

$$
\Delta \nu_{\theta}=\frac{\arccos (\chi)}{\tau \pi}
$$

Furthermore, as $\chi$ is necessarily lower than unity, the amplitude response does not decay to zero, as required. By these reasons, the fractional differentiation operation of a MZI is more approximate than in the integer case. Fig. 4A and B shows the amplitude and phase response, respectively, for the bar port transfer function $B(\nu)$, as compared with the ideal transfer function $(-j \omega)^{n}$, with $n=0.7$ and delay time $\tau=10$ ps (i.e., $100 \mathrm{GHz}$ of separation between channels in a wavelength division multiplexer application). In this case, the required phase change is $\Delta \theta_{\mathrm{B}}=0.7 \times \pi$, which implies $\chi \cong 0.891$ (see Eq. 11). It can be observed that there is a reasonable degree of resemblance between both transfer functions (ideal and proposed), provided the band of interest had a spectral extension below $\tau^{-1}$, centered at $\nu_{\mathrm{B}}$, i.e., $|\nu|<50 \mathrm{GHz}$ in this example.

Continuing with the interferometric approach to photonic differentiation, we should mention here the previous work of Park et al. (2007). They 
A

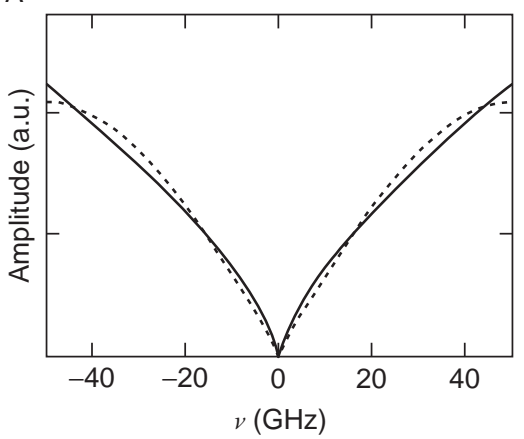

B

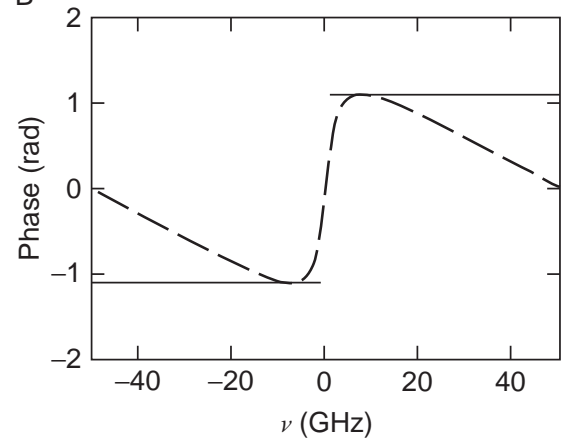

Fig. 4 Ideal (solid line) and proposed (dashed line) 0.7th-order differentiator frequency response in amplitude (A) and phase (B), in baseband frequency. From CuadradoLaborde, C. (2008). All-optical ultrafast fractional differentiator. Optical and Quantum Electronics, 40, 983-990. https://doi.org/10.1007/s11082-009-9282-5.

experimentally demonstrated a first-order photonic differentiator by using a Michelson-type interferometer and a free-space bulk-optics approach. Soon after, Park, Ahn, and Azaña (2008) presented a fiber-optic interferometerbased first-order differentiator with a feedback control loop for achieving stable operation. However, it was Zheng, Yang, et al. (2014), who first experimentally demonstrates the viability of Cuadrado-Laborde (2008), outlined above. Their device employed on-chip complementary metaloxide semiconductor (CMOS) technology. Further, by applying different voltages to both arms of the MZI they controlled the coupling ratios, which in turn modify the parameters $\chi$ and $\eta$, see Eqs. (9) and (10). Therefore, they could tune the fractional differentiation order $n$ through the change of $\Delta \theta_{\mathrm{B}}$ according to Eq. (11), see also Fig. 3. Fig. 5 shows the measured transfer function (in intensity) of the MZI, under broadband illumination. The deepest notch at the optical wavelength of $\sim 1552.6 \mathrm{~nm}$ represents the integer-order $(n=1)$ operation point. In this case, and due to the natural imperfections of the device, it is necessary to apply a nonzero voltage (exactly $-1.2 \mathrm{~V}$ ) to equalize both power-splitting ratios. However, nothing limits the possibility to improve the fabrication performance to reach an integer-order behavior at zero applied voltage.

Interestingly, Zheng, Yang, et al. (2014) also characterized dynamically their device, with the experimental setup shown in Fig. 6. This setup can be easily divided horizontally at the middle, defining the upper and lower section. In the upper section, there was all the necessary equipment to generate wavelength-tunable short transform-limited light pulses; whereas in the 


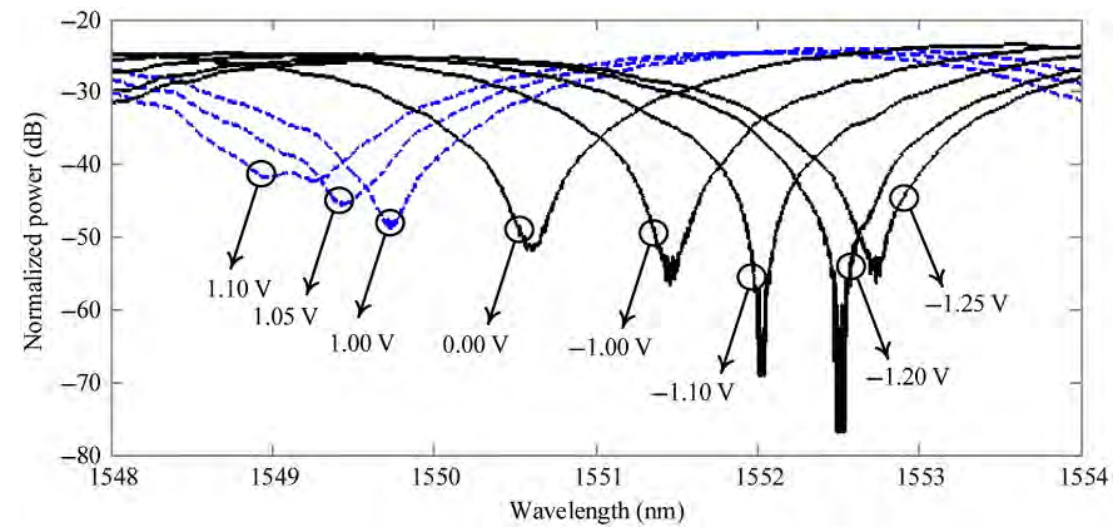

Fig. 5 Measured transfer functions of the $\mathrm{MZI}$ at different voltages. From Zheng, A., Yang, T., Xiao, X., Yang, Q., Zhang, X., \& Dong, J. (2014). Tunable fractional-order differentiator using an electrically tuned silicon-on-isolator Mach-Zehnder interferometer. Optics Express, 22, 18232-18237. https://doi.org/10.1364/OE.22.018232.

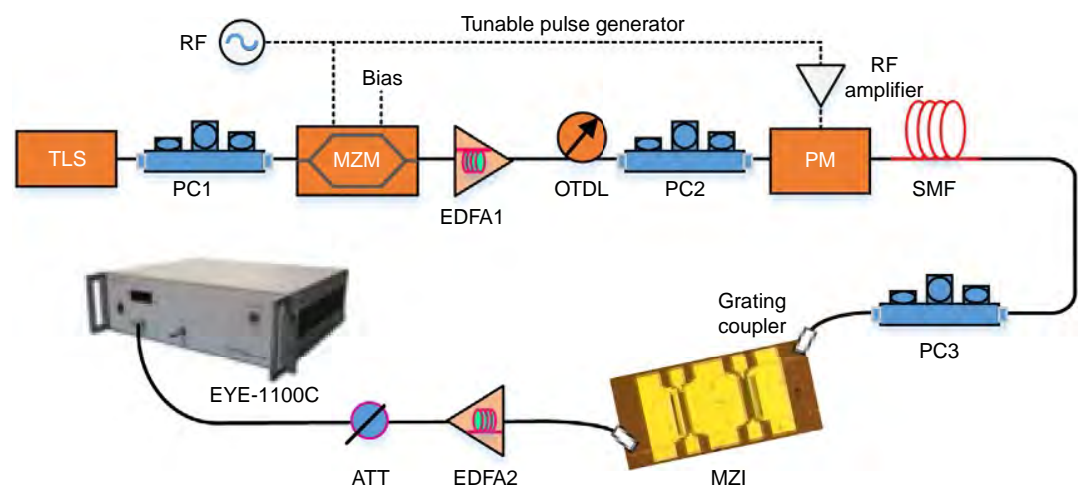

Fig. 6 Experimental setup for PFD with on-chip MZI structure. TLS: tunable laser source; $P C$ : polarization controller; MZM: Mach-Zehnder modulator; EDFA, OTDL: an optical tunable delay line; RF: radio frequency; $P M$ : phase modulator; and SMF: single mode fiber. From Zheng, A., Yang, T., Xiao, X., Yang, Q., Zhang, X., \& Dong, J. (2014). Tunable fractional-order differentiator using an electrically tuned silicon-on-isolator Mach-Zehnder interferometer. Optics Express, 22, 18232-18237. https://doi.org/10.1364/OE.22.018232.

lower section, it was the on-chip CMOS PFD (the voltage source used to tune the fractional differentiator order was not shown). Additionally, and given the high insertion and transmission losses typical of these devices $(-24 \mathrm{~dB}$ in this specific case), the output signal of the MZI chip was amplified by an erbium-doped fiber amplifier (EDFA) and next analyzed through a high-speed oscilloscope. The tunable pulse generator generates 

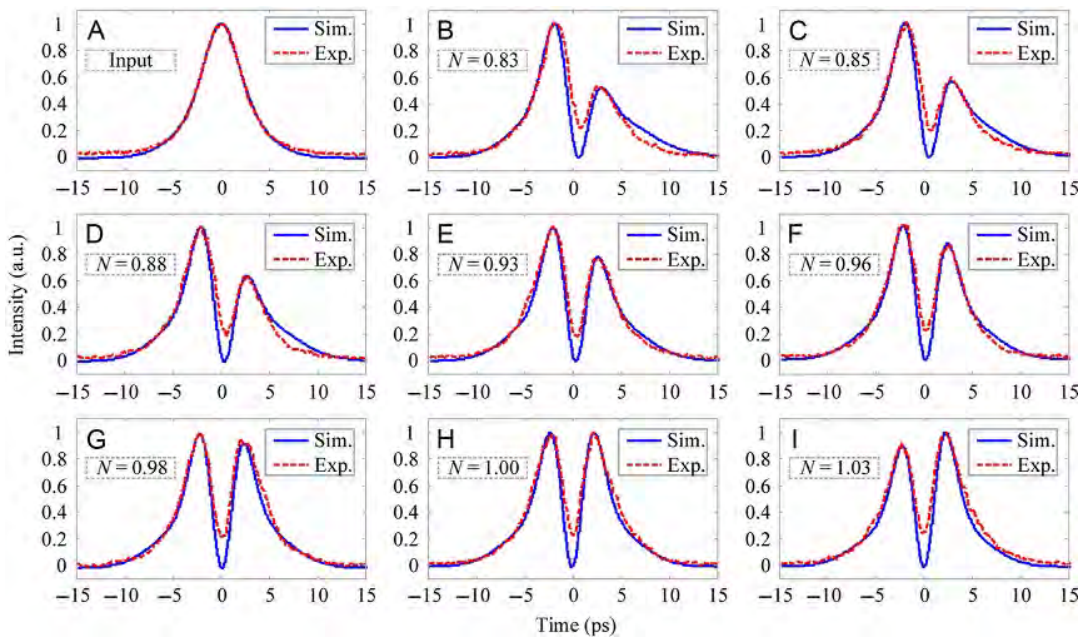

Fig. 7 (A) An input Gaussian pulse with an FWHM of 5.4 ps, and the differentiated pulses at the different voltages corresponding to differentiation orders of (B) $n=0.83$, (C) $n=0.85$, (D) $n=0.88$, (E) $n=0.93$, (F) $n=0.96$, (G) $n=0.98$, (H) $n=1.00$, and (I) $n=1.03$. From Zheng, A., Yang, T., Xiao, X., Yang, Q., Zhang, X., \& Dong, J. (2014). Tunable fractional-order differentiator using an electrically tuned silicon-on-isolator Mach-Zehnder interferometer. Optics Express, 22, 18232-18237. https://doi.org/10.1364/OE.22.018232.

a Gaussian-like pulse train with a time width of 5.4 ps (see Fig. 7). When the applied voltages changed from 1.10 to $-1.25 \mathrm{~V}$ on the MZI, and after fine wavelength matching between the tunable laser source and the notch of the PFD, the output differentiated waveforms were registered by the oscilloscope (see Fig. 7B-I). Simultaneously, the authors also simulated numerically the expected waveforms for fractional-order differentiation with orders $n=0.83,0.85,0.88,0.93,0.96,0.98,1.00$, and 1.03 , respectively. One can see there is a reasonable agreement between the measured light pulses and the simulated waveforms. Finally, it is worth mentioning that a photonic differentiator, regardless of being fractional or integer, it is a highly energetically inefficient device; due to the high-pass behavior of the filtering involved in the process. This fact, combined with the high insertion loss (typical of silicon on-chip solutions), precludes the direct detection of the fractional differentiated pulses by typical detectors in this kind of setups. We will see in the following that with in-fiber solutions the use of an EDFA stage at the output is not essential.

The first in-fiber experimental MZI approach to PFD came from Poveda-Wong et al. (2016a). In turn, this could be thought of as a 
generalization of the integer-order LPG-based interferometer previously developed by Slavík, Park, Krčmař́k, and Azaña (2009). As we shown above (Cuadrado-Laborde, 2008), an asymmetrical and unbalanced MZI have a spectral transfer function which approximately resembles the filter function required to perform a fractional-order differentiation, see Eq. (6). The setup proposed by Poveda-Wong et al. (2016a) is a modal MZI defined by a pair of long-period gratings (LPGs) written sequentially in the same piece of a stripped optical fiber (see Fig. 8). When compared with a direct implementation of a MZI structure with an all-fiber configuration (i.e., with two fiber couplers), this approach has the key advantage of an increased stability vs environmental fluctuations; because both arms of the interferometer are simultaneously exposed to nearly identical environmental variations. Succinctly explained, the LPGs establish a coaxial MZI for fiber modes propagating through the core and cladding in the same direction.

An asymmetrical and unbalanced MZI-i.e., splitting/coupling ratio different from $1 / 2$ and different path lengths — has a spectral transfer function that resembles the requirements for fractional-order differentiation (CuadradoLaborde, 2008). It has four ports: two input ports and two output ports. The output ports are usually named bar and cross ports, depending on if most of the light appears in the same waveguide of the input or not, respectively. In an LPG MZI, the inputs and outputs are two fiber modes at the beginning and end of the optical fiber section in which the device is constructed (see Fig. 8); thus, the arms of the interferometer are typically the $\mathrm{LP}_{01}$ core mode and the $\mathrm{LP}_{0 m}$ cladding mode excited by the LPG (see Fig. 8). In the following, it is assumed that only the core guided mode is initially excited by the pulse launched at the input of the LPG MZI. The first LPG couples a certain amount of power from the core to the cladding, characterized by $\rho_{1}$, with $0 \leq \rho_{1} \leq 1$. After light propagates in both arms, the second LPG couples back to the core a

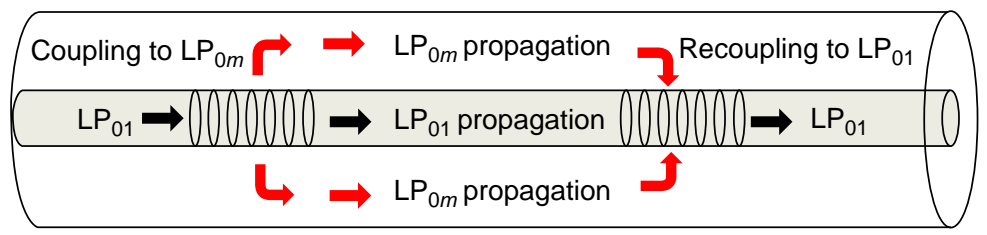

Fig. 8 Scheme of the proposed fractional differentiator based on the use of a LPG MZI. From Poveda-Wong, L., Carrascosa, A., Cuadrado-Laborde, C., Cruz, J. L., Díez, A., \& Andrés, M. V. (2016a). Experimental demonstration of fractional order differentiation using a longperiod grating-based in-fiber modal interferometer. Optics Communications, 380, 35-40, https://doi.org/10.1016/j.optcom.2016.05.083. 
certain fraction of power from the cladding, characterized by $\rho_{2}$, with $0 \leq \rho_{2} \leq 1$; where it is finally coherently recombined with the light propagated by the core, not coupled to the cladding by the first LPG. In an LPG MZI both arms have the same length; it is the difference between the core and claddingmode propagation constants $\beta_{i}$ which induces a time delay $\tau$. From Eqs. (9) and (11), it is possible to connect the fractional differentiation order $n$ with the power-splitting ratios $\rho_{1}$ and $\rho_{2}$ by using $\Delta \theta_{\mathrm{B}}=n \times \pi$. However, this solution is not unique; for this reason, by arbitrary fixing the power-splitting ratio of the first LPG $\left(\rho_{1}=1 / 2\right)$, the power-splitting ratio of the second LPG $\left(\rho_{2}\right)$ can be expressed as a function of the fractional differentiator order $n$ (PovedaWong et al., 2016a):

$$
\rho_{2}=\frac{1}{2}+\frac{1}{2}\left[2 \tan ^{2}\left(\frac{\pi n}{2}\right)+1\right]^{-1}
$$

According to Eq. (13), it is possible to obtain any arbitrary fractional differentiator order $n$, just adequately choosing the second power-splitting ratio $\rho_{2}$, whenever $\rho_{1}=1 / 2$. However, it is worth noting that the MZI does not present exactly the required $n \times \pi$ phase discontinuity, but a maximum phase change $\Delta \theta$ in a bandwidth $\Delta \nu_{\theta}$ (see Eq. 12). In other words, the useful spectral bandwidth - which is restrained between $\Delta \nu_{\theta}$ and the maximum operation bandwidth-increases as the fractional differentiator order approaches one. In the other limit for $n \rightarrow 0$, the bandwidth of the phase transition approaches half the full operative bandwidth (Poveda-Wong et al., 2016a). In conclusion, for an optimal performance, the bandwidth of the input optical signal should be higher than the phase transition bandwidth $\Delta \nu_{\theta}$, but lower than the maximum operative bandwidth.

In Poveda-Wong et al.'s (2016a) approach to PFD, the LPG MZI was constructed by recording two different LPGs in a single piece of a borondoped photosensitive fiber (PS980 by Fibercore, numerical aperture of 0.13 and cut-off wavelength of $980 \mathrm{~nm}$ ), by using the point-by-point technique. The selected periodicity for both LPGs was of $189.7 \mu \mathrm{m}$. In the first LPG the number of periods was 28 , resulting in a total length of $5.3 \mathrm{~mm}$. The transmission response for $\mathrm{LPG}_{1}$ was followed during the fabrication process by measuring the transmitted light provided by a led source in an optical spectrum analyzer (spectral resolution $20 \mathrm{pm}$ ). The recording of $L P \mathrm{G}_{1}$ was interrupted when the transmission reached a resonance depth of $-3 \mathrm{~dB}$ (i.e., $\left.\rho_{1}=0.5\right)$ at $1039.8 \mathrm{~nm}$. Next, $\mathrm{LPG}_{2}$ was recorded slightly shorter; i.e., 25 periods, giving a $\mathrm{LPG}_{2}$ length of $4.7 \mathrm{~mm}$ (with the remaining recording parameters unchanged, as compared with $\left.\mathrm{LPG}_{1}\right)$. As a consequence of this, 
the estimated transmittance dip for $\mathrm{LPG}_{2}$ was of $-1.8 \mathrm{~dB}$, i.e., $\rho_{2}=0.66$. Following Eq. (13), this corresponds to a fractional differentiator order of $n=0.5$. On the other hand, the separation between LPGs is determined by the required operation bandwidth, which in turn is fixed by the spectral characteristics of the input optical signal to be processed (Lee \& Nishii, 1999), which should be known a priori. In this case, the optimum processing bandwidth was achieved, by selecting a distance between LPGs of $65 \mathrm{~mm}$ (centerto-center distance). Fig. 9 shows the observed interference pattern in the optical spectrum analyzer (in transmission), when the LPG MZI is illuminated by a led source. The central peak of transmission minimum is located at $1039.8 \mathrm{~nm}$; whereas the operation bandwidth—which is given approximately by the separation between the transmission maxima at each side of the central transmission minimum-is around $3 \mathrm{~nm}$ (see Fig. 9). This value determined a maximum operative bandwidth of $83 \mathrm{GHz}$.

The LPG-based MZI was dynamically tested by differentiating the light pulses provided by a passively mode-locked ytterbium fiber laser; which can be approximately fitted with an hyperbolic secant profile $f(t)=\operatorname{sech}\left(t / T_{0}\right)$, with $T_{0}=13$ ps; i.e., a FWHM of 23 ps (see Fig. 10A). Additionally, its phase profile was measured, resulting in $\exp \left(-j C t^{2} / 2 T_{0}^{2}\right)$, with $C=-30$.

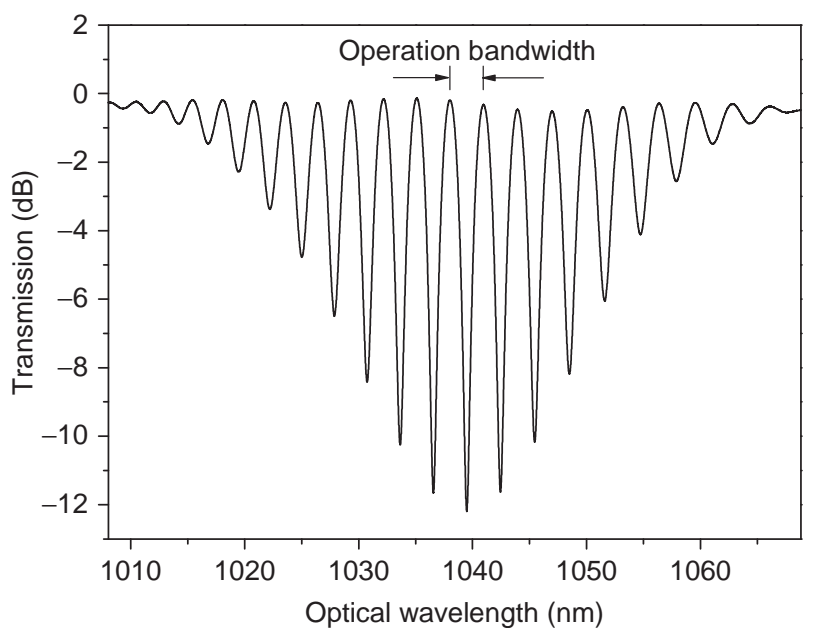

Fig. 9 Measured spectral power transmission of the LPG MZI. The device works as a 0.5 th-order fractional differentiator in the central spectral region. From Poveda-Wong, $L$., Carrascosa, A., Cuadrado-Laborde, C., Cruz, J. L., Díez, A., \& Andrés, M. V. (2016a). Experimental demonstration of fractional order differentiation using a long-period grating-based in-fiber modal interferometer. Optics Communications, 380, 35-40, https://doi.org/10. 1016/j.optcom.2016.05.083. 
A

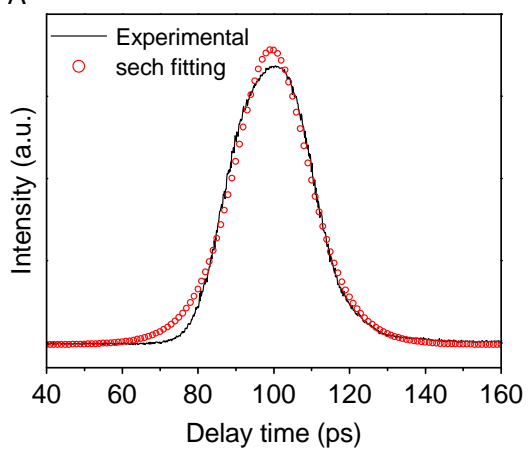

B

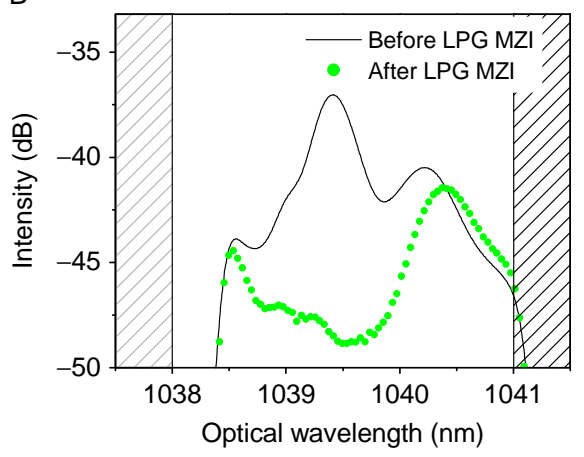

C

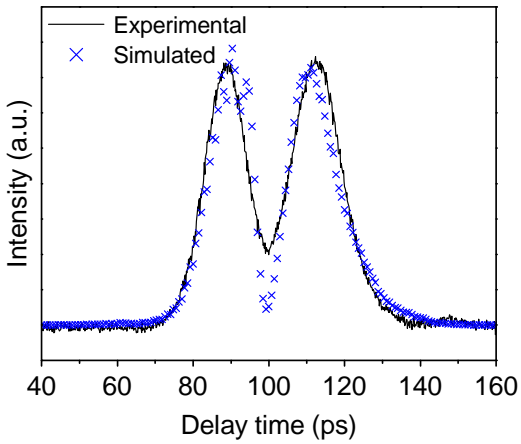

Fig. 10 (A) Temporal intensity profile at the input of the LPG MZI, together with its corresponding fitting. (B) Spectra of the light pulse before and after the LPG MZI. (C) Temporal intensity profile at the output of the LPG MZI, together with the simulated response of a 0.5 th-order fractional differentiator. From Poveda-Wong, L., Carrascosa, A., Cuadrado-Laborde, C., Cruz, J. L., Díez, A., \& Andrés, M. V. (2016a). Experimental demonstration of fractional order differentiation using a long-period grating-based in-fiber modal interferometer. Optics Communications, 380, 35-40, https://doi.org/10.1016/j.optcom. 2016.05.083.

Fig. 10B shows the spectra of the light pulses at the input and output of the LPG MZI. It can be observed that the input signal carrier is well aligned with the central resonance optical wavelength of the LPG MZI; as a result, the optical carrier is deeply suppressed in the output light pulse by more than $12 \mathrm{~dB}$ (see Fig. 10B). Fig. 10C shows the measured temporal intensity profile at the output of the LPG MZI. In the same figure, the simulated response of an ideal 0.5th-order fractional differentiator is also shown for comparison. There is a reasonably degree of resemblance between both experimental and simulated temporal profiles. 
We conclude the MZI approach to PFD by describing the very recent work of Liu, Zhang, and Yao (2017). They designed, constructed, and tested two different MZI-based proposals for PFD. The first proposal (named by the authors as passive tuning) employed a multimode interference (MMI) coupler as one of the two 3-dB couplers of the MZI (see Fig. 11D). In this case, it is clear from Eqs. (9) and (11), that by changing the polarization state of the input optical signal, the coupling coefficient $\rho_{i}$ of the MMI is changed, which leads to the change of the phase shift in the destructive interference wavelength according to Eq. (11). Therefore, the control in the polarization state of the signal under test is used as a tuning of the fractional differentiator order. Their second proposal (named by the authors as active tuning) employed two cascaded MZIs, where only one of them is balanced (i.e., a MZI with both power-splitting ratios equal to $3 \mathrm{~dB}$ ) (see Fig. 11E). Additionally, there are two different phase modulators (PMs), where each one is incorporated in one of the two arms of each MZI. The balanced MZI acts as an active tunable coupler, which is effectively used to actively tune the fractional order of photonic differentiation; whereas the PM in the unbalanced

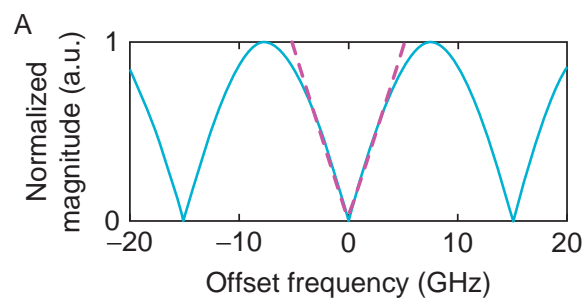

C
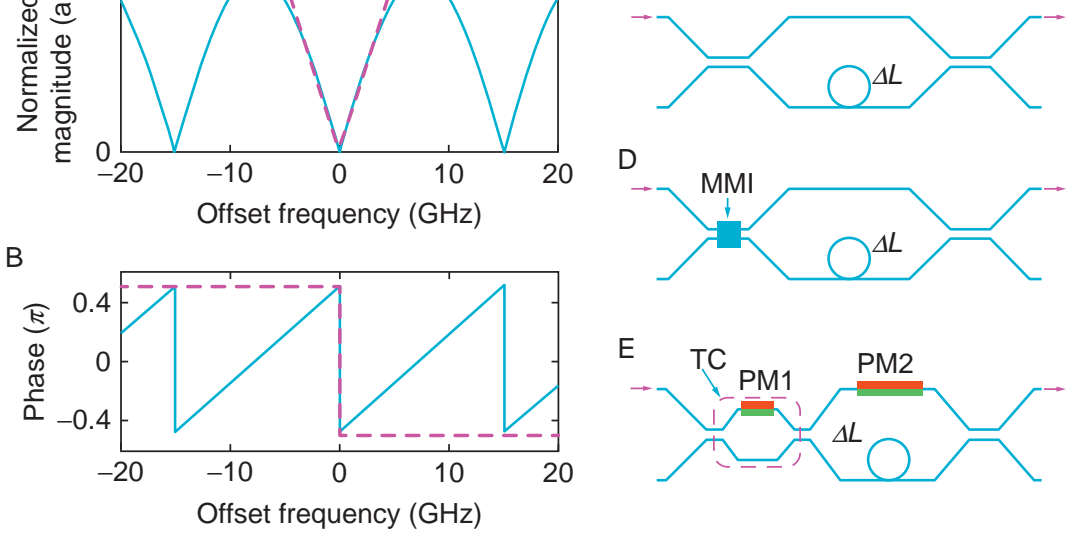

Fig. 11 (A) The magnitude and (B) phase responses of an MZI (solid line) and an ideal photonic temporal differentiator (dashed line). (C) An MZI with a length difference $\Delta L$ between the two arms. (D) The passive and (E) active design concept of a tunable fractional photonic temporal differentiator based on an SOI MZI. MMI: multimode interference; PM: phase modulator; and TC: tunable coupler. From Liu, W., Zhang, W., \& Yao, J. (2017). Silicon-based integrated tunable fractional order photonic temporal differentiators. Journal of Lightwave Technology, 35, 2487-2493. https://doi.org/10.1109/JLT.2017. 2688468. 
MZI is used to tune the operating wavelength. Both proposals were designed and fabricated in a CMOS compatible silicon-on-isolator (SOI) platform, further details about the fabrication details can be found in the original publication of Liu et al. (2017). Fig. 11A and B shows the typical MZI response in amplitude and phase, respectively, as compared with the ideal fractional differentiation (see Eq. 6); whereas Fig. 11C-E shows schematically the standard MZI, the passive tuning proposal, and the active tuning proposal, respectively.

Continuing with Liu et al.'s (2017) work, we will first show the main results in their passive MZI proposal (see Fig. 11D). The spectral response shown in Fig. 12A was measured with an optical vector analyzer; the measured operative bandwidth was $0.74 \mathrm{~nm}$ (or $92.4 \mathrm{GHz}$ ). By changing the polarization state of the input optical signal, the spectral response and phase shift at the destructive-interference wavelength are changed, as shown in Fig. 12B and C. This is also confirmed in their simulations, as shown in Fig. 12D. This demonstrates that the polarization state of the input optical signal can be used to tune the fractional differentiator order $n$.

An optical Gaussian pulse train with a pulse width of 11.8 ps (the spectral characteristics were not given by the authors, but we assume are transform limited) was sent to the passive PFD through a polarization controller (PC) (see Fig. 13A). By controlling the polarization state of the input Gaussian pulse, a temporal differentiated pulse with a tunable fractional order was obtained. Fig. 13B-D shows the differentiated pulse with a fractional order of $0.55,0.85$, and 1 , respectively; together with the simulation results of an ideal differentiation according to Eq. (6) (dashed lines).

Liu et al. (2017) also characterize the behavior of their active proposal. The spectral responses of the active differentiator while applying a voltage of $-1,-2$, and $-3 \mathrm{~V}$ to the PM in the second MZI (PM2), as shown in Fig. 11E, is plotted in Fig. 14A. In this case, the measured operative bandwidth was almost twice as compared with the preceding passive approach $(1.37 \mathrm{~nm}$, or $171.1 \mathrm{GHz})$. Additionally, it should be noted that the spectral notch shifts when the fractional order is tuned. By changing the voltage applied to the PM in the first MZI (PM1), the spectral response and phase shift at the destructive interference wavelength are changed, as shown in Fig. 14B and C, where the spectral shifting was corrected for comparison purposes. The voltages applied to PM1 are $0,1,2,3.5$, and $5 \mathrm{~V}$, corresponding to the fractional differentiations orders $n=0,0.16,0.41,0.60$, and 1 , respectively. 

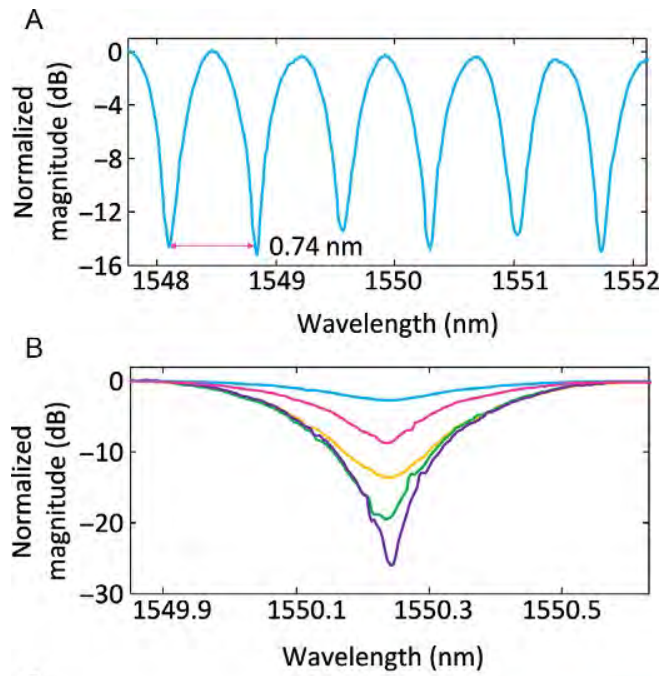

C
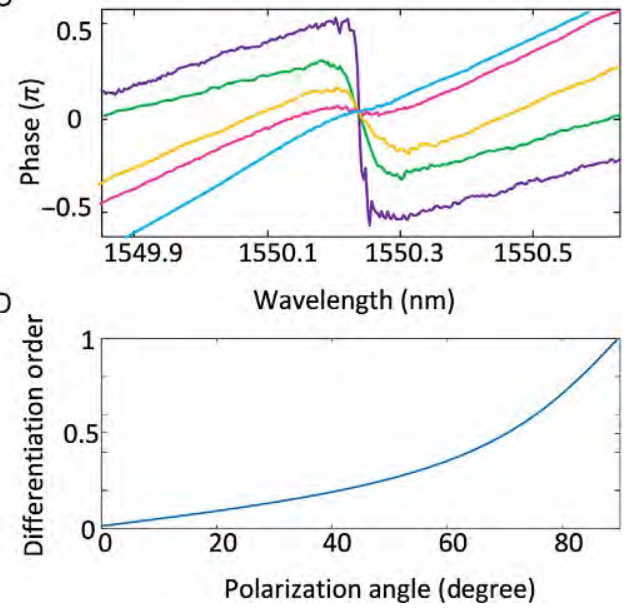

Fig. 12 Experimental results of the passive photonic temporal differentiator. (A) The spectral response of the MZI photonic temporal differentiator. (B) The spectral response and (C) phase response of the differentiator when tuning the polarization state of the input optical signal. (D) Simulation results to show the differentiation order as a function of the state of polarization of the input optical light. From Liu, W., Zhang, W., \& Yao, J. (2017). Silicon-based integrated tunable fractional order photonic temporal differentiators. Journal of Lightwave Technology, 35, 2487-2493. https://doi.org/10.1109/JLT.2017.2688468.

Finally, in Fig. 15, the dynamical characterization of the behavior of the proposal of Liu et al. (2017) is presented. An optical Gaussian pulse with a pulse width of $21.9 \mathrm{ps}$ (unfortunately, the spectral characteristics were not given by the authors, but we assume this pulse is transform limited) was sent 


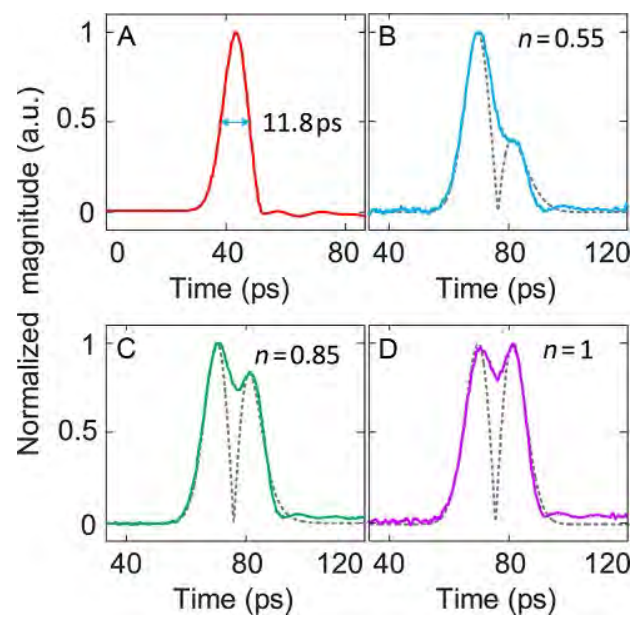

Fig. 13 Experimental results of the passive PFD. (A) The input Gaussian pulse with a temporal width of $11.8 \mathrm{ps}$. The measured (solid) and simulated (dashed) fractional differentiation of the input Gaussian pulse with a fractional order $n=0.55$ (B), 0.85 (C), and 1 (D). From Liu, W., Zhang, W., \& Yao, J. (2017). Silicon-based integrated tunable fractional order photonic temporal differentiators. Journal of Lightwave Technology, 35, 2487-2493. https://doi.org/10.1109/JLT.2017.2688468.

to the active device through a PC. By changing the voltage applied to PM1, temporally differentiated pulses with a fractional order of $0.45,0.70$, and 1 were obtained, as shown in Fig. 15B-D, respectively. Simulation results of an ideal photonic temporal differentiator are also given in Fig. 15B-D (dashed lines). As can be seen, the experimental results have a good agreement with the simulated results except some small difference in notch depth. Of course, at every differentiator order, the laser source was tuned to the notch of the PFD. Although it is true that this device provides a fast tunability reconfiguration characteristic, it does to different wavelengths. In certain applications this could be a handicap.

\subsubsection{The Bragg Grating Approach}

Chronologically, the second proposal for PFD came from CuadradoLaborde and Andrés (2009). They discussed the possibility to perform PFD with an asymmetrical $\pi$ phase shifted fiber Bragg grating (APS-FBG) operated in reflection. Fig. 16 shows the proposed in-fiber fractional differentiator. Essentially, it is composed of two uniform period FBG separated by a $\pi$ phase shift, operated in reflection. 

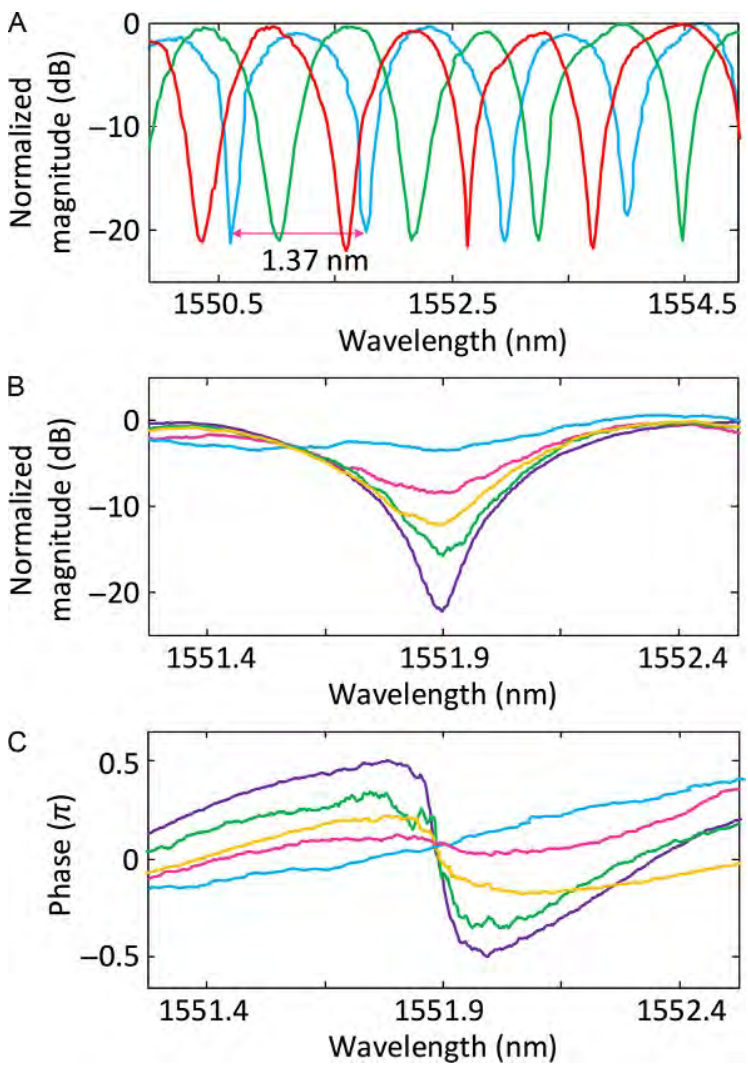

Fig. 14 Experimental results of the active photonic temporal differentiator. (A) The spectral response of the MZI photonic temporal differentiator when applying three different voltages to the PM in the second MZI. (B) The spectral response and (C) phase response of the differentiator when the voltage to the PM in the first $\mathrm{MZI}$ is tuned at five different values. From Liu, W., Zhang, W., \& Yao, J. (2017). Silicon-based integrated tunable fractional order photonic temporal differentiators. Journal of Lightwave Technology, 35, 2487-2493. https://doi.org/10.1109/JLT.2017.2688468.

The authors demonstrated that there are two different, but equivalent, ways to obtain the required spectral response: either by using the same index modulation depth $\Delta n_{1}=\Delta n_{2}$, but different gratings lengths at both sides of the $\pi$ phase shift, $L_{1} \neq L_{2}$, or vice versa; i.e., by using different index modulation depths $\Delta n_{1} \neq \Delta n_{2}$, but the same grating length at both sides of the $\pi$ phase shift, $L_{1}=L_{2}$. The final grating structure of the APS-FBG can be described by a $2 \times 2$ transfer matrix (Berger et al., 2007):

$$
\mathbf{T}_{\mathrm{APS}-\mathrm{FBG}}={ }^{1} \mathbf{T}_{\mathrm{FBG}} \mathbf{T}_{\mathrm{PS}}{ }^{2} \mathbf{T}_{\mathrm{FBG}},
$$




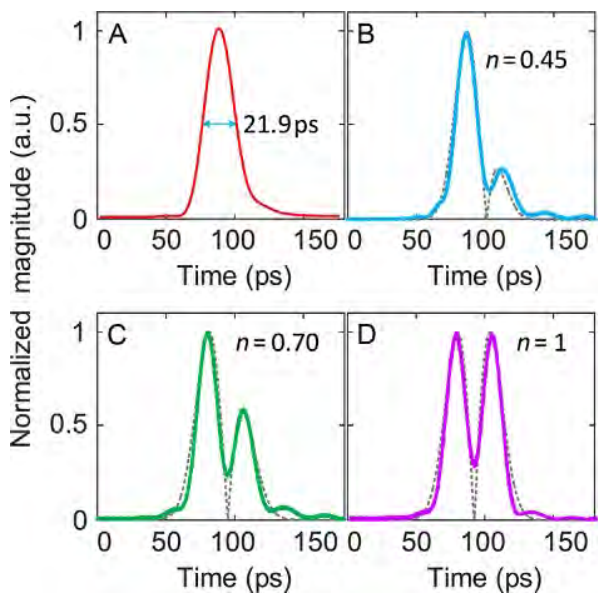

Fig. 15 Experimental results of the active photonic temporal differentiator. (A) The input Gaussian pulse with a temporal width of $21.9 \mathrm{ps}$. The measured (solid) and simulated (dashed) fractional differentiator of the input Gaussian pulse with a fractional order of (B) 0.45, (C) 0.70, and (D) 1. From Liu, W., Zhang, W., \& Yao, J. (2017). Silicon-based integrated tunable fractional order photonic temporal differentiators. Journal of Lightwave Technology, 35, 2487-2493. https://doi.org/10.1109/JLT.2017.2688468.

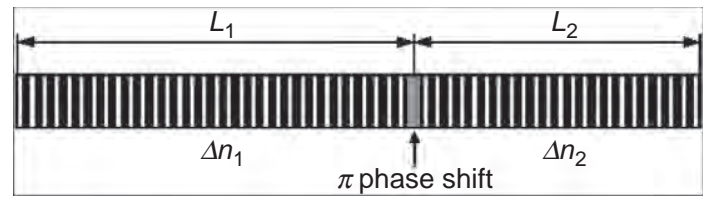

Fig. 16 Scheme of a FBG to perform a photonic fractional order differentiation. $\Delta n_{1}$ and $\Delta n_{2}$ are the index modulation depths, whereas $L_{1}$ and $L_{2}$ are the grating lengths at both sides of the $\pi$ phase shift, respectively. From Cuadrado-Laborde, C., \& Andrés, M. V. (2009). In-fiber all-optical fractional differentiator. Optics Letters, 34, 833-835. https://doi.org/10. 1364/OL.34.000833.

where $\mathbf{T}_{\mathrm{PS}}$ is a $2 \times 2$ diagonal matrix representing the phase shift $\varphi$ with elements $\exp ( \pm j \varphi / 2)$, and ${ }^{i} \mathbf{T}_{\mathrm{FBG}}$ is a $2 \times 2$ matrix representing each uniform FBG at both sides of the $\pi$ phase shift, where $i=1,2$ identifies the uniform FBG to the left and right of the phase shift, respectively. Its elements are (Yamada \& Sakuda, 1987):

$$
\begin{gathered}
{ }^{i} T_{11}={ }^{i} T_{22}^{*}=\left[\cosh \left(\gamma_{i} L_{i}\right)+j\left(\Delta \beta / \gamma_{i}\right) \sinh \left(\gamma_{i} L_{i}\right)\right] \exp \left(j \beta_{\mathrm{B}} L_{i}\right), \\
{ }^{i} T_{12}={ }^{i} T_{21}^{*}=-\left(\kappa_{i} / \gamma_{i}\right) \sinh \left(\gamma_{i} L_{i}\right) \exp \left[-j \beta_{\mathrm{B}} L_{i}\right],
\end{gathered}
$$


where ${ }^{*}$ denotes complex conjugation, with $\gamma_{i}{ }^{2}=\kappa_{i}{ }^{2}-\Delta \beta^{2}, \kappa_{i}=\pi \Delta n_{i} \lambda_{\mathrm{B}}{ }^{-1}$ is the coupling coefficient, $\Delta \beta=2 \pi n_{\text {eff }}\left(\lambda^{-1}-\lambda_{\mathrm{B}}{ }^{-1}\right)$ is the wavenumber detuning from the Bragg wavelength $\lambda_{\mathrm{B}}, n_{\mathrm{eff}}$ is the modal effective index, $\beta_{\mathrm{B}}$ is the propagation constant at the Bragg wavelength, and the length of each uniform FBG is $L_{i}=N_{i} \Lambda$, which was fixed for simplicity to be an integer number of the grating period $\Lambda=\lambda_{\mathrm{B}}\left(2 n_{\mathrm{eff}}\right)^{-1}$. The parameters $\lambda_{\mathrm{B}}$ and $n_{\text {eff }}$ are assumed to be the same for the left and right FBG. At $\nu=0$, the presence of the $\pi$ phase shift induces a reflection dip in the grating spectral reflection band, $\left|r_{\text {APS-FBG }}\right|$, whereas the phase $\theta_{\text {APS-FBG }}$ presents a maximum change $\Delta \theta$ in a narrow spectral bandwidth. In the single-reflection approximation, the final reflectivity can be expressed as:

$$
r_{\mathrm{APS}-\mathrm{FBG}} \approx r_{1}+r_{2}\left(t_{1}\right)^{2} \exp [j \varphi]
$$

where $r_{i}={ }^{i} T_{21} /{ }^{i} T_{11}$ is the field reflectivity of the $i$ th FBG, and $t_{1}=\left({ }^{i} T_{11}\right)^{-1}$ is the field transmissivity of the first FBG. By using Eq. (17), it is possible to express the APS-FBG phase reflectivity as

$$
\theta_{\mathrm{APS}-\mathrm{FBG}}(\Delta \beta) \approx-\Delta \beta L_{1}+\operatorname{atan}\left[\frac{\varepsilon_{\kappa} \varepsilon_{L} \sin \left(\Delta \beta L_{T}\right)}{1-\varepsilon_{\kappa} \varepsilon_{L} \cos \left(\Delta \beta L_{T}\right)}\right]
$$

where $L_{T}=L_{1}+L_{2}, \varepsilon_{\kappa}=\kappa_{2} / \kappa_{1}$, and $\varepsilon_{L}=L_{2} / L_{1}$. The maximum phase change $\Delta \theta$ can be obtained by finding the roots of the first derivative of Eq. (18), which are given by:

$$
\Delta \beta_{0}=\frac{1}{L_{T}} \operatorname{acos}\left(\frac{1+\varepsilon_{L}^{2} \varepsilon_{\kappa}^{2}\left(2+\varepsilon_{L}\right)}{\varepsilon_{\kappa} \varepsilon_{L}\left(3+\varepsilon_{L}\right)}\right)
$$

In this way, by replacing Eq. (19) in Eq. (18), we obtain $\Delta \theta / 2$, and taking into account that $\Delta \theta=n \times \pi$, the authors arrived at an analytical expression for $n$ as a function of the FBG parameters:

$$
\left.n \approx \frac{2}{\pi} \theta_{\mathrm{APS}-\mathrm{FBG}}(\Delta \beta)\right|_{\Delta \beta=\Delta \beta_{0}}
$$

with $\theta_{\text {APS-FBG }}$ given in Eq. (18). Fig. 17A shows the dependence of the fractional order of differentiation $n$ with the relative change of the grating length $\varepsilon_{L}$, by keeping $N_{1}=1800$ fixed and varying $N_{2}$, when $\lambda_{\mathrm{B}}=1550 \mathrm{~nm}, n_{\mathrm{eff}}=1.46$, and $\Delta n_{1}=\Delta n_{2}=4 \times 10^{-4}$, for both the numerical (exact) solution and the approximate (analytical) solution given in Eq. (18). In a similar way, Fig. 17B shows the dependence of $n$ with 
A

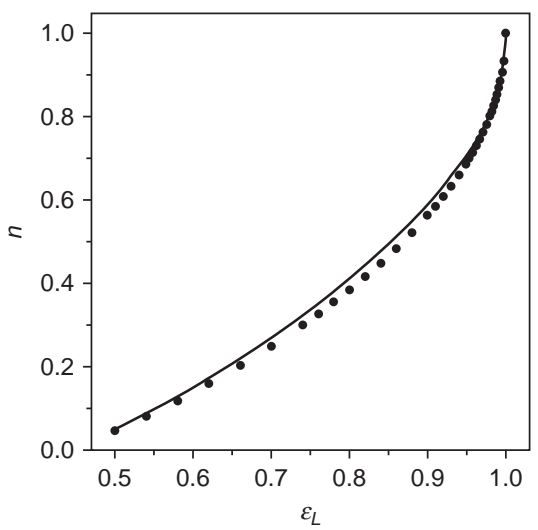

B

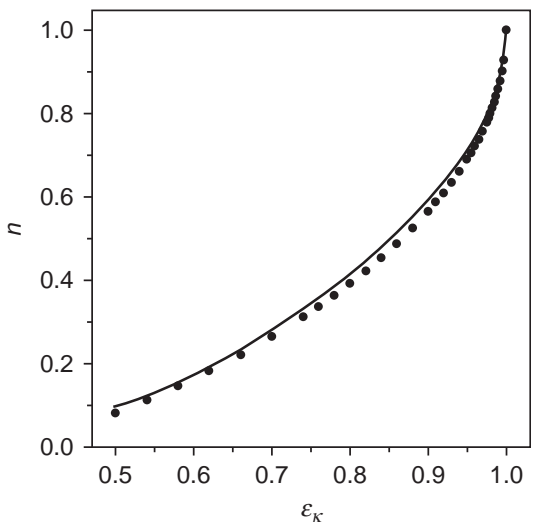

Fig. 17 Differentiator order $n$ as a function of the relative change of the grating lengths $\varepsilon_{L}(\mathrm{~A})$ and the index modulation depths $\varepsilon_{\kappa}(\mathrm{B})$. The scatter points represent the numerical (exact) solution, whereas the solid line represents the approximate (analytical) solution given in Eq. (7). From Cuadrado-Laborde, C., \& Andrés, M. V. (2009). In-fiber all-optical fractional differentiator. Optics Letters, 34, 833-835. https://doi.org/10.1364/OL.34.000833.

the relative change of the index modulation depth $\varepsilon_{\kappa}$, by keeping $\Delta n_{1}=4 \times 10^{-4}$ fixed and varying $\Delta n_{2}$, with $N_{1}=N_{2}=1800$, and same parameters as before. It should be noted that for practical purposes a given $n$ can be obtained by the same relative change of FBG length $\varepsilon_{L}$ or index modulation depth $\varepsilon_{\kappa}$. Fig. $18 \mathrm{~A}$ and B shows the field reflectivity $\left|r_{\mathrm{APS}-\mathrm{FBG}}\right|$ and reflection phase $\theta_{\mathrm{APS}-\mathrm{FBG}}$, respectively, as compared with the ideal transfer function $H(\nu)$ for $n=0.63$, i.e., the case $\varepsilon_{\kappa}=0.9275$ in Fig. 17B. We observe that there is a reasonable degree of resemblance between both transfer functions (ideal and proposed), provided the band of the signal matches adequately the central part of the transfer function. This band should be higher than the bandwidth of the phase change $\left(\Delta \nu_{\min } \approx \Delta \beta_{0} c / \pi n_{\text {eff }}\right)$, see the top part of Fig. $18 \mathrm{~B}$, but lower than the bandwidth fixed by the maxima of the APS-FBG reflectivity $\left(\Delta \nu_{\max } \approx c / L_{T} n_{\text {eff }}\right)$, see the top part of Fig. 18A.

Continuing with FBGs, Li, Shao, Albert, and Yao (2011) proposed the first tunable PFD (from a chronological point of view). It is known that tilted FBGs (TFBG) have multiple cladding-mode resonances, whose phase response is highly polarization dependent. Thus, they propose tuning the fractional order of the photonic differentiation through the polarization state of the optical input signal. Fig. 19 shows schematically the 

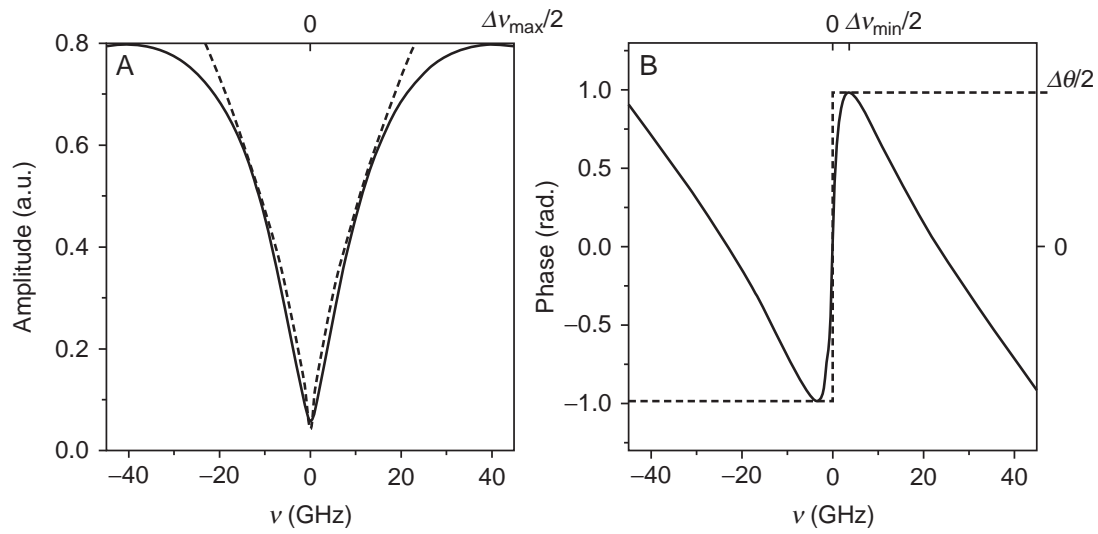

Fig. 18 Ideal (dashed curve) and proposed (solid curve) 0.63th-order differentiator frequency response in amplitude (A) and phase (B), in baseband frequency. From Cuadrado-Laborde, C., \& Andrés, M. V. (2009). In-fiber all-optical fractional differentiator. Optics Letters, 34, 833-835. https://doi.org/10.1364/OL.34.000833.
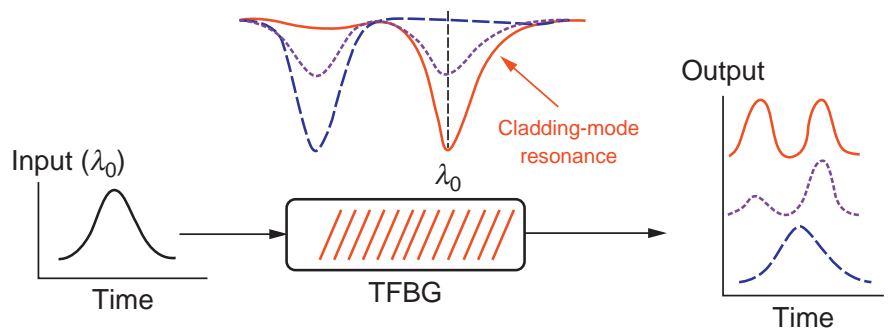

Fig. 19 Operation of a continuously tunable temporal photonic fractional differentiator based on a TFBG. When the incident light wave is adjusted with three different polarization states, a differentiated output with three different differentiation orders is achieved. From Li, M., Shao, L. Y., Albert, J., \& Yao, J. (2011). Continuously tunable photonic fractional temporal differentiator based on a tilted fiber Bragg grating. IEEE Photonics Technology Letters, 23, 251-253. https://doi.org/10.1109/LPT.2010.2098475.

behavior in transmission of a TFBG. The resonance at the longest wavelength corresponds to the core mode reflection; whereas at the shorter wavelength we have the resonances corresponding to the counterpropagating coupling between the core mode and the cladding modes. With a linearly polarized input light, the TFBG can couple to two distinct groups of cladding modes depending on the orientation of the light polarization that is parallel or perpendicular to the tilt plane. Tuning 
the polarization state between these extremes yields pairs of neighboring resonances with adjustable relative amplitudes. When a signal with an optical carrier located in one of the loss peaks, the pulse will be differentiated by the TFBG with a fractional order determined by the input polarization state.

Fig. 20A shows the transmission spectrum of a TFBG with a tilt angle of 4 degree. Fig. 20B shows the magnitude and phase response of one channel of the transmission spectrum. The phase shift generated at the resonance frequency is $2.54 \mathrm{rad}$, which translates in a fractional order of $n=2.54$ / $\pi \cong 0.81$. However, when translating this fractional order to the amplitude response of the fractional filter (see Eq. 6); i.e., $\left|\omega-\omega_{0}\right|^{0.81}$ there is a noticeable mismatch with the measured amplitude response. This became easily
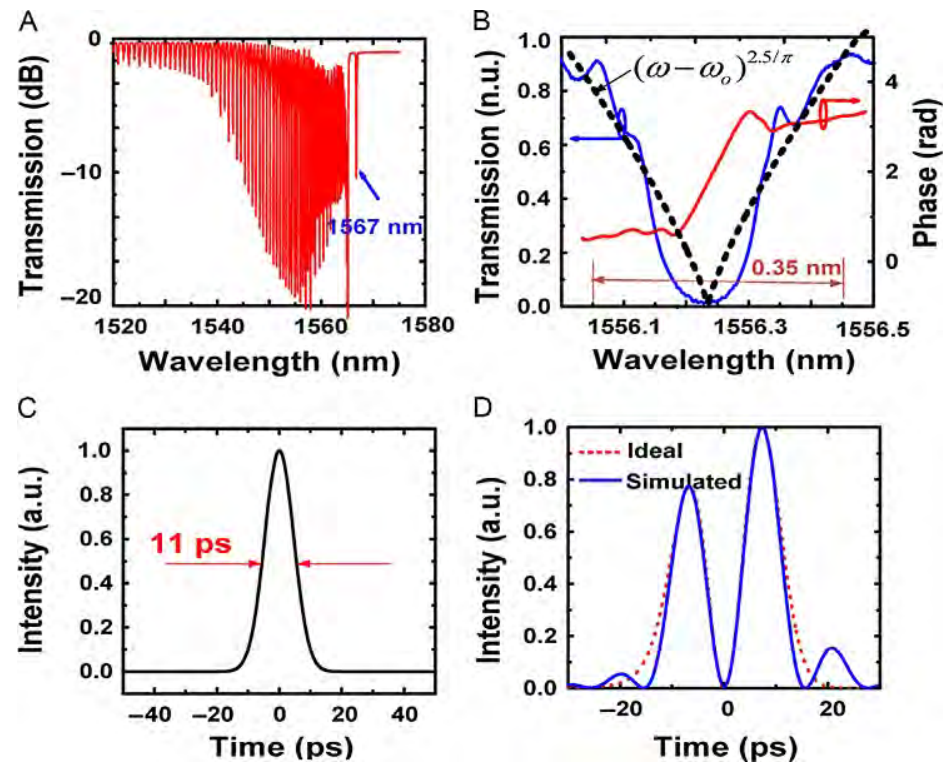

Fig. 20 (A) Transmission spectrum of a 4 degree TFBG. (B) Magnitude and phase responses of one channel of the transmission spectrum. The dotted line is a theoretical filter response in magnitude of a fractional differentiation filter with fractional order $n=2.54 / \pi \cong 0.81$. (C) An input Gaussian pulse with a pulse width of 11 ps. (D) Simulated output pulse from the 4 degree TFBG and an ideal photonic differentiator with a fractional order of $n=2.54 / \pi \cong 0.81$. From Li, M., Shao, L. Y., Albert, J., \& Yao, J. (2011). Continuously tunable photonic fractional temporal differentiator based on a tilted fiber Bragg grating. IEEE Photonics Technology Letters, 23, 251-253. https://doi.org/10. 1109/LPT.2010.2098475. 
revealed in Fig. 20B by comparing both amplitude traces: measured and simulated. However, this does not prevent an acceptable performance, according to these authors. As it can be observed by simulating the 0.81th-order fractional differentiation of an input Gaussian pulse (see Fig. 20C). Fig. 20D shows both, the theoretical 0.81th-order fractional differentiation and the simulated response to this pulse of the TFBG, by using the phase and amplitude measured.

The viability of this proposal to effectively fractional differentiate light pulses with a tuning fractional order $n$ was verified experimentally in $\mathrm{Li}$ et al. (2011). However, since a $\pi$ phase shift (corresponding to a fractional differentiator order $n=1$ ) cannot be achieved in the cladding-mode resonance by only using a single TFBG, in their experiment two different TFBG were cascaded. Thus, they were able to experimentally demonstrate tunability for PFD in the range $0.81 \leq n \leq 1.42$ for light pulses in the tens of gigahertz regime.

In the aforementioned work by $\mathrm{Li}$ et al. (2011), the tunability was reached by the strong polarization dependence of the phase change at the resonance wavelength. This proposal had the merit to be the first to propose tunability in a PFD. However, it is worth to say that there are also some experimental shortcomings, which reduces its usefulness in a real world application. Among them, we should mention first that, since two concatenated TFBGs are necessary (at least to reach a fractional differentiator order $n=1$ ), this requires continuously watching the spectral matching between both TFBGs. Second, the change of the polarization state usually involves mechanical movement, making the system cumbersome to operate. Third, it was not demonstrated that this proposal could reach low fractional differentiator orders (i.e., $0 \leq n \leq 0.81$ ). Finally, as it was discussed above, the amplitude behavior of the TFBG is far to resemble the required spectral filtering, which in turn necessarily adds processing errors. In order to take account of these items-especially the second one-the same group proposed 1 year later another alternative to tuned PFD (Shahoei, Albert, \& Yao, 2012), which we analyze below.

In their new proposal, Shahoei et al. (2012) demonstrated a continuously tunable PFD using a TFBG written in an $\mathrm{Er} / \mathrm{Yb}$ codoped fiber, where the fractional differentiator order $n$ is tuned by varying the pump power to the TFBG. The principle of operation can be succinctly explained as follows. As it was explained above, in a TFBG, the forward propagating core mode can couple either to a counter-propagating core mode or to several 
counter-propagating cladding modes; where each resonance wavelength is determined by (respectively):

$$
\begin{gathered}
\lambda_{\text {Bragg }}=2^{\text {core }} n_{\text {eff }} \Lambda_{g} / \cos \theta \\
\lambda_{\text {cladding }}=\left({ }^{\text {core }} n_{\text {eff }}+{ }^{\text {cladding }} n_{\text {eff }}\right) \Lambda_{g} / \cos \theta
\end{gathered}
$$

where $\theta$ is the tilt angle, $\Lambda_{g}$ is the nominal grating period, and ${ }^{\text {core }} n_{\text {eff }}$ and cladding $_{n_{\text {eff }}}$ are the effective refractive indices of the core mode $\left(\mathrm{LP}_{01}\right)$ and a particular cladding mode, respectively. Now, due to the high absorption of the $\mathrm{Er} / \mathrm{Yb}$ codoped fiber, the refractive index changes as a function of the pump power (Shahoei, Li, \& Yao, 2011). Therefore, according to Eqs. (21) and (22), the resonance wavelengths slightly change as a function of the pump power; which in turn also changes the phase variation around each resonance wavelength. Fig. 21 A and B shows both the response in amplitude and phase of an Er/Yb codoped TFBG, as a function of the pump power. Compared with their previous proposal (Li et al., 2011), the amplitude response has improved, resembling better the required characteristics given by Eq. (6). On the downside, the fractional order can be tuned in a slight margin, in one of the cladding resonances $1.25 \leq n \leq 1.72$, whereas in other cladding resonance $n=1$ and 0.95 . Apparently, it is difficult, as in their previous proposal, to reach low fractional orders with this approach. It is worth mentioning also, the necessity to shift in wavelength the signal to be
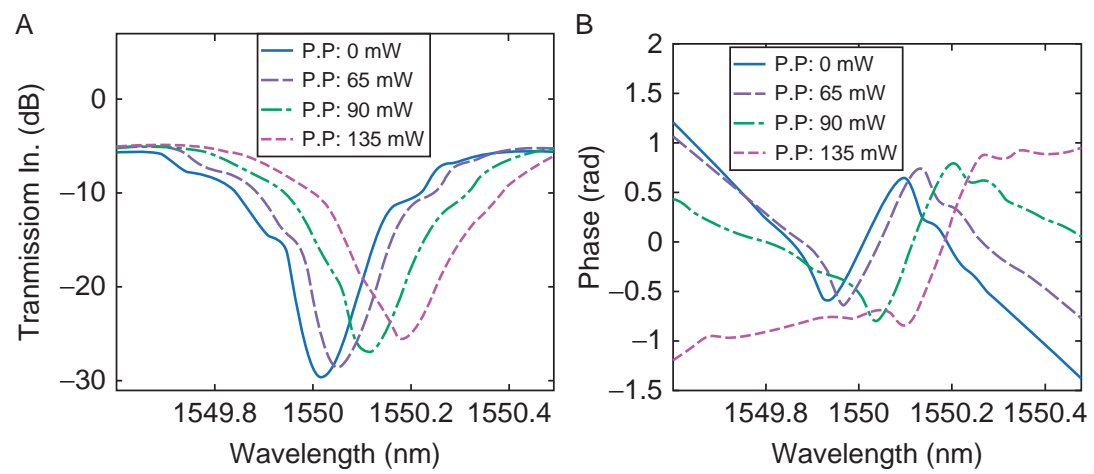

Fig. 21 (A) Magnitude and (B) phase responses of a cladding-mode resonance with a pumping power from 0 to $135-\mathrm{mW}$ (the reader should take into account that apparently there was a misprint in the original article; the units of the ordinate should be $\times \pi \mathrm{rad}$ ). PP: pumping power. From Shahoei, H., Albert, J., \& Yao, J. (2012). Tunable fractional order temporal differentiator by optically pumping a tilted fiber Bragg grating. IEEE Photonics Technology Letters, 24, 730-732. https://doi.org/10.1109/LPT.2012.2187331. 
processed as a function of the fractional differentiation order. This could represent a handicap for several applications. Finally, this proposal is able to process light pulses in the tens of gigahertz regime.

\subsubsection{The Long-Period Grating Approach}

The FBG approach to PFD is generally well suited for lower operation bandwidths (in the tens of gigahertz regime). The PFD of shorter light pulses (well in the THz regime) is best processed with LPGs (Cuadrado-Laborde \& Andrés, 2011). An in-fiber LPG induces gradual coupling between the fundamental mode and copropagating cladding modes as light propagates along the fiber length $z$; unlike in FBGs, where there is a counter-propagating coupling between modes. The amplitude evolution of a pulse propagating in an LPG for both core and cladding modes can be described through the following matrix (Erdogan, 1997):

$$
\left[\begin{array}{l}
A_{1}(z, \omega) \\
A_{2}(z, \omega)
\end{array}\right]=\left[\begin{array}{ll}
F_{11}(z, \omega) & F_{12}(z, \omega) \\
F_{21}(z, \omega) & F_{22}(z, \omega)
\end{array}\right]\left[\begin{array}{l}
A_{1}(0, \omega) \\
A_{2}(0, \omega)
\end{array}\right],
$$

where $A_{i}(0, \omega)=\mathfrak{J}\left[a_{i}(0, t)\right], a_{i}(0, t)$ is the temporal amplitude of the pulse at the beginning of the LPG $(z=0)$ and the subindex $i=1,2$ denotes the core and cladding modes, respectively. If the index modulation depth and the periodicity are constants over the whole grating, each matrix element in Eq. (23) has the following expressions:

$$
\begin{aligned}
& F_{11}(z, \omega)=\left[\cos (\gamma z)+j \sigma \gamma^{-1} \sin (\gamma z)\right] \exp \left[j\left(\beta_{1}-\sigma\right) z\right], \\
& F_{12}(z, \omega)=j \kappa \gamma^{-1} \sin (\gamma z) \exp \left[j\left(\beta_{1}-\sigma\right) z\right], \\
& F_{21}(z, \omega)=j \kappa \gamma^{-1} \sin (\gamma z) \exp \left[j\left(\beta_{2}+\sigma\right) z\right], \\
& F_{22}(z, \omega)=\left[\cos (\gamma z)-j \sigma \gamma^{-1} \sin (\gamma z)\right] \exp \left[j\left(\beta_{2}+\sigma\right) z\right],
\end{aligned}
$$

where $\kappa$ is the coupling coefficient, $2 \sigma(\omega)=\beta_{1}(\omega)-\beta_{2}(\omega)-2 \pi / \Lambda, \beta_{1}(\omega)$ and $\beta_{2}(\omega)$ are the propagation constants for the core and the cladding modes, respectively; $\Lambda$ is the grating period and $\gamma^{2}=\kappa^{2}+\sigma^{2}$ (Erdogan, 1997). If only the fundamental mode is initially excited by the pulse launched at the input of the LPG, i.e., $A_{2}(0, \omega)=0$ (see Eq. 23). Then the temporal pulse envelope propagating through the core at a distance $z$ along the LPG can be obtained by $a_{1}(z, t)=\mathfrak{I}^{-1}\left[A_{1}(z, \omega)\right]=\mathfrak{J}^{-1}\left[F_{11}(z, \omega) A_{1}(0, \omega)\right]$ (see Eq. 23), where $\mathfrak{I}^{-1}$ stands for the inverse Fourier transform. In this sense, $F_{11}(z, \omega)$ can be considered the transfer function $H(\omega)$ of our device; whose phase is given by (Poveda-Wong et al., 2016b):

$$
\theta(\gamma)=\frac{\pi}{2} \operatorname{sgn}\left[\frac{\sigma}{\gamma} \sin (\gamma z)\right]-\arctan \left(\frac{\gamma}{\sigma} \cot (\gamma z)\right)+\left(\beta_{1}-\sigma\right) z,
$$


where sgn[.] is the signum function. The maximum phase change $\Delta \theta$, can be obtained by finding the roots of the first derivative of Eq. (25), which are given by $\gamma z=\pi / 2$. By substituting this value in Eq. (25) and taking into account that $\Delta \theta=n \times \pi$, an analytical expression for the fractional order $n$ as a function of the LPG parameters is obtained, i.e.,

$$
n=1-\sqrt{1-(z / L)^{2}}
$$

where $L$ is the LPG length providing full energy coupling into the cladding mode, i.e., $\kappa L=\pi / 2$. (Additionally, in obtaining Eq. (26), the term $\beta_{1} z$ was omitted from Eq. (25), since it is only responsible for a time delay in the output signal.) The simplicity of this expression is remarkable, especially when taking into account that no approximation was used. It is worth to mention also, the generality of Eq. (26), i.e., every LPG designed to perform a full-energy coupling into the cladding mode, behaves as a fractional-order differentiator at fractions of its characteristic grating length $L$. Being the fractional order $n$ only related with this fraction of the grating length. When $z=L \rightarrow n=1$ (see Eq. 26); and in this situation the phase shift $\Delta \theta=\pi$, and the transmission varies linearly with the baseband frequency around $\omega=0$, as it is required for a first-order differentiator (Slavík, Park, Kulishov, Morandotti, \& Azaña, 2006). On the other hand, at fractions of $L$, the spectral behavior of the LPG approaches that of a fractional-order differentiator filter (see Eq. 6). In this way, an optical pulse propagating in the core mode of a LPG designed for full energy coupling $(\kappa L=\pi / 2)$ experiences all the fractional derivatives between $n=0$ (for $z=0$ ) and $n=1$ (for $z=L$ ) while propagating through the LPG. It is worth to remember here the work of Mendlovic and Ozaktas (1993a, 1993b) on FFTs, which will be described in Section 4. They demonstrated that if a graded index media (GRIN) is constructed to perform a Fourier transform at a length $L$, then at fractions of $L$, the obtained field is indeed the FFT. In a similar fashion to the result obtained later by Cuadrado-Laborde and Andrés (2011) for fractional differentiation in LPGs, except that here the relationship is not linear (see Eq. 26).

Fig. 22A shows graphically the relationship given in Eq. (26). The amplitude and phase responses of the LPG are shown in Fig. 22B and C, at different fractions of the LPG length given by $z / L=1,0.954$, and 0.866. By using Eq. (26), these fraction lengths correspond to the following fractional differentiator orders $n=1,0.7$, and 0.5 , respectively. (Further details of this simulation can be obtained from the original work of Poveda-Wong et al., 2016b.) For example, when $z / L=0.866(n=0.5)$, 

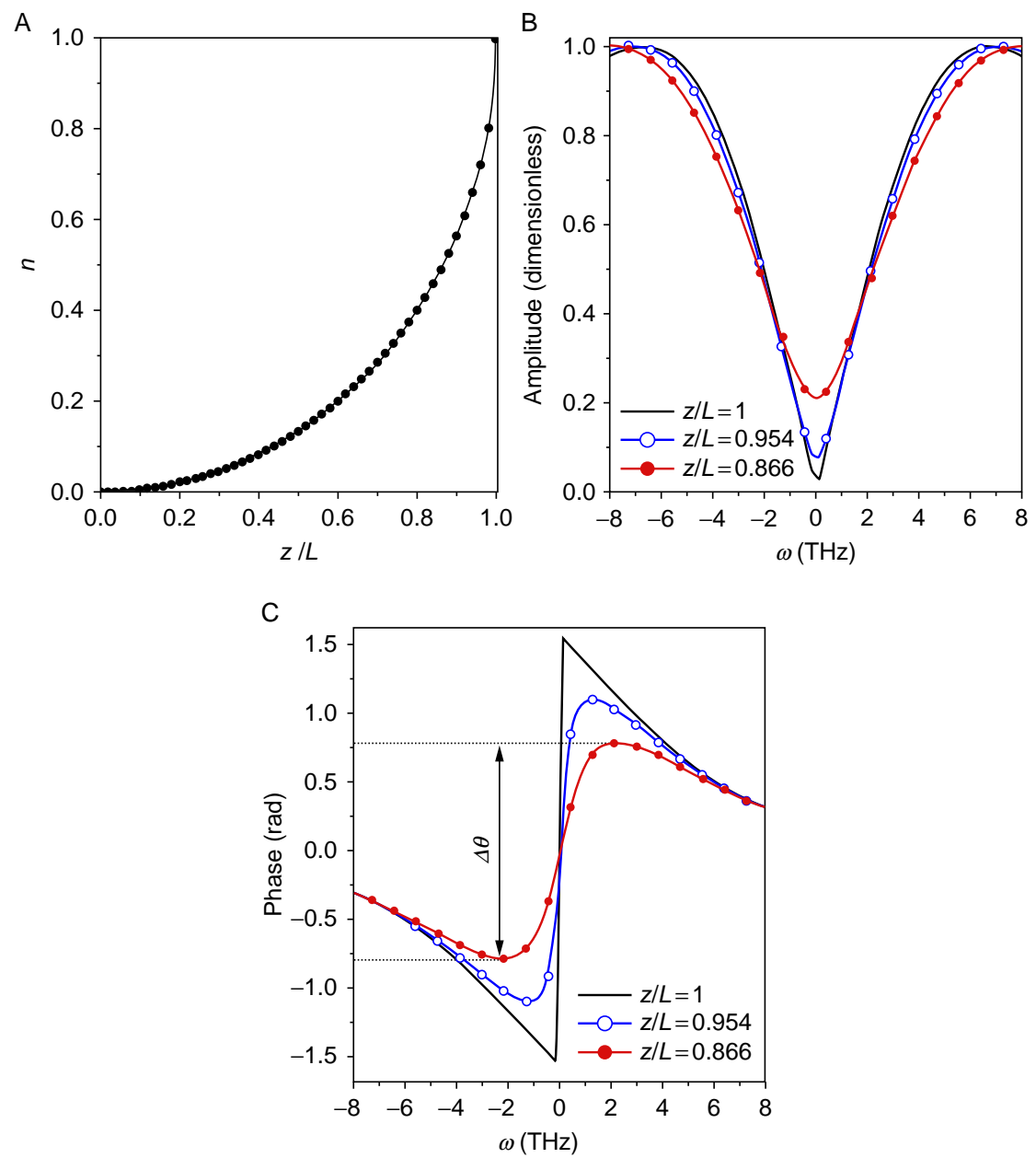

Fig. 22 (A) Fractional order of differentiation $n$ as a function of the fraction of grating length $z / L$. (B) Amplitude and (C) phase response of the LPG at different fractions of the grating length L. From Poveda-Wong, L., Carrascosa, A., Cuadrado-Laborde, C., Cruz, J. L., Díez, A., \& Andrés, M. V. (2016b). Long-period grating assisted fractional differentiation of highly chirped light pulses, Optics Communications, 363, 37-41, https://doi.org/10.1016/j. optcom.2015.10.063.

the maximum phase change is $\Delta \theta=1.57 \mathrm{rad}$, see the dotted lines in Fig. 22C, which match exactly with the expected phase change of a 0.5 th-order fractional differentiator.

This LPG proposal was corroborated experimentally by Poveda-Wong et al. (2016b) by recording an LPG in a boron-doped photosensitive fiber 
(PS980 by Fibercore, numerical aperture of 0.13 and a cut-off wavelength of $980 \mathrm{~nm}$ ) by using the point-by-point technique. The selected periodicity was $187.6 \mu \mathrm{m}$, with a final LPG length of $146.5 \mathrm{~mm}$. The transmission response of the LPG was followed during the fabrication process by registering the transmitted light provided by an led source in an optical spectrum analyzer. The LPG fabrication was interrupted when the transmission reached a resonance depth of $14 \mathrm{~dB}$, with a $3 \mathrm{~dB}$ bandwidth of $1.14 \mathrm{~nm}$ (see Fig. 23A). This resonance depth was below the full coupling condition, being equivalent to a working condition of $z / L \cong 0.86$, which in turn corresponds to a 0.5 thorder fractional differentiator. In the same figure, the theoretical intensity response of an ideal 0.5 th-order fractional differentiator filter was superimposed (see Eq. 6). The degree of similarity between both traces is reasonably good within the whole operational optical bandwidth, except at the
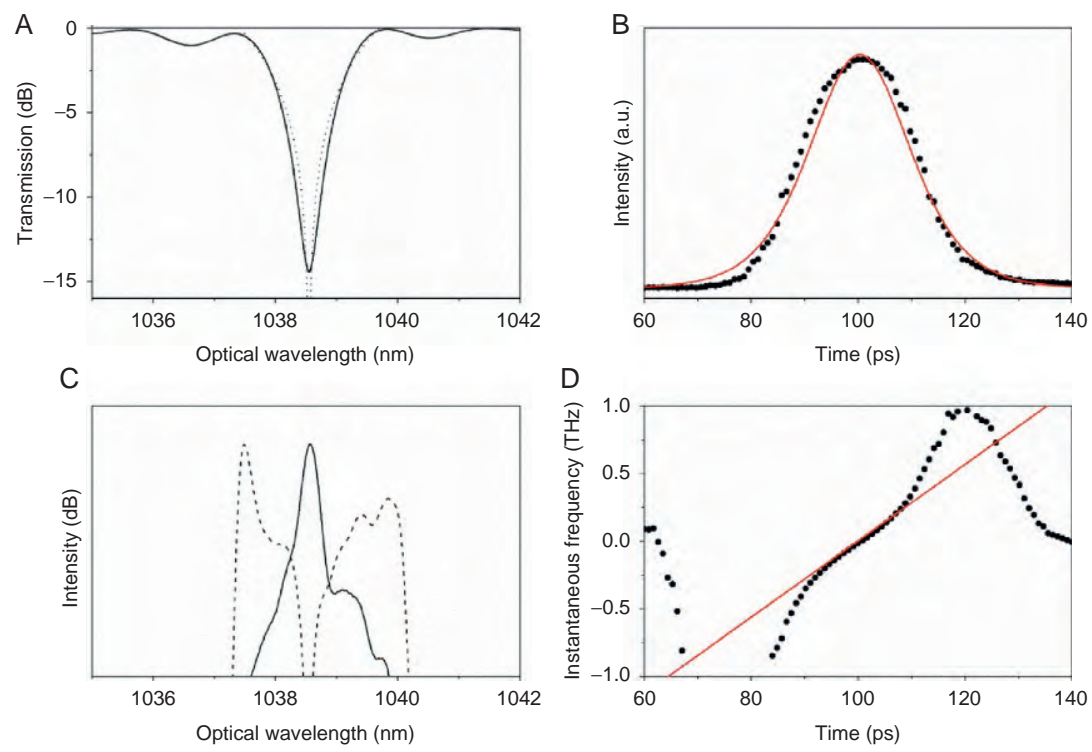

Fig. 23 (A) Measured optical spectrum of the LPG and theoretical intensity response of a 0.5 th-order fractional differentiator, solid and dashed lines, respectively. (B) Measured output light pulse of the mode-locked laser with its corresponding fitting, scatter points and solid curve, respectively. (C) Measured spectra of the light pulses at the input and output of the LPG, solid and dotted lines, respectively. (D) Measured instantaneous frequency of the output light pulse of the mode-locked laser, with its corresponding linear fitting with chirp $C=-30$, scatter points and solid curve, respectively. From Poveda-Wong, $L$., Carrascosa, A., Cuadrado-Laborde, C., Cruz, J. L., Díez, A., \& Andrés, M. V. (2016b). Long-period grating assisted fractional differentiation of highly chirped light pulses, Optics Communications, 363, 37-41, https://doi.org/10.1016/j.optcom.2015.10.063. 
resonance frequency, where the theoretical transmission decays to $-\infty$ (as opposed to the LPG transmission which decays to $-14 \mathrm{~dB}$ ). These slight deviations in the magnitude response of the proposed filter to perform the fractional differentiation (as compared with the ideal filter) are typical for these setups. And they have minor consequences for the same deviation in the phase response, something which has been discussed with great detail in the work of Oppenheim and Lim (1981). On the other hand, the light pulses to be optically differentiated were provided by a passively mode-locked ytterbium fiber laser, which can be approximately fitted with an hyperbolic secant profile $f(t)=\operatorname{sech}\left(t / T_{0}\right)$, with $T_{0}=13$ ps, i.e., a FWHM of 23 ps (see Fig. 23B). Fig. 23C shows the spectra of the signals at the input and output of the LPG. One can see that the input signal carrier is well aligned with the resonance optical wavelength of the LPG. As a result, the optical carrier is deeply suppressed at the output spectrum, as expected for a photonic differentiator, regardless being fractional or not, since a PFD is a high pass filter. The pulses provided by the all-fiber mode-locked laser are far from being transform limited. The authors have measured its phase profile by a previously developed technique, also developed by the same group (Cuadrado-Laborde et al., 2014), resulting in a measurement of a linear chirp of -30 (see Fig. 23D). This fact is very important, since the output temporal waveform is deeply influenced by the spectral content. The influence of chirp on the temporal waveform after a PFD was further studied with more detail in CuadradoLaborde, Lancis, and Andrés (2010).

Fig. 24A shows the measured temporal intensity profiles of the light pulse at the output of the LPG. In the same figure, the simulated response of an ideal 0.5 th-order fractional differentiator is also shown by composing an input pulse with the square root of the measured temporal intensity profile as the modulus, and the reconstructed phase obtained above for $f(t)$. The instantaneous frequency of the light pulses at the output of the LPG, i.e., $\mathrm{d}^{n} f(t) /\left.\mathrm{d} t^{n}\right|_{n=0.5}$, was measured by using the temporal intensity profiles of both, the originally fractional differentiated and further propagated pulses registered by the oscilloscope (see Fig. 24B). In the same figure, we also have shown a calculated instantaneous frequency for the simulated response and propagation of the ideal 0.5 th-order fractional differentiator, shown in Fig. 24A. The peak in the instantaneous frequency profile corresponding to the intensity minima between the peaks of intensity maxima (localized at $\sim 100 \mathrm{ps}$ ), is a consequence of the phase difference $\Delta \theta=0.5 \times \pi$ between the right and left peaks of the differentiated pulses, which in turn is a 

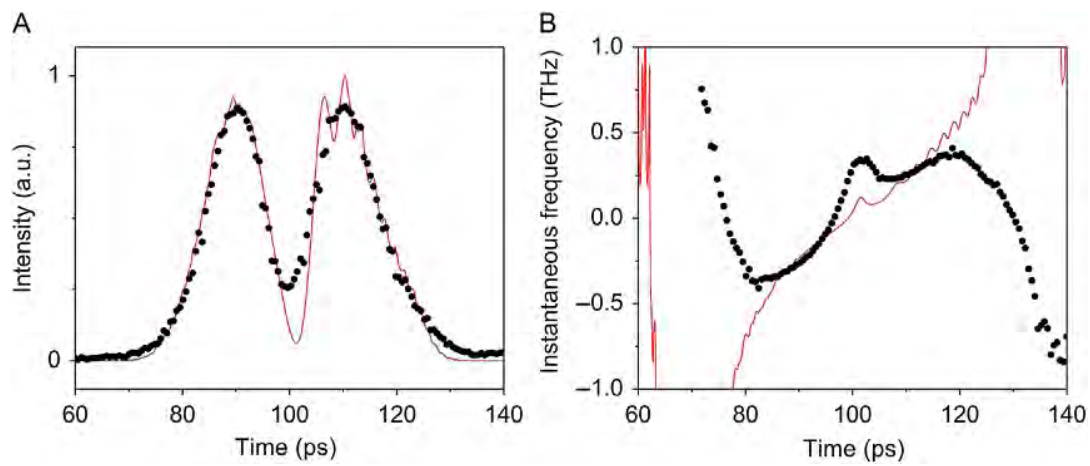

Fig. 24 (A) Measured temporal intensity profile of the light pulse at the output of the LPG and simulated response of an ideal 0.5 th-order fractional differentiator, scatter points and solid curve, respectively. (B) Measured instantaneous frequency of the light pulse at the output of the LPG and calculated instantaneous frequency for the simulated response of an ideal 0.5 th-order fractional differentiator, scatter points and solid curve, respectively. From Poveda-Wong, L., Carrascosa, A., Cuadrado-Laborde, C., Cruz, J. L., Díez, A., \& Andrés, M. V. (2016b). Long-period grating assisted fractional differentiation of highly chirped light pulses, Optics Communications, 363, 37-41, https://doi.org/10. 1016/j.optcom.2015.10.063.

consequence of the fractional differentiation (see Fig. 24B). It is interesting to point out that if the LPG would have a frequency response of the type $H(\omega)=\left|(j \omega)^{n}\right|$-i.e., similar only in intensity to a fractional-order differentiator filter response, but without a phase discontinuity at the resonance frequency - the measured instantaneous frequency profile would be linear, i.e., without an instantaneous frequency peak in the intensity minima between the peaks of intensity maxima.

\subsubsection{The Microring Resonator Approach}

Silicon microring resonators (MRR) have been also proposed for PFD. The first to propose a MRR-based device for this task were Shahoei, Xu, Schmid, and Yao (2013). They implemented a tunable fractional-order differentiator with a SOI MRR combined with a MMI coupler. Through changing the input polarization state, the self-coupling coefficient $t$, and the loss factor $\alpha$ of the MRR with the MMI coupler are changed. Correspondingly, the coupling regime is changed. Through changing the coupling regime from overcoupled to undercoupled, the phase shift in the resonance wavelength is changed. The device is schematically shown in Fig. 25A; whereas in Fig. 25B a detail of the MMI coupler is shown, where 


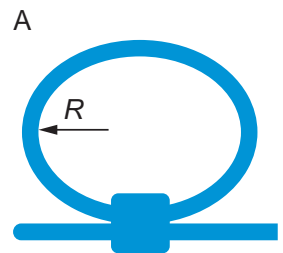

B

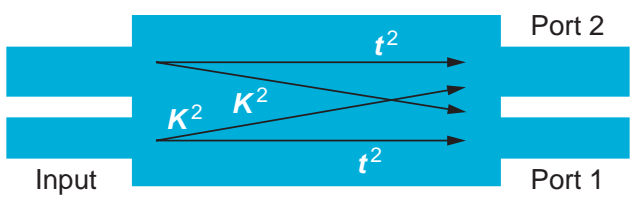

Fig. 25 (A) Microring resonator with an MMI coupler and (B) a close-up view of the MMI coupler. From Shahoei, H., Xu, D. X., Schmid, J. H., \& Yao, J. (2013). Photonic fractional-order differentiator using an SOI microring resonator with an MMI coupler. IEEE Photonics Technology Letters, 25, 1408-1411. https://doi.org/10.1109/LPT.2013.2266252.

the power-splitting ratios of the MMI fulfill $t^{2}+K^{2}=1$. This phase shift variation $\Delta \theta_{n}$ corresponds to different fractional orders, since $\Delta \theta_{n}=n \times \pi$. The transmissivity of a MRR can be expressed as (Yariv, 2000):

$$
T=\alpha_{\mathrm{MMI}} \frac{t-\alpha \exp (j \theta)}{1-\alpha t \exp (j \theta)},
$$

where $\theta=2 \pi n_{\text {eff }} L / \lambda$ is the phase accumulated during one complete round trip of length $L, \alpha=\alpha_{\mathrm{MMI}} \alpha_{\text {ring }}$ is the combined field amplitude loss factor including both ring propagation loss $\alpha_{\text {ring }}$ and the MMI coupler loss $\alpha_{\mathrm{MMI}}$, $n_{\text {eff }}$ is the waveguide effective index, and $\lambda$ is the optical wavelength. This transmissivity has a twofold dependence on $\alpha_{\mathrm{MMI}}$, in amplitude and phase. Although it can be obtained from Eq. (27), the authors did not provide an analytical expression for the fractional order as a function of the combined loss; i.e., $n=f(\alpha)$. Instead, they provide a complete simulation study by keeping $\alpha=0.77$ fixed and varying $t$ (see Fig. 26A and B). The opposite was done in Fig. 26C and D, where $t=0.77$ remained fixed and $\alpha$ was varied; whereas in Fig. 26E and $\mathrm{F}$ both $t$ and $\alpha$ were varied.

From the simulations shown in Fig. 26, it is clear that different fractional orders below and above the unity can be obtained, by changing the input polarization state of the analyzed light pulse. The authors fabricated this MMI-coupled MRR on an SOI wafer; further details can be found in the original publication by Shahoei et al. (2013). Fig. 27A shows the measured transmission spectrum of the ring (perimeter of $300 \mu \mathrm{m}$ ) at the resonance wavelength of $1558 \mathrm{~nm}$. The measured phase response of the MRR around the resonance is shown in Fig. 27B. The simulated magnitude and phase responses were also shown for comparison, by using $t=0.804$ and $\alpha=0.75$ (information about how these two parameters were measured was not provided by the authors). When the input polarization state is changed, 

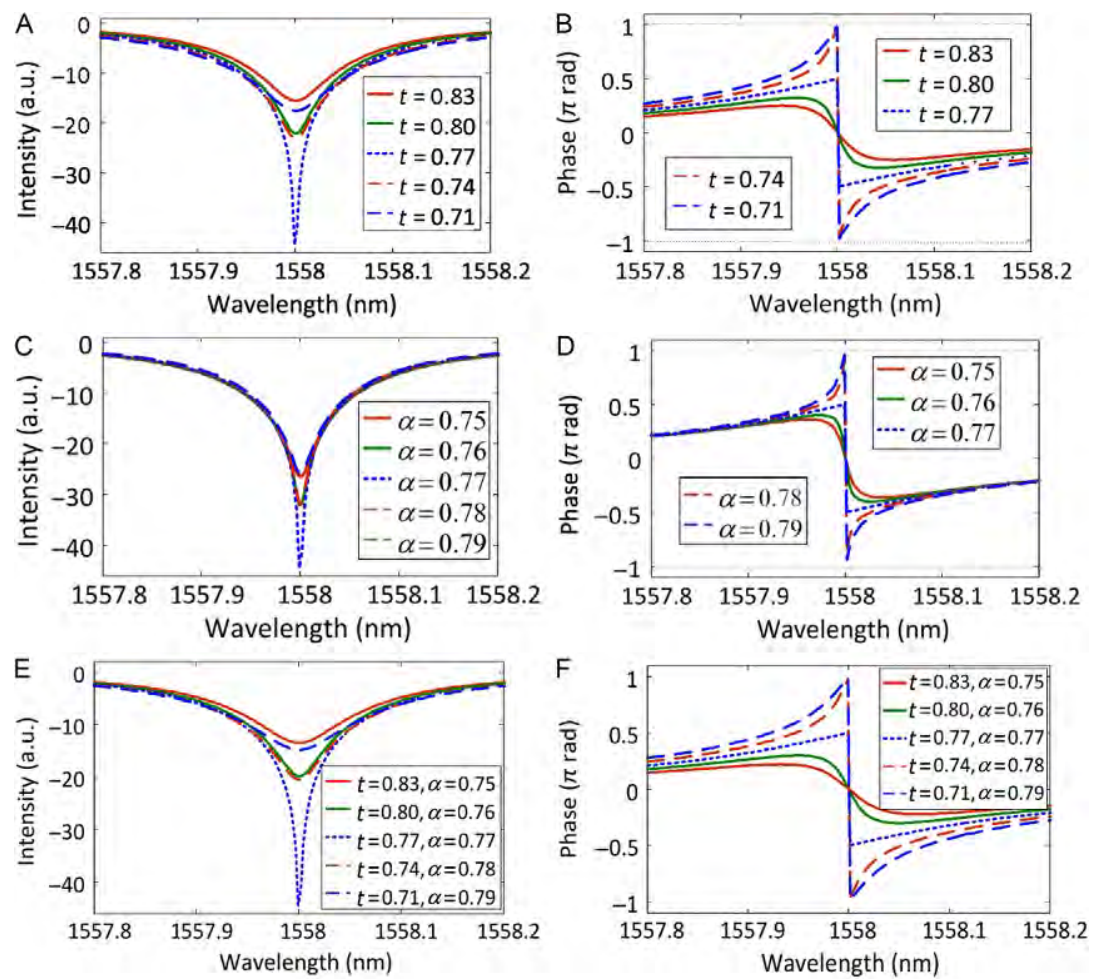

Fig. 26 Simulated transmission magnitude and the corresponding phase for (A and $B$ ) $\alpha=0.77$ and $t=0.83,0.8,0.77,0.74,0.71$, (C and $\mathrm{D}) t=0.77, \alpha=0.75,0.76,0.77,0.78,0.79$, and (E and F) different $t$ and $\alpha$. From Shahoei, H., Xu, D. X., Schmid, J. H., \& Yao, J. (2013). Photonic fractional-order differentiator using an SOI microring resonator with an $\mathrm{MMI}$ coupler. IEEE Photonics Technology Letters, 25, 1408-1411. https://doi.org/10.1109/LPT.2013. 2266252.

both the transmission intensity and the phase response changes; this was shown in Fig. 27C and D. It is clear from this experiment that the fractional order can be tuned around unity, by changing the input polarization state of the incident light pulse.

Finally, Shahoei et al. (2013) dynamically tested their proposal with the setup shown in Fig. 28. The tandem mode-locked laser plus wave shaper, produces Gaussian light pulses of $22 \mathrm{ps}$ time width at $1558 \mathrm{~nm}$, which could be moderately chirped (the reported spectral width- $45 \mathrm{GHz}$ - exceeds the time-bandwidth product for Gaussian pulses). An EDFA was also used to increase the signal-to-noise ratio-this is especially relevant for the output light pulses, due to the typically low energetic efficiency of PFDs. At the output of the EDFA, and before the MMI-coupled MRR, a polarizer and a PC 
A

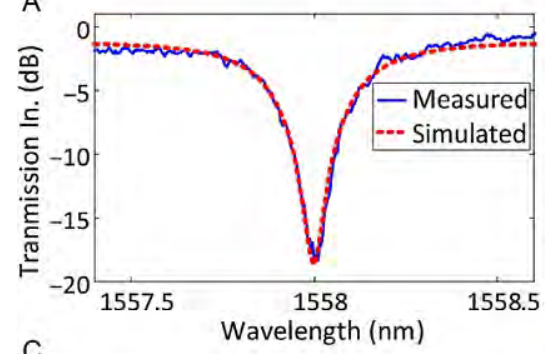

C

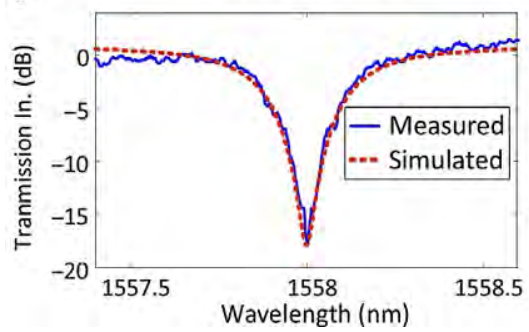

B
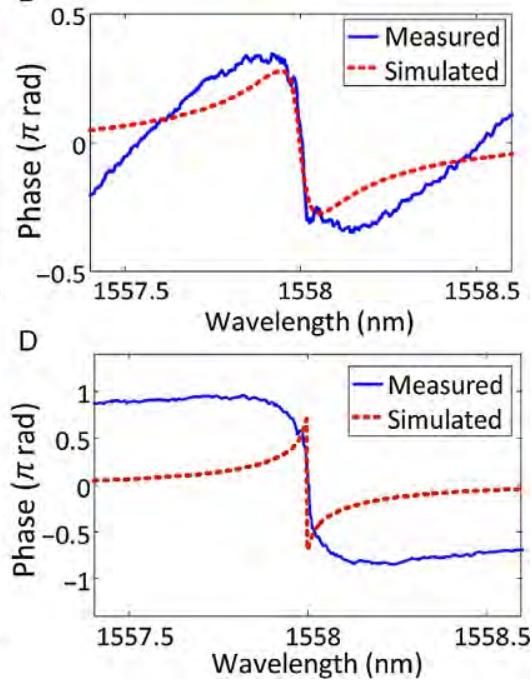

Fig. 27 The magnitude ( $A$ and $C$ ) and phase response ( $B$ and $D$ ) of the ring operating in transmission at $1558 \mathrm{~nm}$ for two different input polarization states. The coupling regime for the first polarization state is the overcoupled regime and the second polarization state is changed to the undercoupled regime. The solid lines show the measured spectrums and the dashed lines show the simulated spectrums. From Shahoei, $H_{\text {., }} X u, D$. X., Schmid, J. H., \& Yao, J. (2013). Photonic fractional-order differentiator using an SOI microring resonator with an MMI coupler. IEEE Photonics Technology Letters, 25, 1408-1411. https://doi.org/10.1109/LPT.2013.2266252.

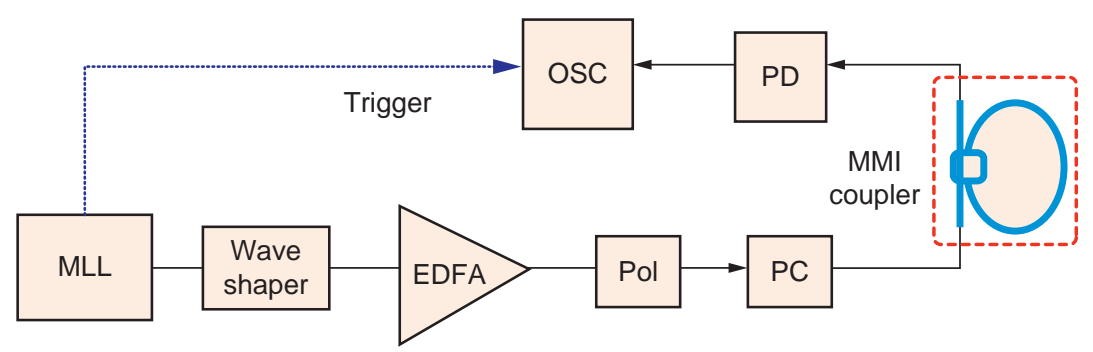

Fig. 28 Experimental setup. $M M L$ : mode-locked laser; Pol: polarizer; $P C$ : polarization controller; PD: photodetector; and OSC: oscilloscope. From Shahoei, H., Xu, D. X., Schmid, J. H., \& Yao, J. (2013). Photonic fractional-order differentiator using an SOI microring resonator with an MMI coupler. IEEE Photonics Technology Letters, 25, 1408-1411. https://doi.org/10.1109/LPT.2013.2266252. 
were placed, in order to change the fractional order of differentiation. The shaped pulse at the output of the wave shaper is shown in Fig. 29A. An ideal Gaussian pulse with the same temporal width (22 ps) is also shown in Fig. 29A for comparison. As can be seen, the experimentally generated input pulse is close to a Gaussian except a slight deviation in the front tail. The polarization state of the input pulse is controlled by tuning the PC. The differentiated pulse is detected by the photodetector, and the waveform is observed by an oscilloscope. A differentiated pulse with an order of $n=1,1.2,1.5,0.67$, and 0.37 is generated and shown in Fig. 29B-F, respectively. Simulated pulses with an ideal input Gaussian pulse and an ideal differentiator with the same orders

A

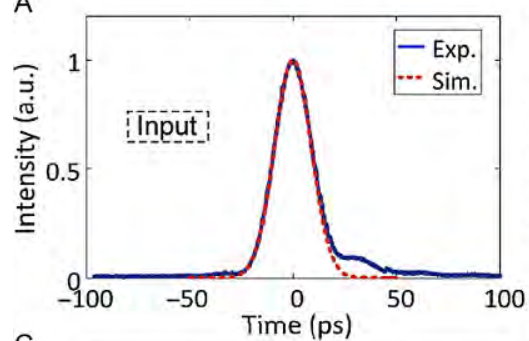

C
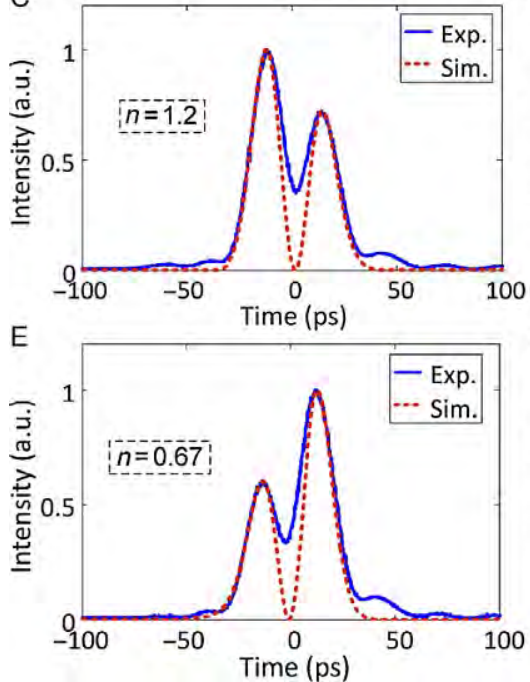

B
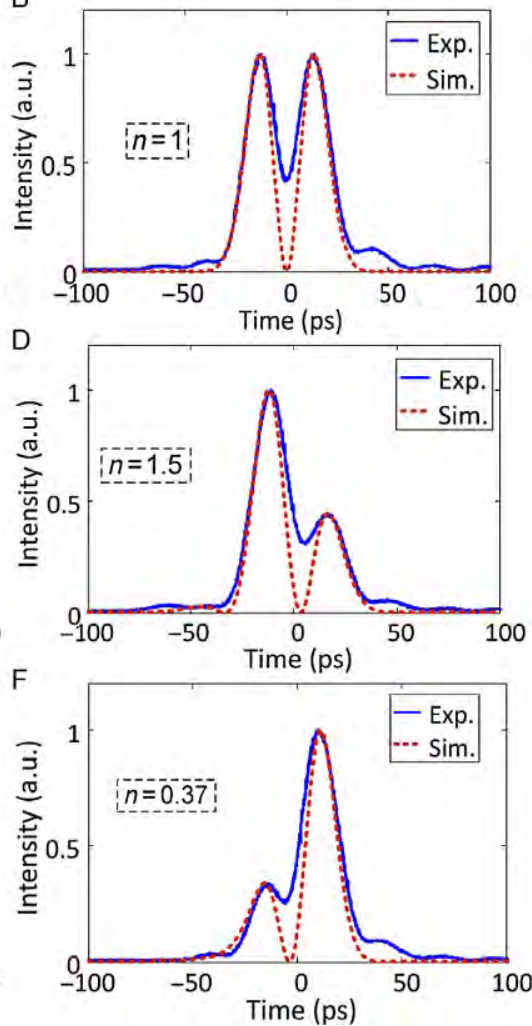

Fig. 29 (A) An input Gaussian pulse with an FWHM of $22 \mathrm{ps,} \mathrm{and} \mathrm{the} \mathrm{differentiated}$ pulses at the different input polarization states corresponding to differentiation orders of (B) $n=1$, (C) $n=1.2$, (D) $n=1.5$, (E) $n=0.67$, and (F) $n=0.37$. From Shahoei, H., Xu, D. X., Schmid, J. H., \& Yao, J. (2013). Photonic fractional-order differentiator using an SOI microring resonator with an MMI coupler. IEEE Photonics Technology Letters, 25, 1408-1411. https://doi.org/10.1109/LPT.2013.2266252. 
are also shown in Fig. 29B-F, as dashed lines. As can be seen a good agreement is achieved between the experimentally generated pulses and the pulses with an ideal PFD. Unfortunately, this tuning in the fractional order cannot be controlled a priori, being ultimately determined by comparison between the experimentally obtained and the theoretical waveforms. In contrast to this behavior, in the LPG approach previously explained in Section 2.2.1 (Poveda-Wong et al., 2016b), the fractional order is precisely determined during the fabrication process by monitoring the optical transmittance.

The second SOI MRR proposal for fractional differentiation came from Zheng, Dong, et al. (2014). As an improvement, in their approach, it is not necessary to use a polarizer plus a polarization control. Because in this case, the tuning in fractional order is not reached by changing the polarization state of the incident light, but by modifying the applied voltage on the MRR. Again, the transmissivity of a MRR can be expressed in Eq. (27); except these authors used a different nomenclature for the transmission coefficient $(r$, instead of $t)$ and there is not a MMI coupler. Thus, the transmittance can be expressed as (Yariv, 2000):

$$
T=\frac{r-\alpha \exp (j \theta)}{1-\alpha \exp (j \theta)}
$$

where $\theta=2 \pi n_{\text {eff }} L / \lambda$ is the phase accumulated during one complete round trip of length $L, \alpha$ is the ring propagation loss factor, $n_{\mathrm{eff}}$ is the waveguide effective index, $\lambda$ is the optical wavelength, and $r$ is the transmission coefficient. There exist an inverse dependence between the applied voltage on the doped silicon MRR and the ring propagation loss factor $\alpha$. Therefore, the phase discontinuity at the resonance wavelength can be tuned by varying the applied voltage. A relationship between $n$ and $\alpha$ can be obtained by using Eq. (28). However that information was not provided by the authors. Instead, these authors simulated the response in both amplitude and phase of the transmissivity, given in Eq. (28). In these simulations, the transmission coefficient remained fixed, since it did not change as the applied voltage varies. This information was essentially very similar to the simulations shown above for the work of Shahoei et al. (2013) (see Fig. 26); for this reason, it will not be repeated here. These authors designed and fabricated an on-chip MRR structure to demonstrate the viability of their proposal (further details can be found in their original work). Fig. 30A shows the micrographs of the fabricated MRR and Fig. 30B shows the zoom-in ring region. The bottom of Fig. 30 shows the measured magnitude response of the MRR for applied 

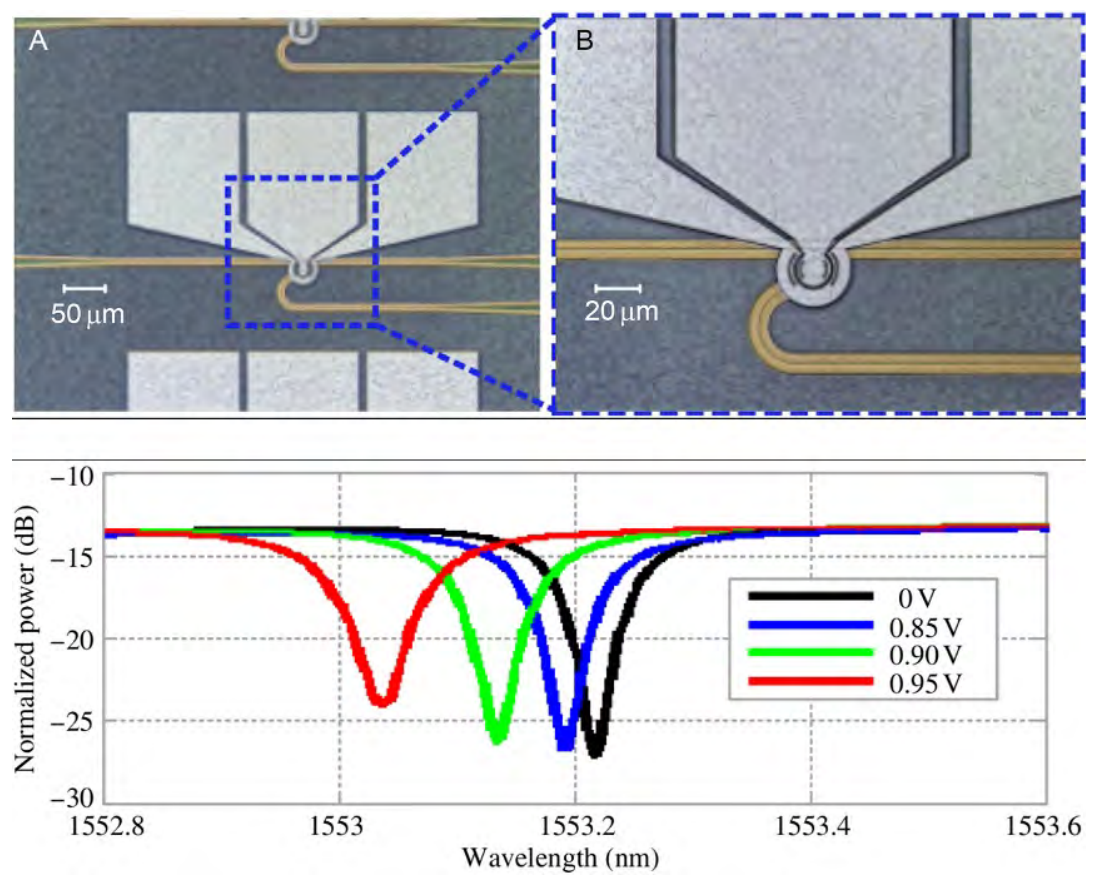

Fig. 30 Top (A) Microscope image of the fabricated MRI and (B) microscope image of the zoom-in ring region. Bottom: Measured magnitude response of the MRR in transmission at different voltages. From Zheng, A., Dong, J., Zhou, L., Xiao, X., Yang, Q., Zhang, X., \& Chen, J. (2014b). Fractional-order photonic differentiator using an on-chip microring resonator. Optics Letters, 39, 6355-6358. https://doi.org/10.1364/OL.39.006355.

voltages on the MRR in the range $0-0.95 \mathrm{~V}$. The phase response was not measured; however, the authors associated the deepest notch in amplitude (at $0 \mathrm{~V}$ ) with the $\pi$ phase shift by comparison with their simulations (corresponding to fractional differentiation order $n=1$ ). It should be pointed out, as a drawback, that the operation wavelength does not remain fixed as the differentiation order is changed. These authors dynamically tested their device, by using as input light signal a Gaussian pulse train (we believe transform limited, although this information was not provided) with a FWHM of 30 ps and a repetition frequency of $10 \mathrm{GHz}$, as shown in Fig. 31A. Next, as the applied voltage on the MRR increased from 0 to $0.95 \mathrm{~V}$, the intensity temporal waveforms were measured; this information is shown in Fig. 31B-E. They found, that these measured waveforms correspond to the fractional differentiations orders $n=0.97,0.80,0.63$, and 0.58 , respectively. 


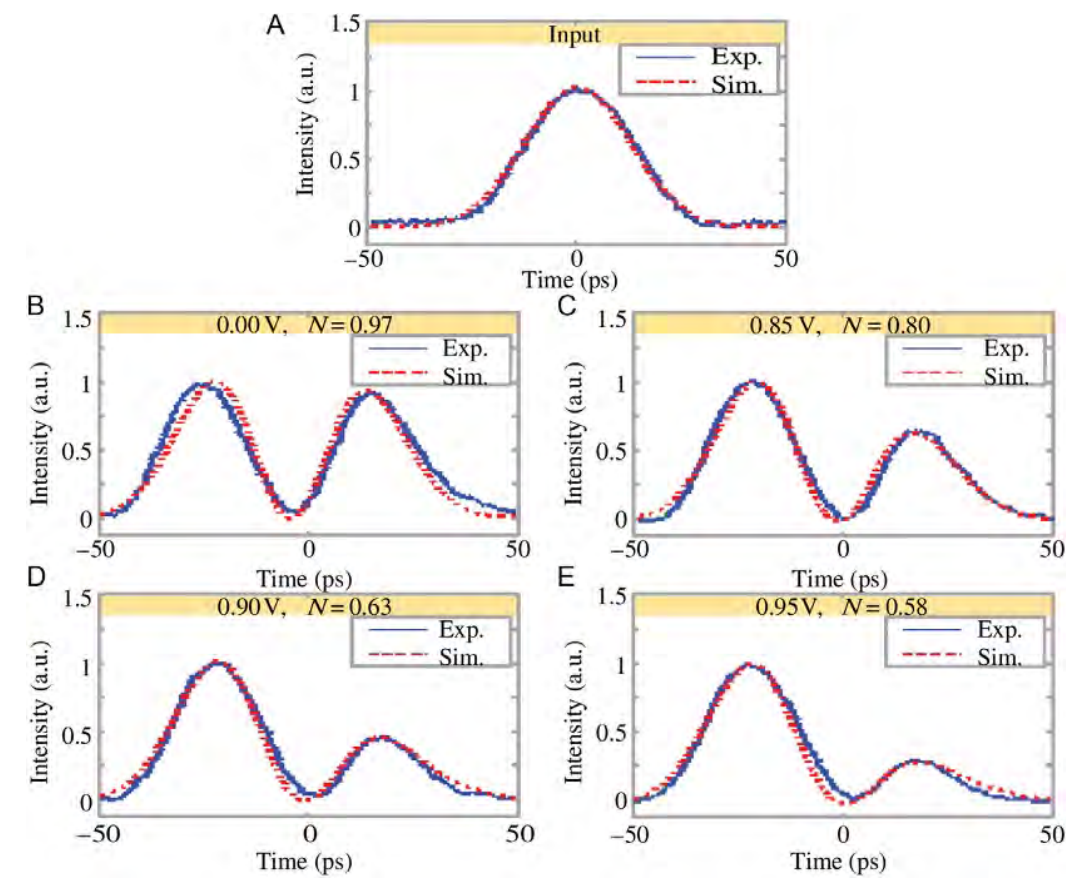

Fig. 31 (A) Input Gaussian pulse with an FWHM of $30 \mathrm{ps,} \mathrm{and} \mathrm{the} \mathrm{differentiated} \mathrm{pulses} \mathrm{at}$ the different voltages corresponding to differentiation orders of (B) $n=0.97,(C) n=0.80$, (D) $n=0.63$, and (E) $n=0.58$. From Zheng, A., Dong, J., Zhou, L., Xiao, X., Yang, Q., Zhang, X., \& Chen, J. (2014b). Fractional-order photonic differentiator using an on-chip microring resonator. Optics Letters, 39, 6355-6358. https://doi.org/10.1364/OL.39.006355.

\subsection{Photonic Fractional Integration}

To the best of our knowledge, Cuadrado-Laborde and Andrés (2010) were the first to discuss the possibility to perform a PFI. In the following, we outline their principal conclusions.

A real system cannot perform the ideal impulse response required for PFI; i.e., $h(t) \propto(t)^{n-1}$ (see Eq. 4), because of the divergence at $t=0$, when $0<n<1$. However, we can approach accurately this ideal impulse response with a filter with the following approximate impulse response:

$$
\widetilde{h}(t)=A \tau^{1-n}(t+\tau)^{n-1}
$$

where $0<t<T, A$ is an amplitude constant, $T$ is the operational time window, and $\tau$ is a positive time constant provided to avoid the divergence at $t=0$ (of course, if $\Delta t$ is the input signal time width; then $\tau \ll \Delta t$ for an 
accurate operation). Within the limit of the well-known first-order Born approximation, this approximate impulse response can be reached by a conveniently apodized FBG operated in reflection (Azaña \& Chen, 2002). Therefore, for a sufficiently weak grating, the corresponding reflection impulse response is proportional to the apodization profile of the index modulation depth. Thus, according to Eq. (29) and by the space-to-time mapping $t \rightarrow 2 z n_{a v} /-$ where $c$ is the speed of light in vacuum, $z$ is the distance along the fiber, and $n_{a v}$ is the average refractive index of the grating, the apodization profile should vary as:

$$
\Delta n(z)=\Delta n_{\max } z_{s}^{1-n}\left(z+z_{s}\right)^{n-1}
$$

with $0<z<L, L=c T /\left(2 n_{a v}\right)$ is the total grating length, $z_{s}=c \tau /\left(2 n_{a v}\right)$ is the spatial shift provided to avoid the divergence at $z=0$, and $\Delta n_{\max }$ is the maximum index modulation depth. Additionally, the spatial shift $z_{\mathrm{s}}$ was set to be an integer number of the grating period $\Lambda=\lambda_{\mathrm{B}}\left(2 n_{\mathrm{eff}}\right)^{-1}$, with $\lambda_{\mathrm{B}}$ the Bragg wavelength $(1550 \mathrm{~nm})$, and $n_{\mathrm{eff}}$ is the modal effective index (1.452). Fig. 32A exemplifies the functional relationship between the fractional order of integration and the apodization profile described by Eq. (30), for several $n$ values between 0 and $1, L=21 \mathrm{~mm}, z_{s}=60 \times \Lambda$, and $\Delta n_{\max }=1.5 \times 10^{-4}$. As can be observed, an $n$ th-order fractional integral operation implies a $\sim z^{n-1}$ dependence for the index modulation depth. Furthermore, when $n=1-$ i.e., a first-order integrator-according to Eq. (30) $\Delta n(z)=\Delta n_{\max }$-i.e., a constant
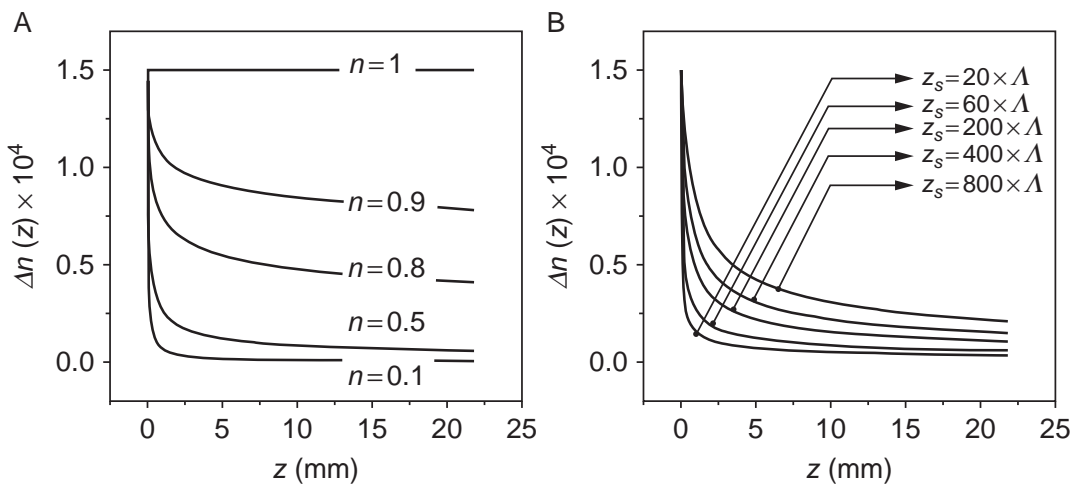

Fig. 32 (A) Different apodization profiles as a function of the grating position $z$ and with the fractional order of integration $n$ as a parameter, for a fixed $z_{s}=60 \times \Lambda$, with $\Lambda$ the grating period. (B) Same as before, but for varying $z_{s}$ and a fixed $n=0.5$. From CuadradoLaborde, C. \& Andrés, M. V. (2010). Proposal and design of an in-fiber all-optical fractional integrator. Optics Communications, 283, 5012-5015, https://doi.org/10.1016/j.optcom. 2010.07.040. 
number (see Fig. 1A). In this way, the first-order integrator proposed by Azaña (2008) could be considered a limiting case of this PFI. Fig. 32B shows the dependence of the apodization profile with the spatial shift $z_{s}$ for a fixed $n=0.5$. Having in mind the experimental realization of these gratings, we should comment that the fabrication of a PFI becomes increasingly difficult as both the fractional order $n$ and spatial shift $z_{s}$ approaches zero; while the realization of a PFI with $n>0.5$ and $z_{s}>60 \Lambda$ appeared readily feasible.

The spectral characteristics of the PFI were numerically obtained by using the fundamental matrix approach (Yamada \& Sakuda, 1987). The parameters used were a maximum operative time window $T=210 \mathrm{ps}$ and a time shift $\tau=0.3 \mathrm{ps}$. The selected $T$ and $\tau$ result in the same grating length $L$ and spatial shift $z_{s}$ used for the example shown in Fig. 32A. Additionally, and in order to fulfill the weak coupling regime, it also used the same maximum index modulation depth shown in the example of Fig. 32. Fig. 33A shows the reflectivity of the resulting grating and Fig. 33B shows the phase response, in which we can observe the necessary phase shift at the central frequency $n \times \pi=0.5 \times \pi=1.57 \mathrm{rad}$. The response of the ideal 0.5 th-order fractional integrator-i.e., $(-j 2 \pi \nu)^{-0.5}$ - overlaps the curves shown in Fig. 33, demonstrating that there is a high degree of resemblance between the proposed and ideal transfer functions.

The performance of this PFI was numerically simulated (see Fig. 34). In the first example, the input waveform was a first-order Hermite-Gaussian
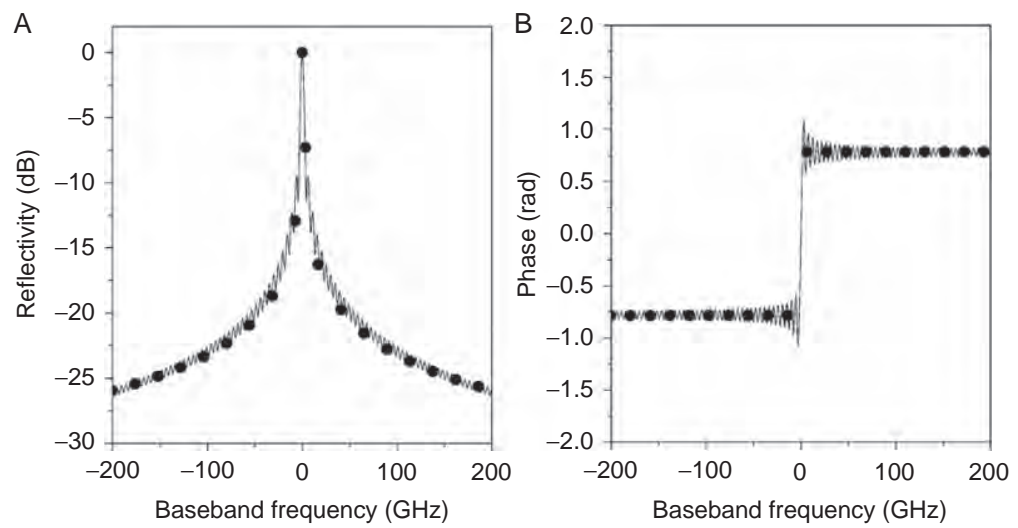

Fig. 33 Reflectivity and phase response of the proposed (solid curve) and ideal (i.e., theoretical, scatter points) 0.5 th-order fractional integrator $(A)$ and (B), respectively, in baseband frequency. From Cuadrado-Laborde, C. \& Andrés, M. V. (2010). Proposal and design of an in-fiber all-optical fractional integrator. Optics Communications, 283, 5012-5015, https://doi.org/10.1016/j.optcom.2010.07.040. 


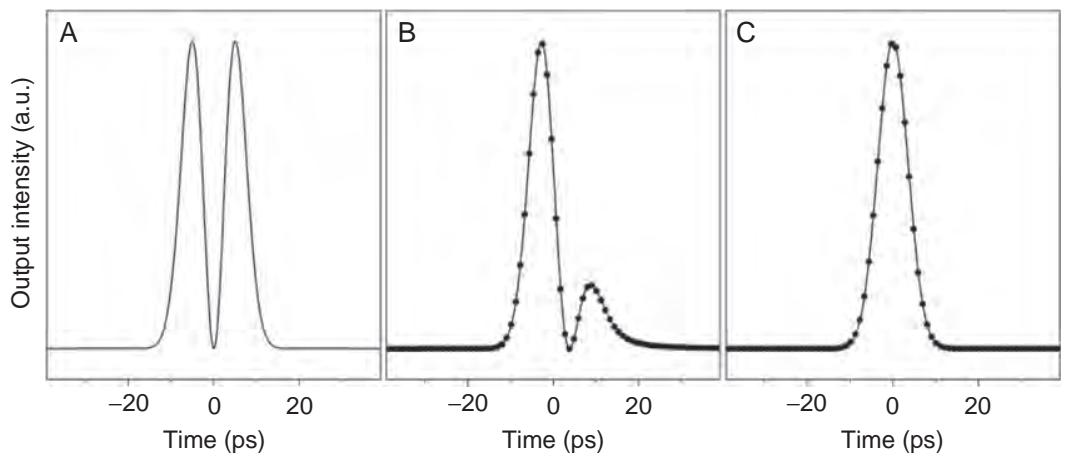

Fig. 34 (A) First-order Hermite-Gaussian pulse used as input signal ( $T_{0}=5 \mathrm{ps}$ ). (B) Output of the proposed 0.5th-order PFI (scatter points) and ideal time response (solid curve). (c) Same as before, but using as input the 0.5th-order PFI output shown in (B). From Cuadrado-Laborde, C. \& Andrés, M. V. (2010). Proposal and design of an in-fiber all-optical fractional integrator. Optics Communications, 283, 5012-5015, https://doi. org/10.1016/j.optcom.2010.07.040.

pulse $\left(\mathrm{HG}_{1}\right)$, numerically obtained through the first-order derivative of a Gaussian optical pulse, with a half-width (at $1 / e$ intensity point) $T_{0}=5 \mathrm{ps}$, i.e., a bandwidth of $54 \mathrm{GHz}$ (FWHM of the spectrum power) (see Fig. 34A). Fig. 34B shows the simulated temporal intensity at the output of the proposed 0.5 th-order PFI-whose reflectivity and phase response were shown in Fig. 33. For comparison, the ideal (analytical) 0.5th-order time integral is also shown; they are indistinguishable. Next, we test this 0.5 th-order PFI using as input signal the preceding output, i.e., the 0.5th-order fractional integral of a $\mathrm{HG}_{1}$ pulse, shown in Fig. 34B. The output after reflection in the 0.5 th-order PFI as well as its analytical counterpart is shown in Fig. 34C. As expected, this is equivalent to no transformation at all on the original Gaussian pulse, since two consecutive 0.5 th-order fractional integrals compensate the first-order derivative performed on the Gaussian pulse to obtain the $\mathrm{HG}_{1}$ pulse. Once again, we observe the accurate fractional time integration that is achieved. The viability of this proposal has not been tested experimentally to date.

\subsection{Uses and Applications of Photonics Fractional Calculus Operators}

Although a noticeable effort was done in the development of new photonic fractional-order differentiators with increasing capabilities (such as fractional-order tuning, operation bandwidth, etc.), much less progress was achieved demonstrating some advantage in the use of these devices 
for a specific task. The unfamiliarity with fractional tools by the scientific community could be among the reasons. However, there are a few exceptions which will be developed here.

As far as we know, the first step in this direction was given by CuadradoLaborde, Andrés, and Lancis (2011), when they theoretically discussed the possibility to use with some advantage the semidifferintegration (i.e., 0.5thorder fractional differentiation or integration) on the phase recovery of a given light pulse. A few years later, the same group successfully demonstrates experimentally the viability of their approach (Poveda-Wong et al., 2016c). On the other hand, this year, Liu et al. (2017) made one step further in this direction, when they proposed to perform high-speed PFD coding. These works will be described in Sections 2.4.1 and 2.4.2 with some details.

\subsubsection{Phase Recovery by Semidifferintegration}

The temporal phase profile of a light pulse (or its first time derivative, the instantaneous angular frequency) highly influences the performance in fiber-optic communication systems. For this reason, new solutions are demanded for monitoring of optical waveforms. Renowned techniques, such as the frequency-resolved optical gating (FROG) (Kane \& Trebino, 1993; Trebino, 2002), the spectral phase interferometry for direct electric field reconstruction (SPIDER) (Iaconis \& Walmsley, 1998), and the multiphoton intrapulse interference phase scan (MIIPS) (Lozovoy, Pastirk, \& Dantus, 2004) are of limited application for light pulses with time widths ranging from a few picoseconds to the nanoseconds regime. In the following, we describe a technique that makes use of either 0.5 th-order differentiation or 0.5 th-order integration to measure the instantaneous frequency of a light pulse in the tens of ps regime (Cuadrado-Laborde et al., 2011; Poveda-Wong et al., 2016c).

In the following, let us suppose a given optical pulse, whose complex temporal envelope is given by $g(t)=|g(t)| \exp [j \varphi(t)]$, with $j=\sqrt{-1}$. Let us suppose too, we have a photonic fractional differentiator, spectrally centered at zero baseband angular frequency. Now, we perform on this pulse not a standard spectrally centered, but a spectrally shifted 0.5 th-order fractional differentiation (with angular frequency shifting given by $\omega_{s}$ ). The signal that the PFD process can be written as $g(t) f(t)$, with $f(t)=\exp \left(j \omega_{s} t\right)$. Then, after some algebraic manipulations (whose details can be followed in the work of Cuadrado-Laborde et al., 2011), it can be demonstrated that the rate of change of the phase profile of the input pulse is related to the intensities 
of the original, and spectrally shifted semidifferentiation by the following approximation:

$$
\frac{\mathrm{d} \varphi(t)}{\mathrm{d} t} \approx \frac{\left|\frac{\mathrm{d}^{0.5}}{\mathrm{~d} t^{0.5}}\left[g(t) \exp \left(j \omega_{s} t\right)\right]\right|^{2}}{|g(t)|^{2}}-\omega_{s},
$$

In a similar way, if instead of a semidifferentiation, we apply to the input pulse a semiintegration, i.e., a 0.5 th-order fractional integration $\mathrm{d}^{-0.5} / \mathrm{d} t^{-0.5}$, also spectrally shifted by $f(t)=\exp \left(j \omega_{s} t\right)$, we obtain:

$$
\frac{\mathrm{d} \varphi(t)}{\mathrm{d} t} \approx \omega_{s}-\omega_{s}{ }^{2} \frac{\left|\frac{\mathrm{d}^{-0.5}\left[g(t) \exp \left(j \omega_{s} t\right)\right]}{\mathrm{d} t^{-0.5}}\right|^{2}}{|g(t)|^{2}},
$$

the mathematical details of Eq. (32) can be also followed in Appendix A of the work of Cuadrado-Laborde et al. (2011). Eqs. (31) and (32) show that the instantaneous frequency profile can be obtained by simply dividing two temporal intensity profiles: (i) the light pulse under test $|g(t)|^{2}$ and (ii) that of its corresponding spectrally shifted 0.5 th-order fractional differentiation or integration $\left|\mathrm{d}^{ \pm 0.5}\left[g(t) \exp \left(j \omega_{s} t\right)\right] / \mathrm{d} t^{ \pm 0.5}\right|^{2}$. The spectral shift $\omega_{s}$ should be high enough that the spectral content of the input pulse is mainly located at one side of the 0.5 th-order fractional differentiator resonance frequency. If required, the pulse's temporal phase profile can be obtained by numerical integration of Eq. (31) or (32), except by an undetermined numerical constant. It is worth noting that the noniterative nature of the proposed procedure; as opposed to other well-known techniques such as the Gerchberg-Saxton algorithm (Gerchberg \& Saxton, 1972), which precludes real-time applications. On the contrary, the technique proposed here is potentially well suited for real-time applications and nonrepetitive events.

Poveda-Wong et al. (2016c) provided experimental evidence for the first time of phase recovery by PFD of 0.5 th order. They performed PFD of 0.5 th order by using the LPG approach previously discussed (see Section 2.2.1 and Poveda-Wong et al., 2016b). The experimental setup used for PFD phase recovery is shown in Fig. 35. The light pulses under test - whose instantaneous frequency will be measured-were provided by a passively mode-locked ytterbium fiber laser, emitting at a fixed wavelength $\lambda_{0}=1038.5 \mathrm{~nm}$. The repetition rate of the mode-locked laser 


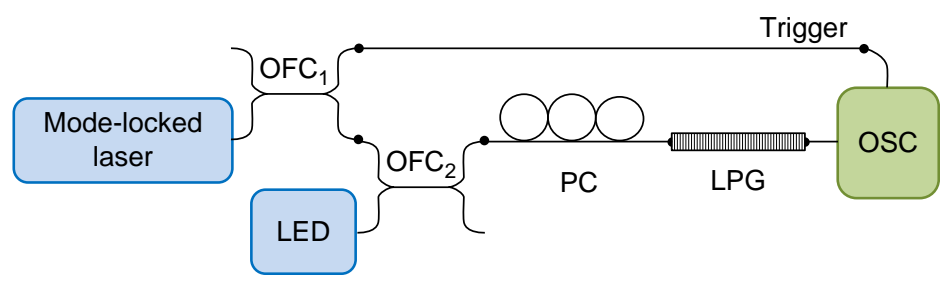

Fig. 35 Experimental setup used to measure the instantaneous angular frequency; where OFC, OSC, and PC stand for optical fiber coupler, oscilloscope, and polarization controller, respectively. The LPG is used to perform the 0.5 th-order fractional differentiation. From Poveda-Wong, L., Carrascosa, A., Cuadrado-Laborde, C., Cruz, J. L., Díez, A., \& Andrés, M. V. (2016c). Instantaneous frequency measurement by in-fiber 0.5 th order fractional differentiation. Optics Communications, 371, 89-92, https://doi.org/10.1016/ j.optcom.2016.03.061.

was of $23.15 \mathrm{MHz}$, and the output light pulses can be approximately fitted with an hyperbolic secant profile $g(t)=\operatorname{sech}\left(t / T_{0}\right)$, with $T_{0}=13 \mathrm{ps}$, i.e., a FWHM of 23 ps. This signal was split by a first optical fiber coupler (OFC1, 80/20); and one of its outputs (20\%) was sent to the trigger input of the oscilloscope through a $1.2 \mathrm{GHz}$ bandwidth photodetector (not shown). The second output port of the OFC1 was split by a second fiber-optic coupler (OFC2, 50/50) and propagated toward the LPG, where the state of polarization of the signal at the input of the LPG was previously adjusted through a PC. The temporal intensity waveform at the output of the LPG was detected with a $>63 \mathrm{GHz}$ sampling oscilloscope provided with a fast built-in photodetector $(53 \mathrm{GHz})$. Finally, a LED source in conjunction with an optical spectrum analyzer (replacing the OSC in Fig. 35) was used in those cases where the knowledge of the spectral position of the resonance transmission dip of the LPG was necessary.

The experimental procedure involved shifting in wavelength the transmission minima and maxima of the LPG-based PFD with respect to the operation wavelength of the mode-locked laser (which remains fixed). This allows the oscilloscope to register alternatively the intensity of the signal under test $|g(t)|^{2}$, or that of its corresponding spectrally shifted 0.5 th-order fractional differentiation $\left|\mathrm{d}^{0.5}\left[g(t) \exp \left(j \omega_{s} t\right)\right] / \mathrm{d} t^{0.5}\right|^{2}$. (This procedure can be followed in detail in the original work of Poveda-Wong et al., 2016c.) Fig. 36(left) shows both intensities as registered by the oscilloscope. The differences between both intensity profiles are due to the spectrally shifted fractional-order differentiation performed on the signal, since the trigger 

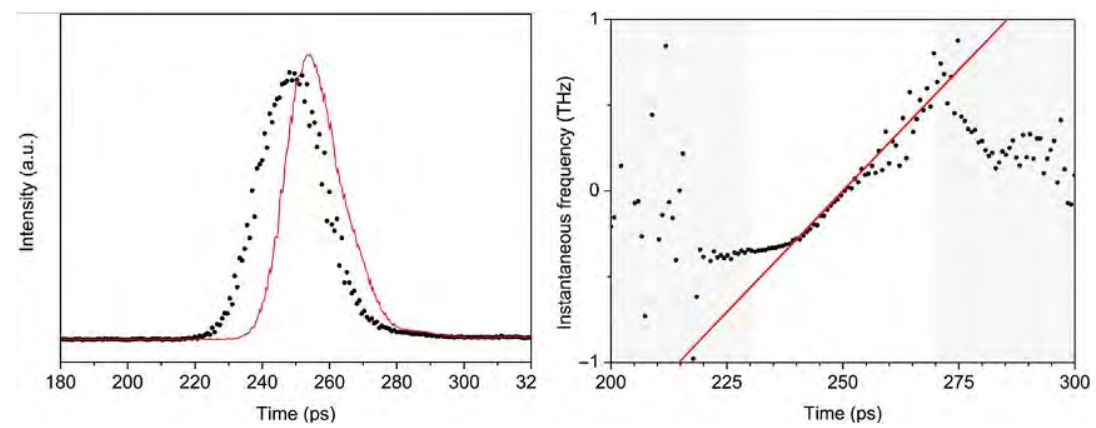

Fig. 36 (Left) Measured temporal intensity profiles of the optical pulse under test; and spectrally shifted 0.5 th-order fractional differentiation, scatter points and solid curve, respectively. (Right) Instantaneous frequency profile of the light pulse under test measured through in-fiber fractional-order differentiation, and linear instantaneous frequency profile corresponding to a chirp parameter $C=-30$, scatter points and solid curve, respectively. From Poveda-Wong, L., Carrascosa, A., Cuadrado-Laborde, C., Cruz, J. L., Díez, A., \& Andrés, M. V. (2016c). Instantaneous frequency measurement by in-fiber 0.5th order fractional differentiation. Optics Communications, 371, 89-92, https://doi.org/10.1016/j.optcom.2016.03.061.

signal used for the oscilloscope was the same in both cases; see the experimental setup in Fig. 35. The instantaneous angular frequency experimentally obtained is shown in Fig. 36(right), and it was obtained by dividing the temporal intensity profiles shown in Fig. 36(left), according to Eq. (31). It is worth mentioning that we should focus our attention to where the pulse energy is located; i.e., the unshaded central area in Fig. 36(right) which concentrates $95 \%$ of the input pulse energy. The measured instantaneous frequency profile of the input pulse $g(t)$ can be fitted linearly within the area of interest. This linearity for the instantaneous frequency necessarily implies a parabolic phase profile for the output pulse of the mode-locked laser $g(t)$, which could be described by $\exp \left(-j C t^{2} / 2 T_{0}^{2}\right)$, where $C$ is the chirp parameter, resulting in $C=-30$ according to our fitting. This result was further confirmed by the authors by independently measuring the instantaneous frequency by optical fiber dispersion technique (Cuadrado-Laborde et al., 2014).

The theoretical work of Cuadrado-Laborde et al. (2011) first, and the experimental confirmation of Poveda-Wong et al. (2016b) later, were the first experimental application, to the best of our knowledge, of a photonic device performing a fractional calculus operation in a specific problem, namely an instantaneous frequency profile measurement. 


\subsubsection{High-Speed Coding}

In Section 2.2.1, we described two different proposals of Liu et al. (2017) to perform a silicon-based integrated and tunable fractional-order PFD. As shown before (e.g., Fig. 29), a tunable PFD can shape a Gaussian pulse in different ways. Therefore, a high-speed coding system can be implemented based on such a differentiator, in which the coding can be done by applying a coding signal to PM1 (see Fig. 37(left)). Fig. 37(right) shows the coding map to represent a two-bit data sequence, a Gaussian pulse train can be differentiated with a fractional order of $0,0.5,1$, and 1.5 by applying a four-level data sequence to PM1.

Liu et al. (2017) experimentally validated their proposal by high-speed coding a Gaussian train of light pulses (see Fig. 38A); which in turn are sent to the active PFD. A synchronized four-level data sequence generated by an arbitrary waveform generator (AWG), as shown in Fig. 38B, is applied to PM1 in the differentiator (see Fig. 37). A coded pulse train is obtained at
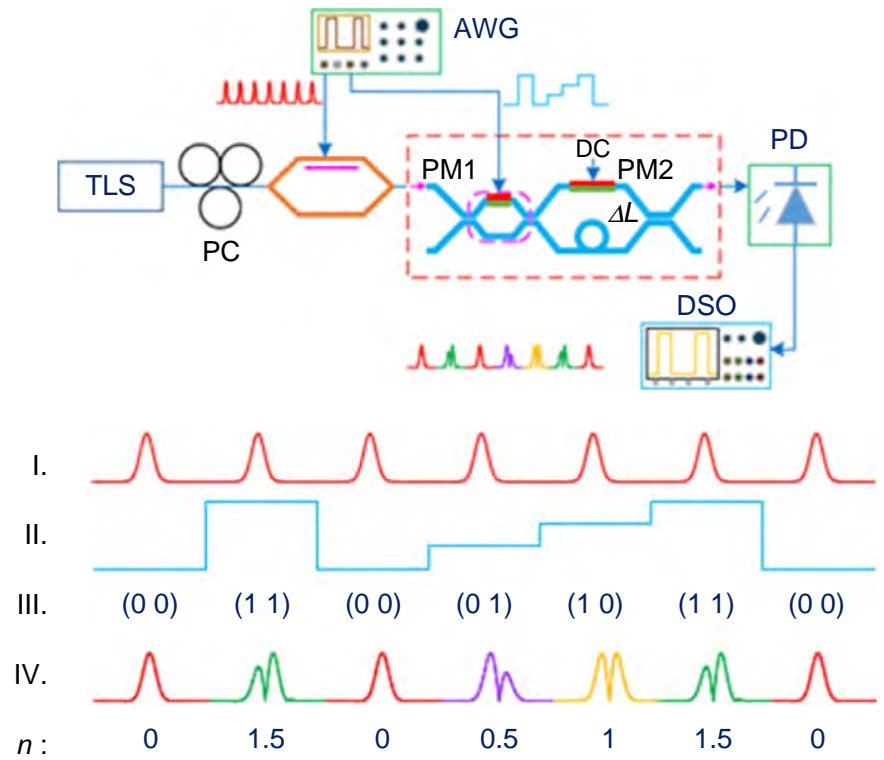

Fig. 37 (Left) Schematic of a high-speed coding system based on the active photonic temporal differentiator. (Right) The coding map of the proposed high-speed coding system with (I) a Gaussian pulse train, (II) a four-level data sequence, (III) a mapping two-bit data sequence, and (IV) the coded differentiation pulse train. From Liu, W., Zhang, W., \& Yao, J. (2017). Silicon-based integrated tunable fractional order photonic temporal differentiators. Journal of Lightwave Technology, 35, 2487-2493. https://doi.org/10. 1109/JLT.2017.2688468. 


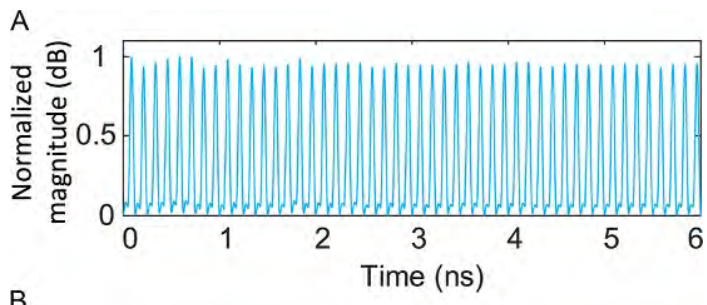

B

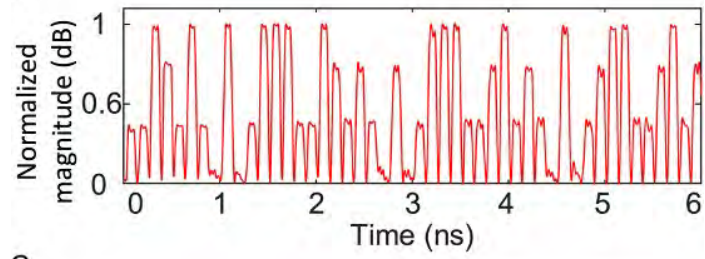

C
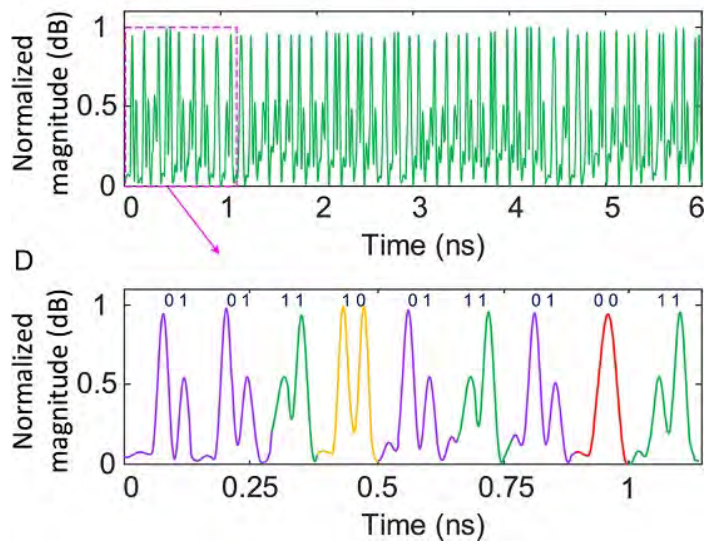

Fig. 38 Experimental results of high-speed coding based on the active photonic differentiator. (A) The Gaussian pulse train with a repetition rate of $8 \mathrm{GHz}$. (B) The synchronized four-level data sequence used for high-speed coding. (C) The generated differentiation pulse train with coded data. (D) A close view of the generated pulse train shown in (C). From Liu, W., Zhang, W., \& Yao, J. (2017). Silicon-based integrated tunable fractional order photonic temporal differentiators. Journal of Lightwave Technology, 35, 2487-2493. https://doi.org/10.1109/JLT.2017.2688468.

the output of the differentiator, as shown in Fig. 38C. A close view of the coded pulse train from in a $1 \mathrm{~ns}$ scale is also shown in Fig. 38D. As can be seen, the proposed active photonic temporal differentiator can be used for highspeed coding with a data rate of $16 \mathrm{Gbps}$. On the other hand, the decoding of the data sequence can only be successful by performing correlation between the encoded signal and a signature sequence with the coding map, thus this approach can find important applications in secure communications. 


\section{FRACTIONAL HILBERT TRANSFORM}

\subsection{Definition and Historical Overview}

The ordinary Hilbert transform (HT) $H\{$.$\} of a one-dimensional signal f(t)$ is defined by (Hahn, 2000):

$$
H\{f(t)\} \equiv \frac{1}{\pi} P \int_{-\infty}^{\infty} \frac{f(\eta)}{t-\eta} d \eta,
$$

where $P$ stands for the Cauchy principal value of the singular integral; since the integrand in Eq. (33) is improper because it goes to infinity as $\eta \rightarrow t$. While the Fourier transform flips the independent variable of a signal from the time to the frequency domain or vice versa, the HT leaves the signal in the same domain. The HT of a time signal is another time signal. It should be noted, that Eq. (33) can be written as a convolution:

$$
H\{f(t)\}=f(t) \circledast \theta(t),
$$

where $\theta(t)$ is the transform kernel in Eq. (33), whose definition and Fourier transform are given by:

$$
\theta(t)=1 /(\pi t) \stackrel{\mathfrak{I}}{\longrightarrow} \Theta(\omega)=-j \operatorname{sgn}(\omega),
$$

where $\operatorname{sgn}(\omega)$ is the signum function (which is +1 for $\omega>0$ and -1 otherwise), and $\omega$ is the baseband angular frequency, i.e., $\omega=\omega_{\text {opt }}-\omega_{0}$, where $\omega_{\text {opt }}$ is the optical frequency and $\omega_{0}$ is the central optical frequency of the signals. The operation given in Eq. (34), can be performed directly in the Fourier domain, given the well-known relationship between convolution in the time domain and multiplication in the Fourier domain:

$$
\mathfrak{J}[H\{f(t)\}]=\mathfrak{J}[f(t)] \Theta(\omega)
$$

Therefore, a HT can be performed by merely multiplying the input signal spectrum $\mathfrak{I}[f(t)]$ by the HT filter $\Theta(\omega)$. It is worth to emphasize that this HT filter has a $\pi$ phase shift at $\omega=0$, whereas the amplitude remains constant. Regarding its optical implementation, it can be easily implemented with a $4 f$ system with the insertion of a filter function located at $2 f$, consisting of a glass plate covered halfway by a $\pi$ phase shifting layer (Lohmann, Mendlovic, \& Zalevsky, 1998). This optical system was independently proposed by Kastler (1950) and Wolter (1950). The former proposed it for edge enhancement in image processing, since the HT 
emphasizes the derivatives of the image, whereas the later proposed it for spectroscopy applications.

The FHT $H_{n}\{$.$\} (where n \in R^{+}$) was proposed by Lohmann, Mendlovic, and Zalevsky (1996) as a generalization of the ordinary HT, and further analyzed by Davis, McNamara, and Cottrell (1998). They originally proposed two different-but nonequivalent - approaches (see Fig. 39), namely:

(A) preserving the conventional FT and fractionalizing the HT filter (see Fig. 39A), or

(B) preserving the conventional HT filter and fractionalizing the FT (i.e., by using the FFT) (see Fig. 39B).

Approach (A) can be performed by using the following fractional filter in the conventional Fourier domain:

$$
\Theta_{n}(\omega)=[-j \operatorname{sgn}(\omega)]^{n}
$$

This filter has a phase shift of $n \times \pi$ at $\omega=0$, and it also has a flat unitamplitude response, as its ordinary counterpart. In approach (A), the input signal $f(t)$ is Fourier transformed, then multiplied by the filter defined through Eq. (37), and finally inverse transformed to obtain the FHT of $f(t)$. In this approach, interestingly, it can be easily demonstrated also, that the FHT $H_{n}\{$.$\} is a mixture-weighted by n$-of the zeroth-order HT
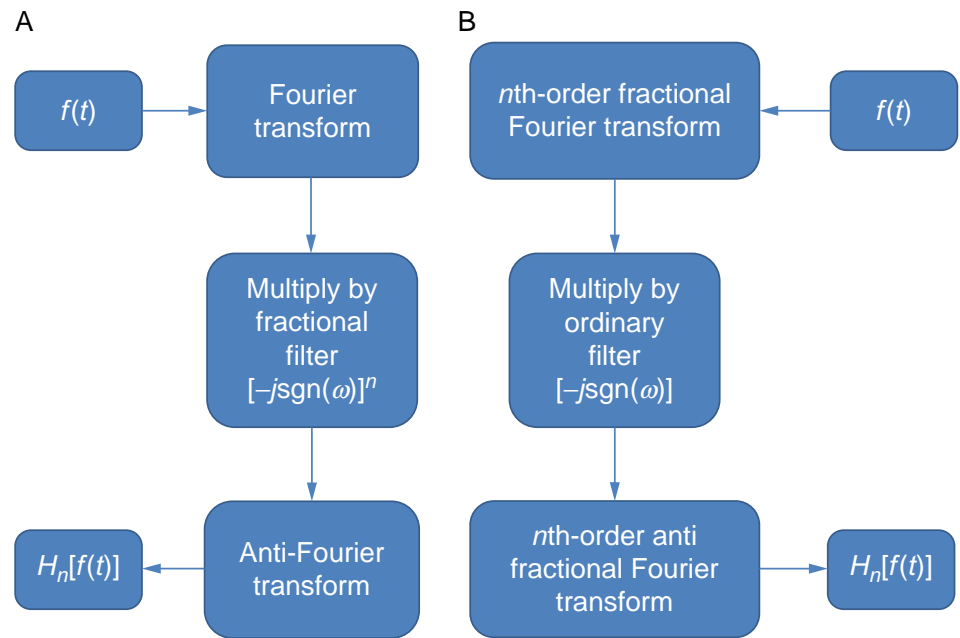

Fig. 39 Workflows for obtaining the fractional Hilbert transform according to Lohmann et al. (1996): (A) fractionalizing the ordinary Hilbert transform filter and (B) fractionalizing the Fourier transform and preserving the ordinary Hilbert transform filter. The approaches are not equivalents. 
$H_{0}\{f(t)\}=f(t)$-i.e., no transformation at all-and the first-order HT $H_{1}\{f(t)\}$ (Lohmann et al., 1996):

$$
H_{n}\{f(t)\}=\cos \left(\frac{n \pi}{2}\right) H_{0}\{f(t)\}+\sin \left(\frac{n \pi}{2}\right) H_{1}\{f(t)\} .
$$

Therefore, a FHT of arbitrary order could be designed also by properly combining the original input signal and the output of a first-order HT, according to Eq. (38). However, that approach could be experimentally challenging, because both signals should be carefully weighted and synchronized before they were combined. On the other hand, in approach (B), the input signal is fractionally Fourier transformed to the $n$th order, then multiplied by the ordinary HT filter $\Theta(\omega)$ defined by Eq. (37), and finally inversely transformed to the $-n$th FFT order (Lohmann et al., 1998).

Both approaches fulfill the reasonable postulate that the zeroth-order FHT reduces to the identity operation, whereas the first-order FHT should be consistent with the conventional HT. However, only the first definition is index additive, i.e., $H_{n}\left\{H_{m}\{\}.\right\}=H_{n+m}\{$.$\} . There remains$ also the possibility to perform both approaches simultaneously, something that can be reasonably named double-fractional HT. Neither approach (B) nor this doubly fractional possibility has been explored further, with the exception of the work of Lohmann et al. (1998). In this work, and from now on, we will refer exclusively to approach (A), i.e., by using the fractional filter given by Eq. (37) in the ordinary Fourier domain. On the other hand, and always in space optics, an experimental setup was proposed for approach (A), for one-dimensional (Lohmann, Ojeda-Castañeda, \& Diaz-Santana, 1996) and two-dimensional signals (Lohmann, Tepichín, \& Ramírez, 1997).

\subsection{Photonic Fractional Hilbert Transform}

In the following, we describe several approaches to PFHT. The proposals to be discussed have in common that they are all analog devices. There have been others proposals to perform PFHT with discrete multitap filtering too; e.g., the work of Li, Chi, Zhang, and Yao (2011). These works are out of the scope of this review and will not be analyzed in the following.

\subsubsection{The Bragg Grating Approach}

As far as we know, the first photonic analog fractional Hilbert transformer was proposed by Cuadrado-Laborde (2010). It was based on the in-fiber 
ordinary-order Hilbert transformer of Asghari and Azaña (2009). This in turn was designed by following the space-to-time mapping technique previously developed by Azaña and Chen (2002), which we succinctly develop in the following.

Any grating reflection response can be characterized by its frequency transfer function $H_{r}(\omega)=\left|H_{r}(\omega)\right| \exp \left[j \Phi_{r}(\omega)\right]$, with corresponding temporal impulse response $h_{r}(t)=\mathfrak{J}^{-1}\left[H_{r}(\omega)\right]$, where $\mathfrak{J}^{-1}$ denotes inverse Fourier transform. Kogelnik (1976) demonstrated that in the weak-grating limit; i.e., $\left|H_{r}(\omega)\right| \ll 1$ :

$$
A(z) \exp [j \varphi(z)] \propto h_{r}\left(t=\frac{2}{c} \int_{0}^{z} \mathrm{~d} z^{\prime} n_{a v}\left(z^{\prime}\right)\right)
$$

where $\propto$ represents proportionality, $c$ is the speed of light in vacuum, $A(z)$ describes the normalized apodization function (i.e., profile of the index modulation depth along the $z$-axis), $\varphi(z)$ represents the additional phase variation (chirp) in radians. In a few words, under the weak-coupling condition, the impulse response mimics the apodization profile in both, amplitude and phase; and vice versa. For a constant average refractive index $n_{a v}(z)=n_{a v}$, it follows immediately from Eq. (39) that

$$
\left.h_{r}(t) \propto\{A(z) \exp [j \varphi(z)]\}\right|_{z=c t /\left(2 n_{a v}\right)}
$$

for $0 \leq z \leq L$. Now, we can explain the procedure followed for a given FBG synthesis. This can be divided in three steps, which corresponds to the frequency, time, and space domains, respectively:

(i) A physically realizable transfer function should be defined.

(ii) The impulse response is obtained by Fourier antitransforming the equation obtained in the preceding step.

(iii) The apodization profile is obtained by using the time-to-space mapping in the equation obtained in the preceding step.

In this case, steps (i) and (ii) result in:

$$
h_{r}(t) \propto\left\{\mathfrak{I}^{-1}\left[H_{r}(\omega)\right] \Pi\left(\frac{t}{\Delta t}\right)\right\} \circledast \delta\left(t-\frac{\Delta t}{2}\right),
$$

where the real impulse response is obtained by Fourier antitransforming a physically realizable transfer function. This is the reason why the original transfer function for the ordinary order $\operatorname{HT~} H_{r}(\omega)=j \operatorname{sgn}(\omega)$ is multiplied by the box function $\Pi($.$) , in order to limit its bandwidth and time response$ duration by $\Delta t$. Finally, since it is necessary to ensure causality, the resulting 
function is time shifted through a convolution with the Dirac delta function. Next, step (iii) is followed by applying Eq. (41), resulting in (Asghari \& Azaña, 2009):

$$
\Delta n(z) \propto \frac{\sin ^{2}\left[\frac{n_{a v} \Delta \omega\left(z-z_{c}\right)}{2 c}\right]}{z-z_{c}},
$$

where $0 \leq z \leq L$, with $L=c \Delta t /\left(2 n_{\mathrm{av}}\right)$ the total grating length, $z_{c}=L / 2, n_{a v}$ is the average refractive index of the uniform period FBG, and $\Delta \omega$ is the required operative bandwidth. Eq. (42) leads to an apodization profile antisymmetrical with respect to the zero-crossing point $z_{c}$. It could be considered that this point divides two different gratings, each one with lengths $L_{1}$ and $L_{2}$, and $L=L_{1}+L_{2}$, one with positive-only index change and the other with a negative-only index change. When both gratings have an identical maximum index modulation depth $\Delta n_{p}{ }^{(i)}$ (with $i=1,2$ for the left and right gratings), the reflection phase shows a phase shift of $\pi$ at $\omega=0$, which is precisely the behavior required for implementing a first-order HT. However, Cuadrado-Laborde (2010) found that this phase shift can be continuously tuned up to a maximum value of $\pi$, by varying the relative maximum index modulation depths $\varepsilon_{\Delta n}=\Delta n_{p}{ }^{(2)} /\left|\Delta n_{p}{ }^{(1)}\right|$ at both sides of the zerocrossing point, without severely affecting the flatness in the amplitude response. Fig. 40A shows the resulting grating apodization profile.

The FBG was simulated using the transfer matrix approach (Yamada \& Sakuda, 1987). Fig. 40B shows the dependence of the fractional order $n$ of the FHT with the relative change of the index modulation depth $\varepsilon_{\Delta n}$ by keeping $\Delta n_{p}{ }^{(2)}=1.8 \times 10^{-4}$ fixed and varying $\Delta n_{p}{ }^{(1)}$, with a central operation wavelength of $1550 \mathrm{~nm}, n_{\mathrm{eff}}=1.452$, and $L_{1}=L_{2} \approx 10 \mathrm{~mm}$ - for simplicity, the length of each uniform FBG was fixed to be an integer

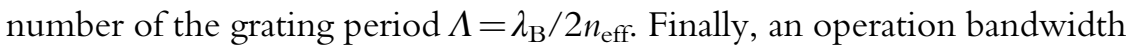
$\Delta \nu=150 \mathrm{GHz}$ was used. The author found that the phase shift-and consequently the FHT order $n$-only depends on the ratio between the maximum index modulation depths $\varepsilon_{\Delta n}$. On the other hand, the zero-crossing point $z_{c}$ should be located preferably at the middle of the grating; i.e., $L_{1}=L_{2}$ (see Fig. 40A). However, it is worth to mention that a few percent mismatch in the localization of $z_{c}$ does not have noticeable effects in the phase shift at $\omega=0$. Fig. 41A and B shows the field reflectivity and reflection phase, respectively, with same FBG parameters specified above and $\varepsilon_{\Delta n}=0.5$. As a consequence of using an asymmetrical FBG, the phase shift 

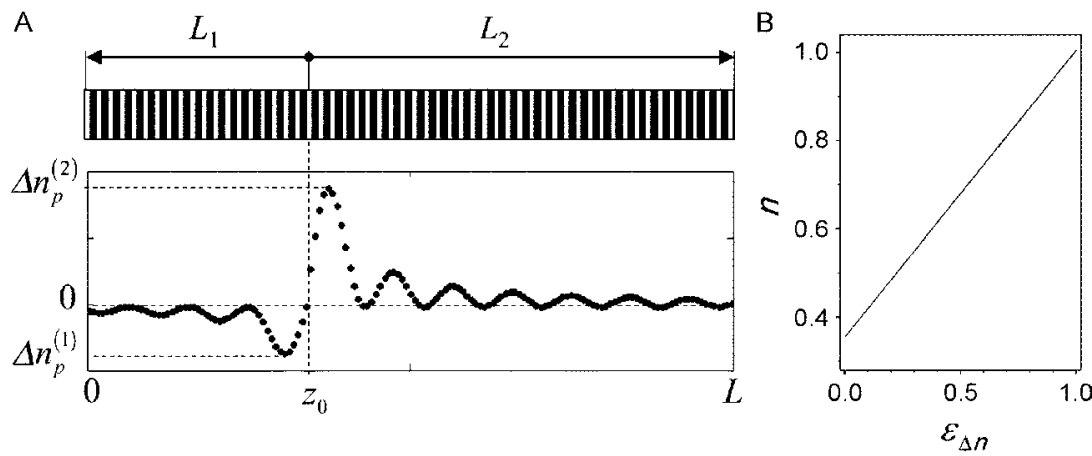

Fig. 40 (A) Above: Scheme of the FBG proposed for implementing a FHT; $L_{1}$ and $L_{2}$ are the grating lengths measured from the zero-crossing point. Below: Apodization profile over the fiber length $z, \Delta n_{p}{ }^{(1)}$ and $\Delta n_{p}{ }^{(2)}$ are the maximum index modulation depths at both sides of the zero-crossing point. (B) FHT order $n$ as a function of the relative change of the maximum index modulation depth $\varepsilon \Delta n$. From Cuadrado-Laborde, C. (2010). Proposal and design of a photonic in-fiber fractional Hilbert transformer. IEEE Photonics Technology Letters, 22, 33-35. https://doi.org/10.1109/LPT.2009.2035522 @ IEEE.
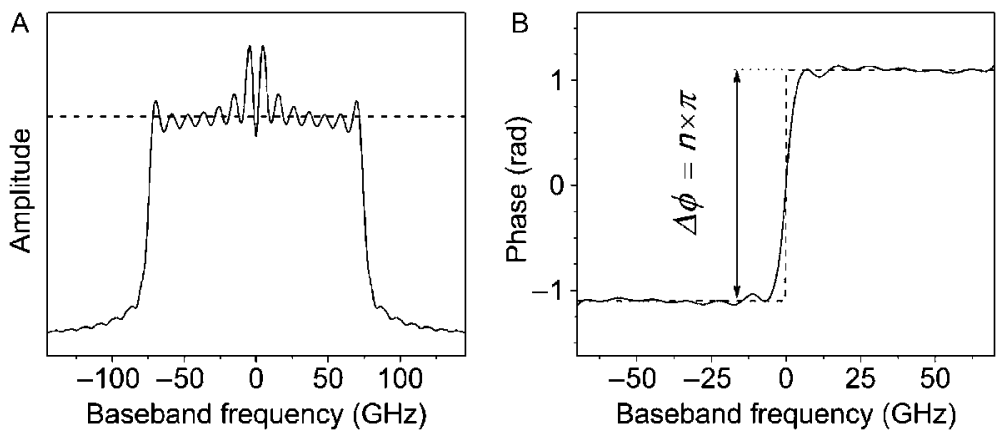

Fig. 41 Ideal (dashed curve) and proposed (solid curve) 0.7th-order FHT frequency response in (A) amplitude and (B) phase, in baseband frequency. Additionally, in (B) the average group delay was not represented, for illustration purposes. From Cuadrado-Laborde, C. (2010). Proposal and design of a photonic in-fiber fractional Hilbert transformer. IEEE Photonics Technology Letters, 22, 33-35. https://doi.org/10.1109/LPT. 2009.2035522 @ IEEE.

decreased to $\Delta \phi=2.2 \mathrm{rad}$ (see Fig. $41 \mathrm{~B}$ ), resulting in $n=\Delta \phi / \pi=0.7$. The ideal amplitude and phase response of the transfer function defined through Eq. (37) - with $n=0.7$ - is also shown, for comparison purposes. We observe that the phase response closely resembles the ideal behavior, except for the absence of a discontinuity at $\nu=0$, but showing a continuous phase change in a narrow spectral bandwidth (see Fig. 41B). 

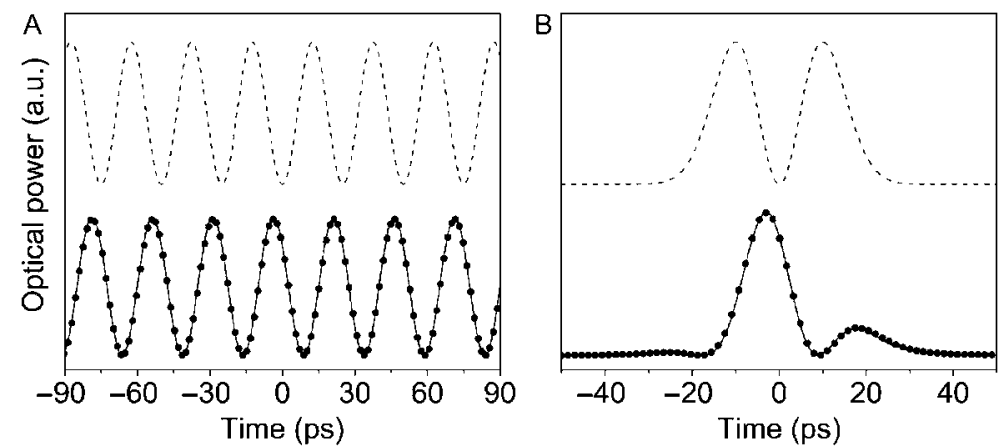

Fig. 42 Results from the numerical simulations when the input is: (A) a $20 \mathrm{GHz}$ sinusoid (dashed curve) and (B) a Hermite-Gaussian pulse (dashed curve). The time response of the designed 0.7th-order FHT is shown in each case (scatter points), together with the analytically obtained (solid curve). From Cuadrado-Laborde, C. (2010). Proposal and design of a photonic in-fiber fractional Hilbert transformer. IEEE Photonics Technology Letters, 22, 33-35. https://doi.org/10.1109/LPT.2009.2035522 @ IEEE.

The typical time domain response of this device was numerically simulated for a 0.7th-order FHT, whose amplitude and phase reflectivity are shown in Fig. 41. Fig. 42 shows the simulated temporal intensities at the output of the proposed FHT for two different test signals: a $20 \mathrm{GHz}$ sinusoidal signal and a first-order $\mathrm{HG}_{1}$ - obtained from the first derivative of a Gaussian pulse with a half-width (at $1 / e$ intensity point) of $T_{0}=10$ ps-see Fig. 42A and $\mathrm{B}$, respectively. For comparison purposes, the inputs and the ideal (analytical) 0.7 th-order FHT signals are also shown. There is a good agreement between the obtained time profiles and the theoretical (analytical) FHT of the input signals. A similar approach based on space-to-time mapping was also followed by Ge, Wang, and Zhu (2011), arriving at similar conclusions.

Along the same line, Liu et al. (2015) numerically analyzed the possibility to perform a wideband photonic fractional Hilbert transformer in a silica-on-silicon planar platform, by following Cuadrado-Laborde's (2010) approach. They numerically analyzed the effects of apodization profiles and operation bandwidths using a single apodized Bragg gratings. Fig. 43A-C illustrates the left-side halved apodization profile of the Bragg grating for PFHT, the grating amplitude response, and the grating phase response, respectively. The Bragg grating was set to $15 \mathrm{~mm}$ long, $n_{\mathrm{eff}}=1.4478$, and the operational bandwidth was $150 \mathrm{GHz}$. By reducing the maximum index modulation depth at one side of the zero-crossing point in the apodization profile to $\varepsilon_{\Delta n}=\Delta n_{p}{ }^{(2)} /\left|\Delta n_{p}{ }^{(1)}\right|=0.5$, a $0.73 \times \pi \mathrm{rad}$ phase shift was achieved in Fig. 43 C, i.e., a fractional order $n=0.73$. In Fig. $43 \mathrm{~B}$, it can be observed that 

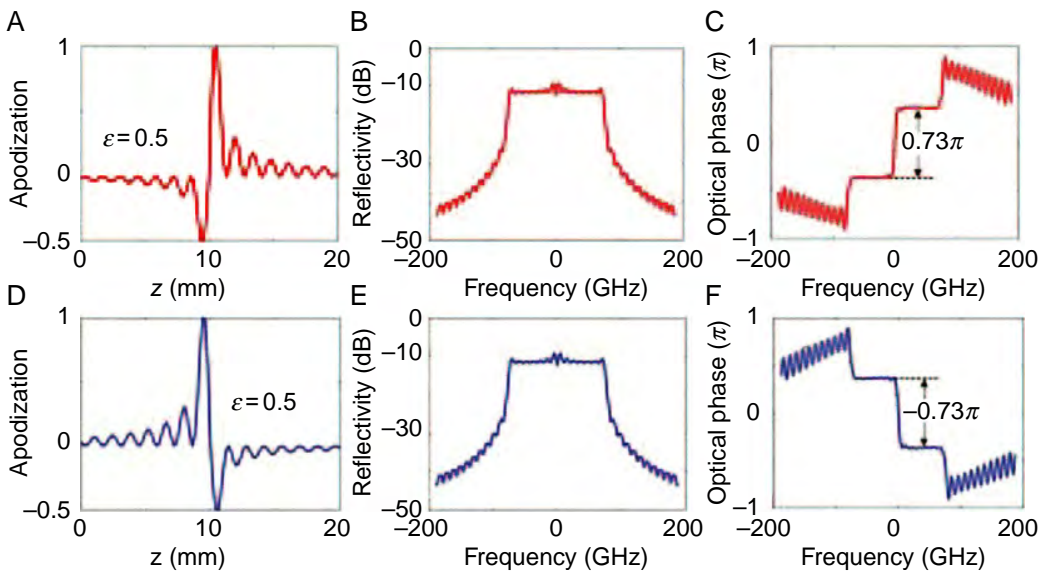

Fig. $43(\mathrm{~A}-\mathrm{C})$ The left-side halved grating apodization profile, the grating amplitude response, and the optical phase response (the average group delay was compensated for illustration purposes), $\varepsilon_{\Delta n}=0.5$ denotes the ratio of the maximum index modulation depths $\Delta n$ in the two sides of the zero-crossing point in the apodization profile; (D-F) the right-side halved apodization profile, as well as the grating amplitude and optical phase response. From Liu, B., Sima, C., Yang, W., Liu, D., Yu, Y., Gates, J., Zervas, M., Smith, P. (2015). Realization of Bragg grating based integrated fractional photonic Hilbert transformer. 11th conference on lasers and electro-optics pacific rim (CLEO-PR), 1-2. https://doi.org/10.1109/CLEOPR.2015.7376550.

the flat reflectivity required for PFHT is preserved. Interestingly, these authors also addressed the question of the inversion in the grating apodization profile (see Fig. 43D). In this case it can be observed that the amplitude response remains identical, while the phase shift is reversed to the same degree; i.e., $0.73 \times \pi$ rad phase shift (see Fig. $43 \mathrm{E}$ and F), respectively.

When changing the ratio $\varepsilon_{\Delta n}$, the factional order $n$ of the FHT will be consequently adjusted, and the relationship is plotted for direct and reversed apodization profiles in Fig. 44A and B, respectively. Although this numerical simulation was performed in a planar waveguide grating, the result is very similar to that previously shown in Cuadrado-Laborde's (2010) work for optical fiber gratings, as can be seen from comparing Figs. 40B and 44A. On the other hand, when implementing same $n$ th-order PFHTs, the bandwidth effect on the grating performance in terms of phase responses was investigated, as shown in Fig. 44C. Different color lines represent the optical phase responses of the PFHTs, with operating bandwidths increasing from 200 to $500 \mathrm{GHz}$. The data show that if operating bandwidths vary for proposed grating structures implementing PFHT, the fractional order $\rho$ is generally constant, e.g., $0.6 \pi$ in the plot. 

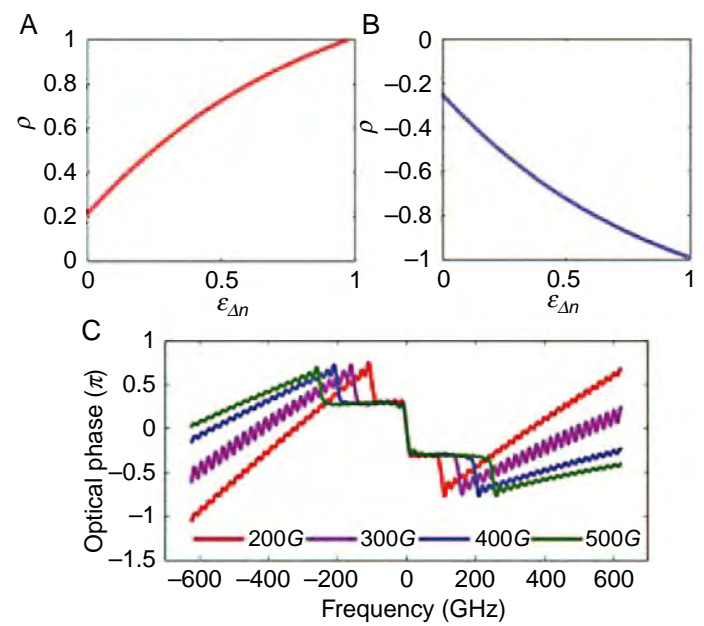

Fig. 44 (A) Fractional order $\rho$ as a function of the relative ratio $\varepsilon$ for the addictive phase shift; (B) $\rho$ as a function of $\varepsilon$ for the subtractive phase shift; (C) the grating phase responses implementing same order PFHT with different operating bandwidths. In the figures showing phase responses the average group delay was not represented for illustration purposes. From Liu, B., Sima, C., Yang, W., Liu, D., Yu, Y., Gates, J., Zervas, M., Smith, P. (2015). Realization of Bragg grating based integrated fractional photonic Hilbert transformer. 11th conference on lasers and electro-optics pacific rim (CLEO-PR), 1-2. https://doi.org/10.1109/CLEOPR.2015.7376550.

The viability of Cuadrado-Laborde's (2010) proposal for PFHT was experimentally demonstrated by Sima, Gates, Zervas, and Smith (2013a, 2013b), by using a planar Bragg grating-based approach. As an advantage compared with an FBG approach, the photonic bandwidth is clearly wider. Several PFHT with different characteristics were fabricated on the same substrate, using a direct UV grating writing technique (Emmerson et al., 2002; Sima et al., 2013a, 2013b), which is schematically shown in Fig. 45.

This method involves focusing two crossed laser beams $(\lambda=244 \mathrm{~nm})$ into the photosensitive core of a planar sample. Precise translation of the sample and modulation of the interference pattern creates grating structures and simultaneously defines the channel waveguide. The Bragg gratings sections were all $10 \mathrm{~mm}$ long, the effective refractive index of the mode within the gratings was 1.447. Fig. 46 shows the modeled data (blue line) and the measured data (red dashed line) of the grating amplitude response for Fig. 46A the 0.4 th-order FHT and Fig. 46B the 0.5th-order FHT. They also showed the potential of these structures to process THZ bandwidth signals. Fig. 47 shows the measurement in both reflectivity and group delay, of a PFHT of $1.5 \mathrm{THz}$ operative bandwidth $(12 \mathrm{~nm}$ at $1550 \mathrm{~nm})$. It can be observed the 


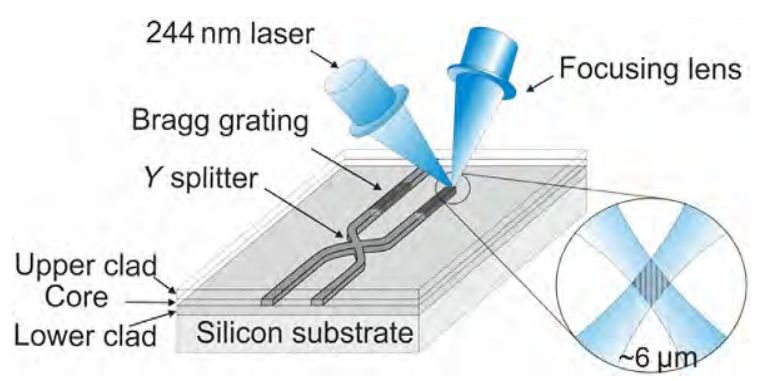

Fig. 45 Direct grating writing demo, showing crossed beams focused on the core layer of the silica-on-silicon substrate, with the writing spot in $\sim 6 \mu \mathrm{m}$ diameter. From Sima, $C$., Gates, J. C., Zervas, M. N. \& Smith, P. G. R. (2013a). Bragg grating based integrated photonic Hilbert transformers. 2013 IEEE international topical meeting on microwave photonics (MWP), Alexandria, VA, 96-99. https://doi.org/10.1109/MWP.2013.6724028.
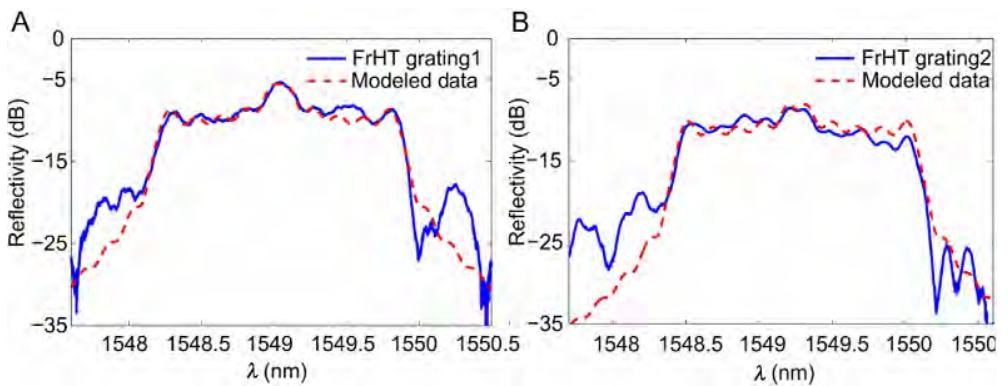

Fig. 46 Measured grating reflectivity spectra (blue line), with modeled data (red dashed line): (A) 0.4-order FHT and (B) 0.5-order FHT. From Sima, C., Gates, J. C., Zervas, M. N. \& Smith, P. G. R. (2013a). Bragg grating based integrated photonic Hilbert transformers. 2013 IEEE international topical meeting on microwave photonics (MWP), Alexandria, VA, 96-99. https://doi.org/10.1109/MWP.2013.6724028.
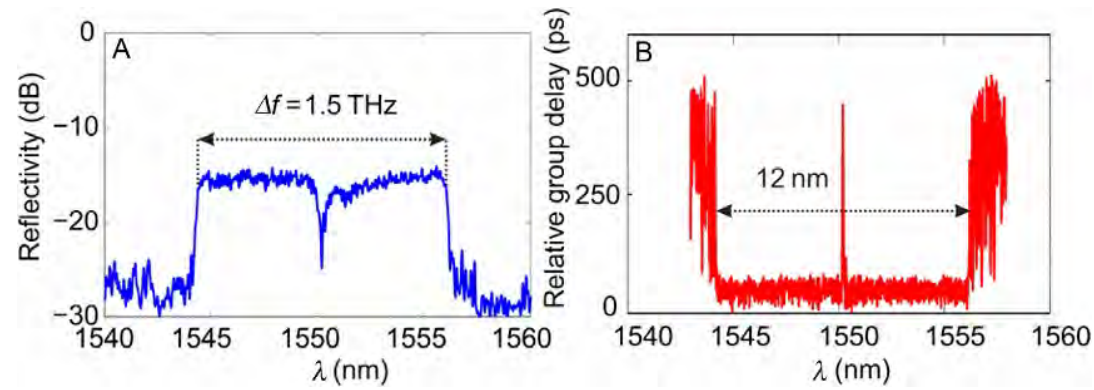

Fig. 47 (A) The measured reflectivity spectrum of the fabricated Bragg gratings implementing $1.5 \mathrm{THz}$ bandwidth PHT and (B) the measured relative group delay of the planar Bragg grating. From Sima, C., Gates, J. C., Zervas, M. N. \& Smith, P. G. R. (2013a). Bragg grating based integrated photonic Hilbert transformers. 2013 IEEE international topical meeting on microwave photonics (MWP), Alexandria, VA, 96-99. https:// doi.org/10.1109/MWP.2013.6724028. 
typical flat reflectivity required to perform a FHT, whereas in the time domain a delta peak is expected in the measurement of the group delay, which by temporal integration translates in a phase step discontinuity, as required.

Along the same line, Bazargani, Burla, Chrostowski, and Azaña (2016) experimentally demonstrate fractional-order photonic Hilbert transformation based on laterally apodized Bragg gratings in a SOI technology platform. The submillimeter-long gratings were fabricated using single-etch electron beam lithography, and the resulting HT devices offered operation bandwidths approaching the $\mathrm{THz}$ range, with time-bandwidth products between 10 and 20 .

Only 1 month after the original submission of Cuadrado-Laborde (2010), Li and Yao (2010a) also numerically investigated the possibility to perform an in-fiber PFHT. However, they followed a different direction. Their proposal relied on the discrete layer peeling (DLP) method. The FBG is directly designed based on the target response in the frequency domain by the DLP method. The key significance of using the DLP method over the approach followed by Cuadrado-Laborde (2010) for the FBG design is that the designed FBG is enabled to have a higher strength, improving the signal-to-noise ratio. The DLP method was previously developed by Feced, Zervas, and Muriel (1999), and subsequently improved by Skaar, Wang, and Erdogan (2001). Again, a physically realizable target spectrum must be defined approaching the desired one, in this case was defined by using a hyper Gaussian function of degree $m$, as follows:

$$
r(\omega)=R \exp \left[-\ln 2\left(\frac{2 \omega}{\Delta \omega}\right)^{m}\right] \exp [j \phi(\omega)]
$$

where $R$ is the reflectivity, $\Delta \omega$ is the operative bandwidth, and $\phi(\omega)$ is the required phase response (in this case $\phi(\omega)=n \pi / 2 \operatorname{sgn}(\omega)$ (see Eq. 37)), with $n$th order of the FHT. Fig. 48 shows the amplitude and phase responses of the ideal transfer function (Eq. 37) and the proposed transfer function (Eq. 43).

Fig. 49A shows the reflectivity and phase response of a 0.5 th-order FHT based on a $30 \mathrm{~mm}$ long multiple-phase-shifted uniform FBG; when $\Delta \omega=120 \mathrm{GHz}, R=0.9$, operative central wavelength of $1555 \mathrm{~nm}$, and $m=16$. The nonzero slope of the phase profile is a consequence of the causality condition. Fig. 49B shows the index modulation (left axis) and phase profile (right axis) of the FBG. The zero-to-zero width of the side lobes in 


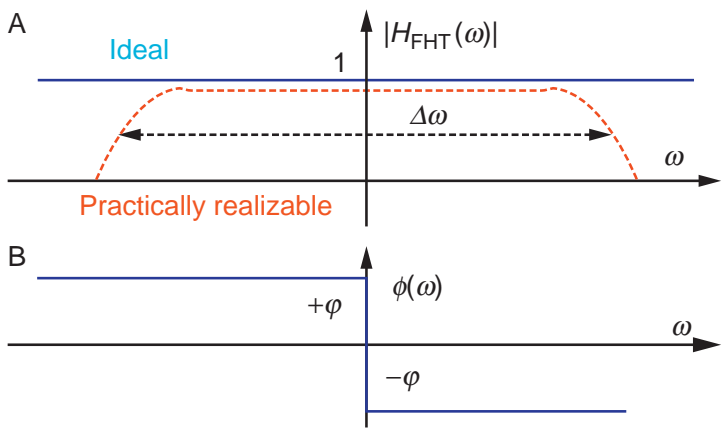

Fig. 48 Schematic diagram showing the (A) magnitude response and (B) phase response of an ideal (solid lines) and a practically realizable (dotted lines) photonic FHT. From Li, M. \& Yao, J. (2010a). All-fiber temporal photonic fractional Hilbert transformer based on a directly designed fiber Bragg grating. Optics Letters 35, 223-225. https://doi. org/10.1364/OL.35.000223.
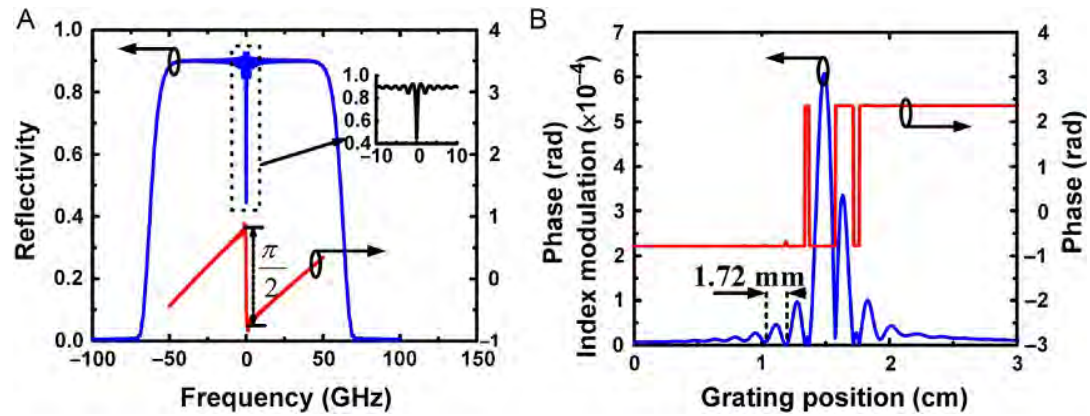

Fig. 49 (A) Magnitude and phase responses of the designed photonic FHT based on a $3 \mathrm{~cm}$ multiple phase-shifted FBG and (B) the index modulation and phase profile of the designed photonic FHT. From Li, M. \& Yao, J. (2010a). All-fiber temporal photonic fractional Hilbert transformer based on a directly designed fiber Bragg grating. Optics Letters 35, 223-225. https://doi.org/10.1364/OL.35.000223.

the apodization profile is about $1.72 \mathrm{~mm}$, which is determined by the bandwidth of the reflection spectrum. The maximum index modulation is about $6.07 \times 10^{-4}$. For a uniform FBG, a square-like response in the frequency domain corresponds to a cardinal sine index modulation profile. Therefore, the multiple phase shifts in the phase profile of the designed FBG are caused by the square-like 16th-order Gaussian magnitude spectrum. The multiple phase shifts are fabricated using a phase mask-based fabrication system by laterally shifting the phase mask with a high-precision piezoelectric positioning system to introduce the phase shifts (Cole, Loh, Laming, Zervas, \& Barcelos, 
1995; Loh, Cole, Zervas, Barcelos, \& Laming, 1995). Later, the viability of this proposal was experimentally demonstrated (Li \& Yao, 2010b). Unfortunately, $\mathrm{Li}$ and Yao only provided experimental results for the integerorder version of their approach, for two different operative bandwidths 50 and $100 \mathrm{GHz}$; the fractional performance was not experimentally tested in that work.

These Bragg gratings solutions to perform PFHT have in common that they are all designed to be used in reflection. Nevertheless, there are some reasons for using Bragg gratings in transmission. In this case, the complexity and cost of the system are reduced, since then couplers or circulators are avoided. In addition, the phase response in transmission is often more robust to imperfections in the grating structure than the phase response in reflection (Hinton, 1998). Unfortunately, a given complex filter function in transmission is both much more difficult to design and fabricate. Because the linear spectral response of transmissive Bragg gratings is necessarily minimum-phase, i.e., the phase spectral response of the grating transmission is uniquely related to its amplitude spectral response through a HT (Skaar, 2001). The minimum-phase is a very restrictive condition; indeed, the PFHT is a nonminimum-phase example; i.e., the phase and amplitude responses need to be tailored independently. One way to flip from a nonminimum-phase to a minimum-phase filter (physically realizable with a transmissive FBG) is creating a temporal impulse response that satisfies the minimum-phase condition by simply adding a temporal delta function before the target nonminimum-phase impulse response (advanced by a given time shift $\tau$ ). Using this technique, Fernández-Ruiz, Carballar, and Azaña (2014) theoretically first, and experimentally later (Fernández-Ruiz et al., 2015) proposed a transmissive FBG-based PFHT. Fig. 50 shows a typical example of the necessary apodization profile and periodicity of the FBG, obtained with this technique, in this case for an ordinary PHT.

Unfortunately, the output of this kind of PFHTs is a signal composed of two terms; one is a copy of the input, resulting from the convolution between the input and the delta function, and the second term is the desired Hilbert-transformed signal (Fernández-Ruiz et al., 2015). Thus, the delay time $\tau$ must be adjusted so that the two terms do not overlap. This imposes a necessary limitation on the maximum operation time window of the target processor. If needed, the copy of the input can be eliminated at the output by means of a time gating device. To summarize, in this technique, by using a FBG in transmission, optical circulators are avoided but at the price of obtaining an undesired signal at the output. 


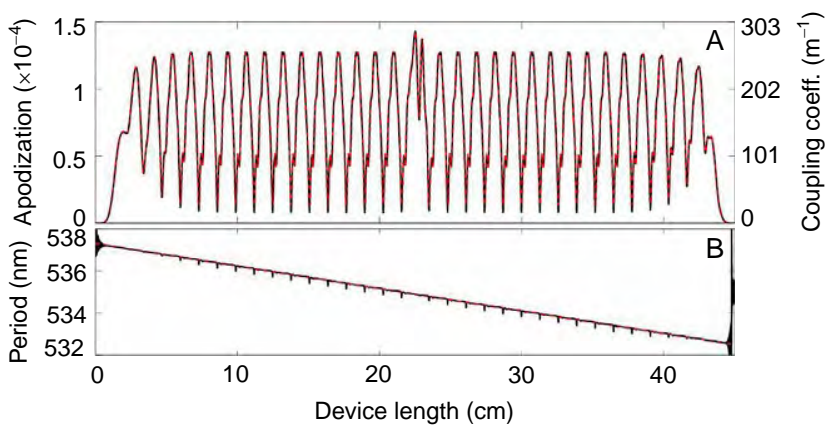

Fig. 50 PHT based on transmissive fiber BG: (A) Grating apodization profile: synthesized (black line) and with spatial resolution of $1 \mathrm{~mm}$ (dashed red line) and (B) period: synthesized (black line) and linearized assuming the prescribed chirp (dashed red line). From Fernández-Ruiz, M. R., Carballar, A., \& Azaña, J. (2014). Arbitrary time-limited optical pulse processors based on transmission Bragg gratings. IEEE Photonics Technology Letters, 26, 1754-1757. https://doi.org/10.1109/LPT.2014.2332102.

\subsubsection{The Fourier Domain Filtering Approach}

A completely different approach to PFHT was taken by Han, Li, Pan, Li, and Yao (2011). In contrast to the previously studied designs based on passive approaches, their device involves the use of active elements. Further, this was one of the first tunable PFHT; i.e., in this approach it is possible to select the desired fractional order, something that it is not possible with an already recorded grating (Cuadrado-Laborde, 2010). This technique uses a temporal pulse shaping system consisting of a PM and two dispersive elements with complementary dispersion. The PFHT is realized if a step function is applied to the PM to introduce a phase jump to the spectrum of the microwave signal to be Hilbert transformed. The tunable PFHT was implemented using the setup shown in Fig. 51.

The physical-mathematical principle is very simple, and can be summarized as follows (more details can be found in the original work of Han et al., 2011). The photonic setup works essentially like a $4 f$ system. First, there is a dispersive element (SMF in Fig. 51), which performs a simile of a Fourier transform. Next the PM carries out the necessary phase only discontinuity in the spectrum (see Eq. 37). Finally the second dispersive element (DCF) performs a second Fourier-like transform, returning the signal to the time domain. Regarding the Fourier transformers, both the SMF and the DCF (see Fig. 51), actually perform a Fraunhofer-like transformation. In intensity, there is no distinction between them; provided $\left|\Delta t^{2} / \Phi_{20}\right| \ll 1$, with $\Delta t$ the time duration of the input signal, and $\Phi_{20}=\beta_{20} L$, with $\beta_{20}$ the second 


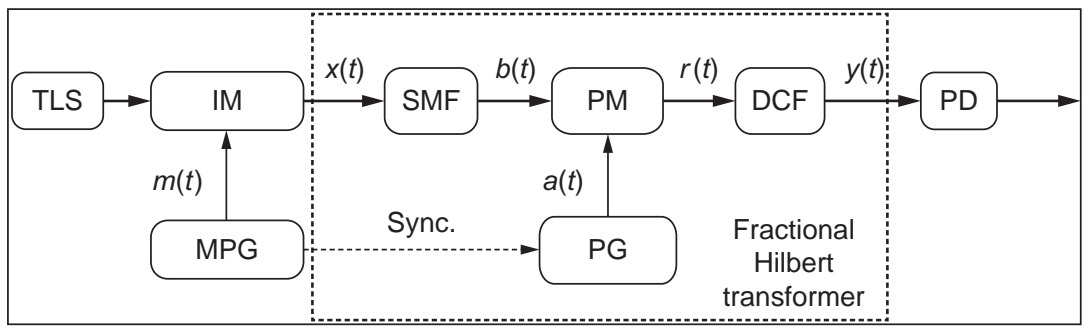

Fig. 51 Schematic of the temporal pulse shaping-based tunable PFHT system. TLS: tunable laser source; IM: intensity modulator; MPG: microwave pulse generator; SMF: singlemode fiber; $P M$ : phase modulator; $P G$ : pattern generator; $D C F$ : dispersion-compensating fiber; and PD: photodetector. From Han, Y., Li, Z., Pan, S., Li, M. \& Yao, J. (2011). Photonicassisted tunable microwave pulse fractional Hilbert transformer based on a temporal pulse shaping system. IEEE Photonics Technology Letters, 23, 570-572. https://doi.org/10.1109/ LPT.2011.2116113.

derivative of the propagation constant, and $L$ the fiber length (Muriel, Azaña, \& Carballar, 1999). However, the complex field at the output of the dispersive media is indeed affected by an additional linear chirp, which is inversely proportional to $\Phi_{20}$. The presence of the DCF with the same absolute dispersion as the SMF but with an opposite sign, reverses this effect. Here, the degree of phase discontinuity is regulated via the peak-to-peak voltage of the step voltage signal applied to the PM via the pattern generator. Han et al. (2011) experimentally demonstrated the viability of this proposal. In their experiment, a 550 fs temporal width light pulse provided by a modelocked laser was fractionally Hilbert transformed. The dispersive Fourier transformation (SMF and DCF, see Fig. 51) was performed by using two $36.91 \mathrm{~km}$ long optical fibers with total dispersions of $4872 \mathrm{ps}^{2}$ (SMF) and $-4872 \mathrm{ps}^{2}$ (DCF). The temporal width of the pulse at the output of the SMF was $4.9 \mathrm{~ns}$. A step function generated by an arbitrary waveform generator and amplified by a microwave amplifier is applied to the PM via the RF port. A fractionally Hilbert transformed pulse is thus obtained at the output of the DCF $y(t)$. However, since this pulse at the output of the DCF was back Fourier-transformed again, a light pulse of only a few picoseconds is expected, which is too fast to be detected by a photodetector. Therefore, in order to make the light pulses wide enough to be detected, they further stretched the fractional Hilbert transformed pulse $y(t)$ with a second SMF (not shown in Fig. 51) of $6.74 \mathrm{~km}$ long and dispersion of $-890 \mathrm{ps}^{2}$. The experimental results for the FHT with different fractional orders of $0.41,0.52$, and 0.71 are shown in Fig. 52. The simulated 


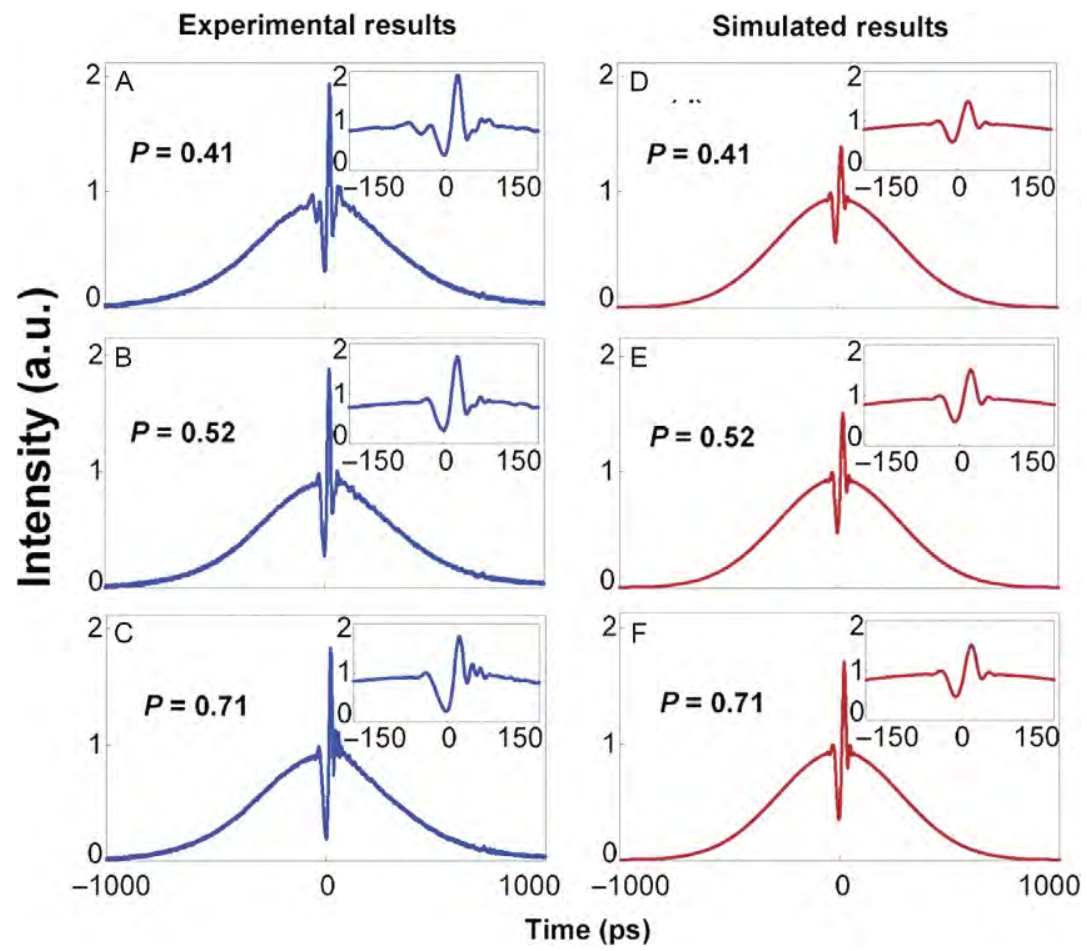

Fig. 52 Experimental results for the fractional orders of (A) 0.41, (B) 0.52, and (C) 0.71. The experimental results are compared with the simulation results in (D), (E), and (F). A zoom-in view of the central portion is given as an inset. From Han, Y., Li, Z., Pan, S., Li, M. \& Yao, J. (2011). Photonic-assisted tunable microwave pulse fractional Hilbert transformer based on a temporal pulse shaping system. IEEE Photonics Technology Letters, 23, 570-572. https://doi.org/10.1109/LPT.2011.2116113.

results - taking into account the additional stretching — are also shown. The experimental and simulated results agree reasonably well.

\subsubsection{The Long-Period Grating Approach}

When using FBGs in reflection, an arbitrarily specified spectral response in both amplitude and phase can be obtained through various inversescattering techniques, especially devoted to this purpose (Poladian, 2000). Unfortunately, following this approach implies the use of optical circulators; further, the operative bandwidths are limited to the tens of $\mathrm{GHz}$ regime, which is not enough for ultrashort applications. A broader bandwidth alternative to FBGs are LPGs that typically allow coupling of light from the fiber core mode into a copropagating cladding mode and thus operate inherently 
in transmission; therefore optical circulators can be omitted. However, to obtain a flexible filtering process in LPGs similar to that offered by FBGs, the input and output signals would need to be carried by different modes (e.g., input in the core mode and output in the cladding mode, or vice versa) (Feced \& Zervas, 2000). Owing to the difficulty to couple light in/out of a fiber cladding mode, Slavík, Kulishov, Park, and Azaña (2009) proposed an approach that overcomes this difficulty by employing two different concatenated LPFGs separated by a core-mode blocker (CMB). In this approach, one of the LPGs is designed with the required spectral response using a FBG-like inverse-scattering algorithm. If the light is originally in the core mode, then the output would be in the cladding mode. The CMB reduces parasitic interferences and can be performed by introducing between the LPGS a highly attenuating fiber section, or following the technique developed by Han, Kim, Lee, Paek, and Chung (2003). Once light in the cladding mode has been filtered as required, the second LPFG couples back the light to the core. Following this procedure, Ashrafi and Azaña (2012) theoretically proposed a PFHT device, whose scheme is shown in Fig. 53.

In Ashrafi and Azaña (2012) approach to PFHT, it is the first LPFG which performs the required response for a physically realizable PFHT, working in the core-to-cladding operation mode (see Fig. 53A). Next, light

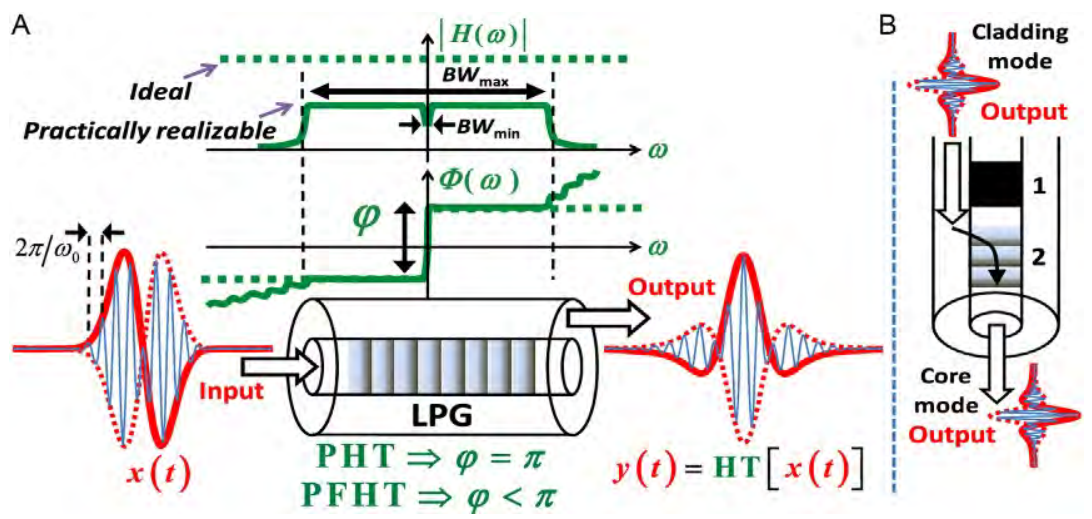

Fig. 53 (A) Schematic of the spectral amplitude $|H(\omega)|$ and phase $\Phi(\omega)$ responses of an ideal (green dotted lines) and a practically realizable (green solid lines) photonic fractional Hilbert transformer (output temporal curve corresponds to the ordinary first-order Hilbert transform. (B) The signal in the fiber cladding mode is transferred to the fiber core mode by using (1) a core-mode blocker and (2) a short, strong uniform LPG. From Ashrafi, R. \& Azaña, J. (2012). Terahertz bandwidth all-optical Hilbert transformers based on long-period gratings. Optics Letters, 37, 2604-2606. https://doi.org/10.1364/OL.37.002604. 

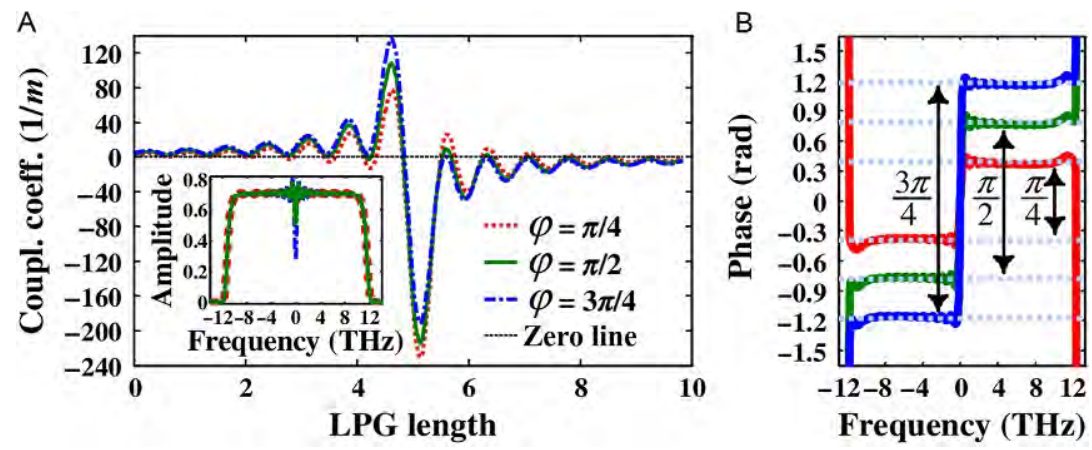

Fig. 54 (A) Designed apodization profiles for the LPG-based PFHTs with three different amounts of phase shifts in the spectral phase response: $\varphi=3 \pi / 4$ (dashed-dotted curve), $\varphi=\pi / 2$ (solid curve), and $\varphi=\pi / 4$ (dotted curve). The number of required $\pi$ phase shifts along the grating length for the cases of $\varphi=3 \pi / 4, \pi / 2$, and $\pi / 4$ are 3,7 , and 19, respectively. The corresponding simulated spectral phase and amplitude responses for each designed PFHT are shown in (B) and the inset of (A), respectively. From Ashrafi, R. \& Azaña, J. (2012). Terahertz bandwidth all-optical Hilbert transformers based on long-period gratings. Optics Letters, 37, 2604-2606. https://doi.org/10.1364/OL.37.002604.

propagates to the second LPG exclusively by the cladding (core-mode propagation is not allowed due to the presence of the $\mathrm{CMB}$ ), being coupled back to the core by a second broadband uniform LPG (see Fig. 53B). The LPGs are designed with a properly customized apodization profile, which is obtained by means of a discrete inverse-scattering algorithm (Brenne \& Skaar, 2003). Fig. 54A shows the obtained apodization profiles of the first LPGs for PFHTs with increasing fractional orders $n=1 / 4,1 / 2$, and $3 / 4$. The amplitude response is also shown in the same figure, whereas the simulated phase responses are shown in Fig. 54B. It is interesting to mention the broad bandwidth attainable with this technique, reaching up to tens of THz. As far as we know, this approach has not been experimentally validated to date.

\subsection{Present and Future Perspectives}

The topic of PFHT has been (together with PFD) one of the most actively pursued within the field of photonic fractional operations. The possibility to tune the fractional order was a great improvement which soon was implemented. Unfortunately, and as far as we know, it has not been demonstrated experimentally a specific advantage in the use of a PFHT to date. Our feeling is (as it happened before with PFD) that when more researchers know of this possibility, the applications will follow. As an example, the ordinary HT has been used recently for pulse characterization (Ahn, Park, \& 
Azana, 2007). On the other hand, Cuadrado-Laborde et al. (2010) demonstrated theoretically the potential of PFHT for the measurement of chirp in light pulses.

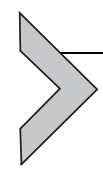

\section{FRACTIONAL FOURIER TRANSFORM}

\subsection{Definition and Historical Overview}

The FFT permits the representation of a temporal signal as a mixed time and frequency function, with a continuous degree of emphasis on time or on frequency features. It can be mathematically defined for a temporal signal $f(t)$ as (Lohmann, 1993; Ozaktas et al., 2001):

$F_{n}[f(t)]\left(t^{\prime}\right)=c(n) \int_{-\infty}^{\infty} f(t) \exp \left[\frac{j \pi\left(t^{2}+t^{\prime 2}\right)}{\varepsilon^{2}} \cot \left(\frac{n \pi}{2}\right)\right] \exp \left[-\frac{j 2 \pi t^{\prime} t}{\varepsilon^{2}} \csc \left(\frac{n \pi}{2}\right)\right] \mathrm{d} t$,

where $F_{n}[f(t)]\left(t^{\prime}\right)$ represents the FFT of $f(t), n$ is the fractional order, $t^{\prime}$ is the variable in the fractional domain, $c(n)=\varepsilon \exp (-j \pi / 4)[\csc (n \pi / 2)]^{1 / 2}$, and $\varepsilon$ is an arbitrary fixed parameter with time dimension. In Eq. (44), the reader can verify, by simple substitution, that the special cases $n=0$ and 1 are the trivial identity transform and the classical Fourier transform, respectively. The FFT is additive; i.e., performing two successive FFT of fractional order $n$ and $m$ on a given function, behaves identically to perform a single FFT of order $n+m$. It is worth emphasizing that the FFT belongs to a two-parameter family of transformations, namely: the fractional order $n$ and the scaling parameter $\varepsilon$. In an FFT, a change of the scaling parameter $\varepsilon$ thoroughly changes the functional form of the output; as opposed to the ordinary Fourier transform, where only a scaling in the reciprocal variable is accomplished (Lohmann, Zalevsky, Dorsch, \& Mendlovic, 1998).

Historically, and from a mathematical point of view, the idea of fractionalizing the Fourier transform dates back to 1929 (Bultheel \& Martínez, 2002; Torre, 2002). In the context of quantum mechanics it was rediscovered later by Namias (1980). In 1993, Mendlovic and Ozaktas (1993a, 1993b) demonstrated that the FFT of a given spatial light signal could be obtained through propagation in a graded index media. Ray propagation in such a medium can be shown to consist of periodically refocusing sinusoidal ray paths. One way of understanding this effect is to consider the GRIN 
medium as a series of thin lenses which gradually focus a diverging group of rays. Therefore, a piece of GRIN media of proper length $L$ is able to perform a two-dimensional Fourier transform at $z=L$, of the complex field $u(x, y)$ located at $z=0$. In this sense, if we cut-off a piece of GRIN media, reducing its length, for example, to $z=n L$, with $0<n<1$. The complex field at the exit of the shortened GRIN media is the $n$ th-order FFT. Also in 1993, two equivalent setups were proposed to perform an FFT by using bulk-optics components; i.e., a combination of lens action and free-space propagation (Lohmann, 1993; Lohmann et al., 1998). These setups were known as setups type I and II (see Fig. 55). Setup type I consists of free-space propagation, lens action, plus further free-space propagation, whereas setup type II consists of exactly the opposite; i.e., lens action, free space propagation, plus further lens action. Both, the focal length and the free space propagation length are tied to the FFT order by simple equations. The experimental demonstration of the FFT was performed with Lohmann's type I setup (Bitran, Mendlovic, Dorsch, Lohmann, \& Ozaktas, 1995). Although the GRIN approach could be useful for laboratory experiments, the superiority of the bulk-optics system is apparent because of its much higher space-bandwidth product performance and flexibility. Finally, Pellat-Finet (1994) first, and Hua, Liu, and Li (1997) later, realized that the Fresnel diffraction of a complex field under free-space propagation, is indeed a scaled FFT in intensity

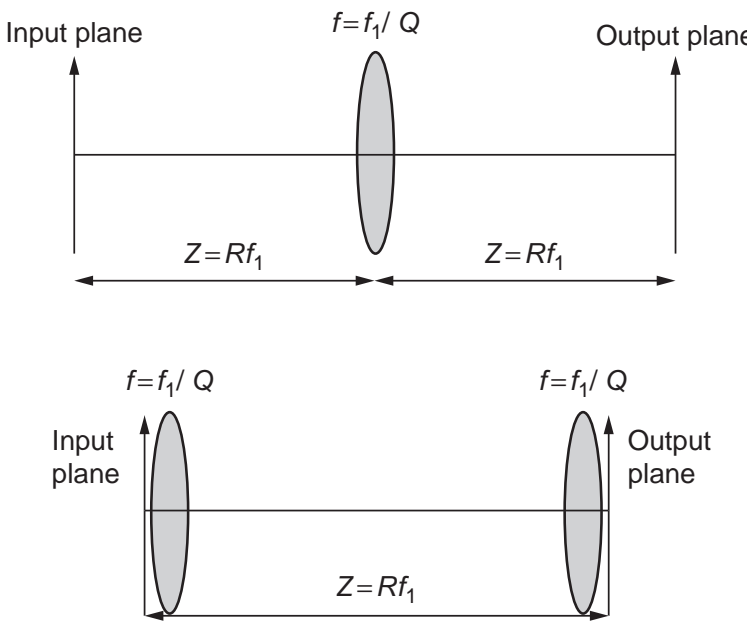

Fig. 55 Setups I (top) and II (bottom) to perform optically a FFT, with $f_{1}$ an arbitrary focal length, $R=\tan (\phi / 2)$ and $Q=\sin (\phi)$ (for setup I), or $Q=\tan (\phi / 2)$ and $R=\sin (\phi)$ (for setup II). In both cases $\phi=p \pi / 2$. 
(but not so in phase). Therefore, the intensity of a FFT can be obtained by simple free-space propagation in the near field regime.

\subsection{Photonic Fractional Fourier Transform}

\subsubsection{The Lohmann Approach}

The space-time duality for optical processing refers to the parallels between the paraxial diffraction of beams through space and the dispersion of narrowband pulses through dielectric media in time (van Howe \& Xu, 2006). Simply stated, a diffractive optical element such as a lens has a temporal analog that performs a parallel function in the time domain, namely an electro-optic PM. A complete table of equivalences can be found in table I of the work of van Howe and Xu (2006). The first proposal to perform a PFFT was provided by Lohmann and Mendlovic (1994), by translating to the time domain - via this space-time analogy - the setup type II proposed earlier by Lohmann to perform optically a FFT. As far as we know, this proposal was not performed experimentally, but theoretically discussed. Therefore, the type II tandem to perform a FFT in the space domain transformed, via space-time analogy, to the tandem chirp phase modulation-optical fiber propagation-chirp phase modulation. The quadratic phase modulation factor $\phi_{20}$ and first-order dispersion factor $\Phi^{\prime \prime}{ }_{20}$ are given by (Cuadrado-Laborde, Costanzo-Caso, Duchowicz, \& Sicre, 2007):

$$
\phi_{20}=\frac{2 \pi}{\varepsilon^{2}} \tan \left(\frac{p \pi}{4}\right), \quad \Phi_{20}=\frac{\varepsilon^{2}}{2 \pi} \sin \left(\frac{p \pi}{2}\right) .
$$

If we are only interested in the intensity of the FFT, then the use of the final quadratic PM can be avoided, since it is only required for correcting the phase term of the FFT (see Fig. 56).

Nevertheless, one PM is required at the input when using the approach depicted in Fig. 56. As far as we know, this setup has not been tested experimentally. One good reason for that is related with the cumbersome operation of the PM used as a time lens; we will come back to this point later. The first experimental demonstration of the possibility to perform a PFFT was provided by Cuadrado-Laborde, Carrascosa, Díez, Cruz, and Andres (2013). In this approach, a scaled version of the FFT is obtained in intensity of an arbitrary temporal light pulse, which is enough for several applications. We describe this approach in detail in the following. 


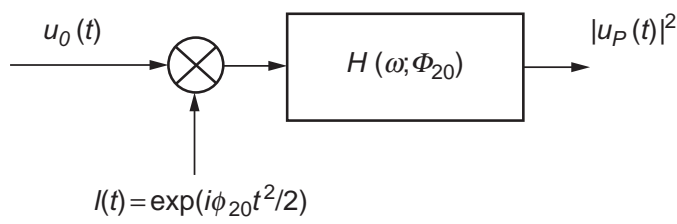

Fig. 56 Scheme of the optical device proposed to perform a PFFT. The input signal $u_{0}(t)$ is first phase modulated by $I(t)$ and then it is transmitted by the dispersive component having the transfer function $H(\omega)=\exp \left(i \Phi_{20} \omega^{2} / 2\right)$. The output optical power is $\left|u_{p}(t)\right|^{2}$. From Cuadrado-Laborde, C., Costanzo-Caso, P., Duchowicz, R., \& Sicre, E. E. (2007). Periodic pulse train conformation based on the temporal Radon-Wigner transform. Optics Communications, 275, 94-103. https://doi.org/10.1016/j.optcom.2007.03.021.

\subsubsection{The Fresnel Approach}

A linear dispersive medium can be modeled as linear time invariant systems by means of a transfer function. Let this transfer function $H(\omega)$ have unitary amplitude and quadratic phase response (i.e., linear group delay) over a certain spectral bandwidth $\Delta \omega_{H}$ :

$$
H(\omega)=\exp \left[-j \Phi_{20} \omega^{2} / 2\right],
$$

where $\omega$ is the baseband angular frequency and $\Phi_{20}$ is the first-order dispersion coefficient. Additionally, and only for simplicity, the average time delay has been ignored. Its impulse response-for complex envelopes of pulses with bandwidths narrower than $\Delta \omega_{H}$-will be given by anti-Fourier transforming Eq. (46), i.e.,

$$
h(t)=\exp \left[j t^{2} / 2 \Phi_{20}\right] .
$$

Next, the propagation of a single pulse in a linear dispersive regime can be expressed by convolving $f(t)$ with $h(t)$. Therefore, the output pulse $f_{o}(t)$ will be given by:

$$
f_{0}\left(t^{\prime}\right)=f\left(t^{\prime}\right) \circledast h\left(t^{\prime}\right) \propto \int_{-\infty}^{\infty} \mathrm{d} t f(t) \exp \left[j\left(t^{\prime}-t\right)^{2} / 2 \Phi_{20}\right] .
$$

Intensities in Eqs. (44) and (48) can be made equal, provided:

$$
\Phi_{20}=\left(\varepsilon^{2} / 2 \pi\right) \tan (p \pi / 2), \quad t_{p} \rightarrow t^{\prime} \cos (p \pi / 2) .
$$

Thus, the output of a linear dispersive system inherently provides a temporally magnified version in intensity of the FFT. This result can be considered as the time-domain counterpart of the existing equivalence between 
Fresnel diffraction and FFT, which has been shown before for the spatial case (Hua et al., 1997; Ozaktas, Arık, \& Coşkun, 2011).

Eq. (48) describes the propagation of temporal pulses, provided higherorder dispersion terms are negligible within the input pulse's bandwidth, including narrowband pulse propagation through an optical fiber. In that case:

$$
\Phi_{20}=z \beta_{20}, \quad D=-2 \pi c \beta_{20} / \lambda^{2},
$$

where $z$ is the fiber length, $\beta_{20}$ represents the second-order derivative of the propagation constant with respect to the angular frequency $\omega$, and $D$ is the dispersion parameter. Another solution widely used to provide enough dispersion is by using linearly chirped FBGs, because they can be designed with flat reflectivity and linear group delay within the operational bandwidth. Eq. (48) also describes the impulse response of linearly chirped FBGs; so the reflection of a pulse from a linearly chirped FBG is mathematically identical to Fresnel diffraction.

Cuadrado-Laborde et al. (2013) experimentally demonstrated the feasibility of this proposal by obtaining all-optically the FFT at different fractional orders on an input light pulse, by using different lengths of a standard optical fiber as a dispersive device. The input light pulses were provided by a passively mode-locked ytterbium fiber laser (emission wavelength of $1038 \mathrm{~nm}$ ), which can be satisfactory adjusted with a hyperbolic secant profile. By previous measurements, we also determined that the light pulses provided by this laser are linearly chirped with $C=-11$. In this way, the input light pulse was modeled by $f(t)=\operatorname{sech}\left(t / T_{0}\right) \exp \left(-j C t^{2} / 2 T_{0}^{2}\right)$, with $T_{0}=12 \mathrm{ps}$, which corresponds to a FWHM of 21 ps. The light pulses were detected with a $50 \mathrm{GHz}$ bandwidth sampling oscilloscope. The dispersion line was made with a commercially available optical fiber (SM980, low numerical aperture Fibercore), with a measured first-order dispersion $D=-44 \mathrm{ps} / \mathrm{nm} \mathrm{km}$ at the lasing wavelength. Three different fiber lengths were used: 101, 214, and $315 \mathrm{~m}$; by using Eq. (50), this results in the following first-order dispersion coefficients $\Phi_{20}=2.54,5.4$, and $7.94 \mathrm{ps}^{2}$, respectively. Finally, by using Eq. (49) and by selecting $\varepsilon^{2}=500 \mathrm{ps}^{2}$, they obtained the following fractional orders $p=0.0203,0.043$, and 0.063 , for each fiber length, respectively.

The experimental setup is shown in Fig. 57A. Fig. 57B shows the experimentally detected input pulse together with its corresponding fitting by a hyperbolic secant profile. The same figure shows the mathematically obtained 

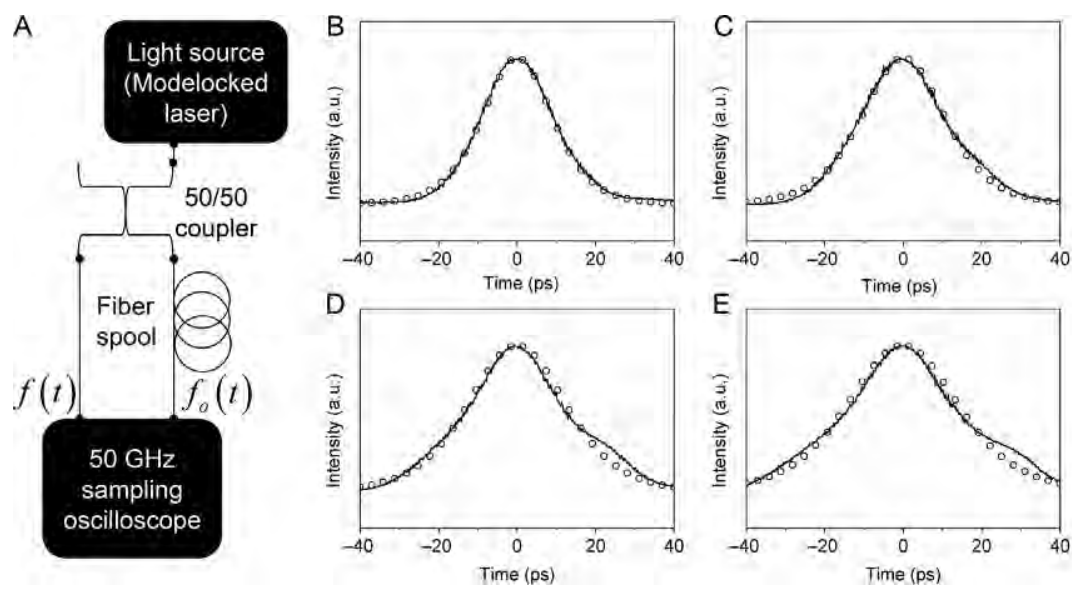

Fig. 57 (A) Scheme of the experimental setup. (B) Input pulse provided by the modelocked laser (solid curve), and its corresponding fitting by a sech profile (scatter points). (C) Mathematically obtained 0.0203 th-order FFT of the hyperbolic secant profile shown in (B), and experimentally detected output light pulse after propagation in a fiber length of $101 \mathrm{~m}$. (D and E) Same as in (C) but for 0.043th- and 0.063th-order FFTs and propagations of 214 and $315 \mathrm{~m}$, respectively. From Cuadrado-Laborde, C, Carrascosa, A., Díez, A., Cruz, J. L., \& Andres, M. V. (2013). Photonic fractional Fourier transformer with a single dispersive device. Optics Express, 21, 8558-8563. https://doi.org/10.1364/OE.21.008558.

FFT (scale factor $\varepsilon^{2}=500 \mathrm{ps}^{2}$ ) of the hyperbolic secant profile shown in Fig. 57B, for $p=0.0203$ (Fig. 57C), $p=0.043$ (Fig. 57D), and $p=0.063$ (Fig. 57E). For each theoretically obtained FFT, the experimentally detected output light pulse is also shown after propagating a fiber length of 101, 214, and $315 \mathrm{~m}$, respectively. The timescale of the propagated pulse was modified according to Eq. (49), with the corresponding fractional order. There is a good degree of resemblance between the analytically obtained and the experimentally detected FFT.

It is worthwhile to compare this proposal with the time-domain version of Lohmann's type I setup (Cuadrado-Laborde et al., 2007; Lohmann \& Mendlovic, 1994). In this case, it is necessary to use at least one PM with quadratic phase modulation factor of $\phi_{20}=1.11 \times 10^{20} \mathrm{rad} \mathrm{Hz}^{2}$; plus a LCFBG with a first-order dispersion coefficient of $\Phi^{\prime \prime}{ }_{20}=1.32 \times 10^{-21} \mathrm{~s}^{2}$ (see Eq. 45). Usually quadratic phase modulation is achieved by driving an electro-optic modulator with an electrical sinusoidal signal at the modulation frequency $\omega_{m}$-being $\phi_{20} \propto \omega_{m}{ }^{2}$ - which is only approximately quadratic at the cusp of the sinusoid. Therefore, the optical input signal must be restricted to a usable time aperture $T_{a}$ centered on the cusp. Thus, 
limiting the influence of higher-order (fourth) phase terms to about $2 \%$, results in $T_{a} \approx 1 / \sqrt{\phi_{20}}$. In this specific case, and by replacing by the aforementioned value, results in $T_{a} \approx 95$ ps. Not only the signal to be FFTed must be restricted to a maximum temporal width below $T_{a} \approx 95 \mathrm{ps}$, but also it should be adequately synchronized with the electrical signal feeding the electro-optic modulator. Therefore, the in-fiber FFT developed above is considerably simpler.

\subsubsection{The Talbot Approach}

Schnebelin and de Chatellus (2017) derived very recently an interesting relation based on the Talbot effect. They demonstrated that an $n$ th-order FFT of a given temporal signal can be computed simply by coherent addition of temporally delayed versions of the input signal itself after multiplication by a temporal chirp function. Next, these authors proposed an experimental setup enabling real-time FFT, providing numerical simulations to illustrate their concept. The technique involves two steps: a multiplication of the temporal signal under test by a chirp function, and the addition of regularly time-delayed versions of the resulting signal. Photonically, the multiplication by the chirp function is readily implemented by a quadratic phase modulation or by means of an acousto-optics frequency shifter driven by a linearly frequency modulated signal. The second step can be realized in an optical resonator (active or passive) or in an integrated multitap delay line (see Fig. 58B). Although it is somehow challenging to experimentally implement, it is fair to note that the order of the FFT can be simply tuned by changing the chirp rate and does not require adjusting the length of any delay line as in Cuadrado-Laborde et al.'s (2013) approach to PFFT.

\subsection{Uses and Applications of Photonic Fractional Fourier Transform}

The FFT has found widespread use, especially in signal processing. One of them is the optimal filtering in the fractional Fourier domain (Kutay, Ozaktas, Ankan, \& Onural, 1997). As a succinct introduction of the subject, it should be noted that there are signals with significant overlap in both the time and frequency domains. However, they could have little or no overlap at all in a fractional Fourier domain. To understand the underlying concept (see Fig. 59), where a Wigner (i.e., a simultaneous time-frequency representation) of a desired signal and an undesired noise term are superimposed in a given transmitted light signal. It can be easily 


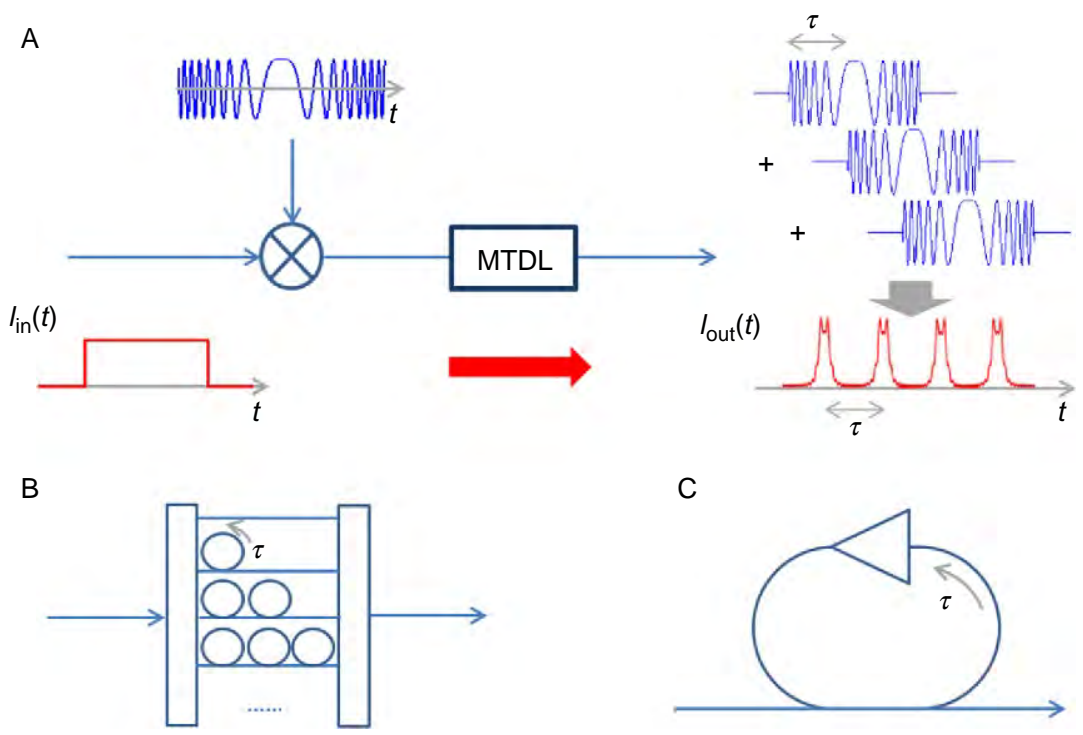

Fig. 58 (A) Principles of analog optical real-time FFT of an input top-hat signal (red) by multiplication by a chirp function and propagation through a multitap delay line (MTDL). The blue traces represent the real part of the signals, the red traces their intensity. (Bottom) Implementation of the MTDL in a multitap interferometer (B) and in a loop/ cavity (C). $\tau$ is the time delay between consecutive replicas of the signal. From Schnebelin, C. \& de Chatellus, H. G. (2017). Fractional Fourier transform-based description of the Talbot effect: application to analog signal processing. Applied Optics, 56, A62-A68. https://doi.org/10.1364/AO.56.000A62.

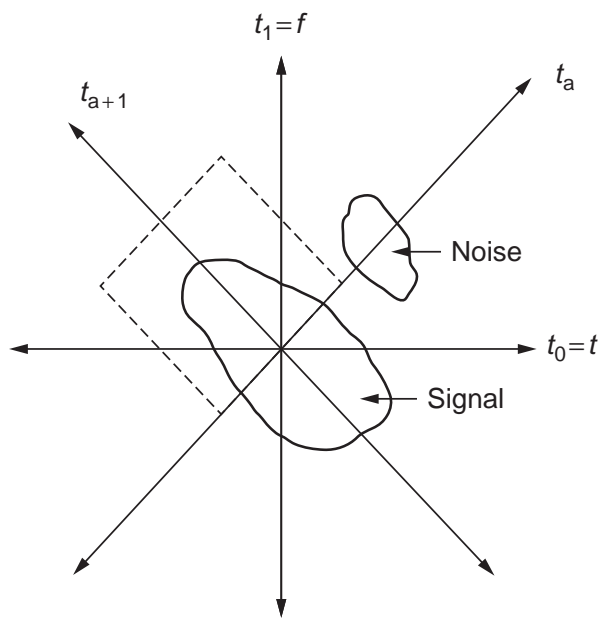

Fig. 59 Noise separation in the ath fractional Fourier domain. 
perceived they overlap (i.e., they project) on both time and/or frequency domains. However they do not overlap at all in the ath-order FFT domain. Thus, the undesired component can be easily removed by the following operations: (i) FFT of the compound signal to an optimal ath-order degree, (ii) multiply by a bandpass filter, and (iii) successive FFT of - ath-order (i.e., inverse FFT transform).

The FFT of a given signal is generally calculated numerically, involving a sequence of specific tasks: acquisition, sampling, and processing by a fast algorithm, which requires a large amount of computer resources. In contrast, analog computation does not make use of acquisition or processing systems and can be an interesting alternative to digital implementations. The sequence of operations described above for optimal filtering can be performed all-optically, which is especially interesting for real-time processing of noisy massive data. Very recently, Zhang et al. (2016) proposed an in-fiber system for real-time massive data processing in high-speed optical coherence tomography (OCT). OCT is a noninvasive in vivo imaging technique, which uses light waves to take cross-section pictures of tissue microstructure with a micrometer resolution. Although it was popularized by ophthalmologists in the analysis of retina, it has demonstrated a great utility in other applications also. In fact, for some specific applications such as surgical guidance, a real-time volumetric OCT is necessary. This action is necessarily performed in a four-dimensional space (4D-OCT). As an example, in real-time frequency domain high-definition OCT, it is necessary to perform a prohibitive number of fast Fourier transforms digitally. As an example 30 volumetric images per second of 1000 pixels along three axis results in $30 \times 10^{9}$ voxels/s. This is highly demanding for current electronic technology, even with the use of dedicated graphic processing units. However, in optical computing OCT, fast Fourier transformation of A-scan signal is optically processed in real time before being detected. Therefore, the processing time for interpolation and fast Fourier transform in traditional frequency domain OCT is eliminated. In the work of Zhang et al. (2016), they used in-fiber FFT to perform all-optically OCT at an impressive 10 M-A-scans/s, which represents a record for this technique.

On the other hand, Alieva, Bastiaans, and Stankovic (2003), derived a relationship between the instantaneous frequency (the signal's phase temporal derivative) and the derivative of the FFT intensity, with respect to the fractional-order parameter. This relationship is very similar to the wellknown transport-of-intensity equation for Fresnel diffraction. In a sense, this 
relationship is expected; given the close connection between Fresnel diffraction and FFT, which we explained before. Therefore, the instantaneous frequency, or the first derivative of the signal's phase, at any fractional domain is determined by the convolution of the angular derivative of the corresponding fractional power spectrum and the signum function. The in-fiber phase recovery technique proposed by Cuadrado-Laborde et al. (2014) can be considered as an example of this technique. However, since this technique relies on temporal intensity measurements, it is limited to phase recovery of picoseconds order light pulses. Brunel, Coetmellec, Lelek, and Louradour (2007) presented the first experimental demonstration of ultrashort pulse characterization by using fractional-order Fourier analysis on SPIDER-like interferograms (see also Coëtmellec, Brunel, Lebrun, \& Özkul, 2001). The FFT allows the determination of cubic spectral phase coefficients of ultrashort light pulses. Fractional-order Fourier series expansion has been demonstrated to allow a simultaneous determination of both cubic and quadratic spectral phase coefficients of the pulses. Although in this case the FFT was performed numerically, it could be performed all-optically in the future in order to improve the efficiency in realtime applications.

\section{CONCLUSIONS AND OUTLOOK}

Since the first works on photonic fractional operations (CuadradoLaborde, 2008; Cuadrado-Laborde \& Andrés, 2009), several interesting proposals were demonstrated in both fiber-optics and waveguide on-chip technologies. One key improvement, not originally contemplated in the first works, was the possibility to tune dynamically the fractional order; something which could be very advantageous in some specific circumstances (as we saw in PFD ultrafast coding, Section 2.4.2). However, these tuning proposals still need to be refined to avoid a dependence of the operation wavelength on the fractional order (which could represent a severe restraint), or a true full tuning range covering $0<n<1$ or even above 1 .

In the case of PFI, much less progress has been made as was reported above. Our experience tells us that some of the proposed first-order photonics integrators could work as a PFI, after some slight modifications. However, this work has not been done yet. Here it seems appropriate 
to mention the first-order photonic integrators developed by: Azaña (2008), Ferrera et al. (2011), Sun et al. (2016), Gopakumar and Madhusoodhanan (2017), and the references therein. One interesting application found for PFI (besides the phase recovery technique developed in Section 2.4.1) is in control systems, where the history of the system under control is important to the system output (Manabe, 1961). Takyar and Georgiou (2007) proposed a fractional integrator for set-point following in a control loop. Usually, the standard control design strategy for having zero steady-state tracking error requires a first-order integrator in the feedback loop. However, integer-order integrators add excessive lag, and this may adversely affect stability and robustness of the closed-loop system. In contrast, fractional integrators allow also for zero steady-state error in set-point following and, at the same time, introduce a smaller phase lag. In this way, an optical feedback control system could benefit from a PFI.

PFHT has received great attention too, and several alternatives have been proposed in both fiber-optics and waveguide on-chip technologies. Furthermore, some of these alternatives contemplate also the possibility to tune the fractional order. In this case, there has been a lack of experimental evidence of using the PFHT with some specific advantage. This is a direction which should be explored further.

Finally, perhaps the best known among these great unknown fractional operations, is the PFFT. Surprisingly, due to the several applications which have been found for the FFT, little progress has been made in this field with the exception of the works previously described. This is another field which should be explored further. As an example, and to the best of our knowledge, the well-known filtering in the fractional domain has not yet been explored for temporal light pulses.

\section{ACKNOWLEDGMENTS}

The authors apologize to all those whose publications may not have been mentioned properly in this chapter with a finite length. We acknowledge the Progress in Optics editor: Prof. T.D. Visser and his editorial team for the opportunity to contribute to this prestigious publication, together with their kind support throughout the whole editorial process.

This work has been financially supported by Agencia Estatal de Investigación (AEI) of Spain and Fondo Europeo de Desarrollo Regional (FEDER) (Ref.: TEC2016-76664-C2-1-R) and the Generalitat Valenciana of Spain (Ref.: PROMETEOII/2014/072). C.C.-L. acknowledges the financial support from project PICT 2008-1506 (ANPCyT, Argentina), PIP $11220150100607 \mathrm{CO}$ (CONICET, Argentina), and the Programa de Investigadores Invitados de la Universidad de Valencia (Spain). 


\section{REFERENCES}

Agrawal, G. P. (2001). Applications of nonlinear fiber optics. New York: Academic Press.

Ahn, T. J., Park, Y., \& Azana, J. (2007). In Pulse characterization using Hilbert transformation temporal interferometry (HTTI). Conference on lasers and electro-optics (CLEO), Baltimore, MD (pp. 1-2). https://doi.org/10.1109/CLEO.2007.4453311.

Alieva, T., Bastiaans, M. J., \& Stankovic, L. (2003). Signal reconstruction from two close fractional Fourier power spectra. IEEE Transactions on Signal Processing, 51, 112-123. https://doi.org/10.1109/TSP.2002.806593.

Asghari, M. H., \& Azaña, J. (2009). All-optical Hilbert transformer based on a single phaseshifted fiber Bragg grating: Design and analysis. Optics Letters, 34, 334-336. https://doi. org/10.1364/OL.34.000334.

Ashrafi, R., \& Azaña, J. (2012). Terahertz bandwidth all-optical Hilbert transformers based on long-period gratings. Optics Letters, 37, 2604-2606. https://doi.org/10.1364/OL.37. 002604 .

Azaña, J. (2008). Proposal of a uniform fiber Bragg grating as an ultrafast all-optical integrator. Optics Letters, 33, 4-6. https://doi.org/10.1364/OL.33.000004.

Azaña, J., \& Chen, L. R. (2002). Synthesis of temporal optical waveforms by fiber Bragg gratings: A new approach based on space-to-frequency-to-time mapping. Journal of the Optical Society of America B, 19, 2758-2769. https://doi.org/10.1364/JOSAB.19. 002758.

Bazargani, H. P., Burla, M., Chrostowski, L., \& Azaña, J. (2016). Photonic Hilbert transformers based on laterally apodized integrated waveguide Bragg gratings on a SOI wafer. Optics Letters, 41, 5039-5042. https://doi.org/10.1364/OL.41.005039.

Berger, N. K., Levit, B., Fischer, B., Kulishov, M., Plant, D. V., \& Azaña, J. (2007). Temporal differentiation of optical signals using a phase-shifted fiber Bragg grating. Optics Express, 15, 371-381. https://doi.org/10.1364/OE.15.000371.

Bitran, Y., Mendlovic, D., Dorsch, R. G., Lohmann, A. W., \& Ozaktas, H. M. (1995). Fractional Fourier transform: Simulations and experimental results. Applied Optics, 34, 1329-1332. https://doi.org/10.1364/AO.34.001329.

Brenne, J. K., \& Skaar, J. (2003). Design of grating-assisted codirectional couplers with discrete inverse-scattering algorithms. Journal of Lightwave Technology, 21, 254-263. https:// doi.org/10.1109/JLT.2003.808648.

Brunel, M., Coetmellec, S., Lelek, M., \& Louradour, F. (2007). Fractional-order Fourier analysis for ultrashort pulse characterization. Journal of the Optical Society of America A, 24, 1641-1646. https://doi.org/10.1364/JOSAA.24.001641.

Bultheel, A., \& Martínez, H. (2002). A shattered survey of fractional Fourier transform. http:// www.cs.kuleuven.ac.be/publicaties/rapporten/tw/TW337.pdf.

Coëtmellec, S., Brunel, M., Lebrun, D., \& Özkul, C. (2001). Characterization of chirped pulses with the fractional-order Fourier transformation. Journal of the Optical Society of America A, 18, 2754-2759. https://doi.org/10.1364/JOSAA.18.002754.

Cole, M. J., Loh, W. H., Laming, R. I., Zervas, M. N., \& Barcelos, S. (1995). Moving fibre/ phase mask-scanning beam technique for enhanced flexibility in producing fibre gratings with uniform phase mask. Electronics Letters, 31, 1488-1490. https://doi.org/10.1049/ el:19950986.

Cuadrado-Laborde, C. (2008). All-optical ultrafast fractional differentiator. Optical and Quantum Electronics, 40, 983-990. https://doi.org/10.1007/s11082-009-9282-5.

Cuadrado-Laborde, C. (2010). Proposal and design of a photonic in-fiber fractional Hilbert transformer. IEEE Photonics Technology Letters, 22, 33-35. https://doi.org/10.1109/LPT. 2009.2035522.

Cuadrado-Laborde, C., \& Andrés, M. V. (2009). In-fiber all-optical fractional differentiator. Optics Letters, 34, 833-835. https://doi.org/10.1364/OL.34.000833. 
Cuadrado-Laborde, C., \& Andrés, M. V. (2010). Proposal and design of an in-fiber all-optical fractional integrator. Optics Communications, 283, 5012-5015. https:// doi.org/10.1016/j. optcom.2010.07.040.

Cuadrado-Laborde, C., \& Andrés, M. V. (2011). Design of an ultra-broadband all-optical fractional differentiator with a long-period fiber grating. Optical and Quantum Electronics, 42, 571-576. https://doi.org/10.1007/s11082-011-9478-3.

Cuadrado-Laborde, C., Andrés, M. V., \& Lancis, J. (2011). Self-referenced phase reconstruction proposal of $\mathrm{GHz}$ bandwidth non-periodical optical pulses by in-fiber semidifferintegration. Optics Communications, 284, 5636-5640. https://doi.org/10.1016/j. optcom.2011.07.077.

Cuadrado-Laborde, C., Carrascosa, A., Díez, A., Cruz, J. L., \& Andres, M. V. (2013). Photonic fractional Fourier transformer with a single dispersive device. Optics Express, 21, 8558-8563. https://doi.org/10.1364/OE.21.008558.

Cuadrado-Laborde, C., Carrascosa, A., Pérez-Millán, P., Díez, A., Cruz, J. L., \& Andres, M. V. (2014). Phase recovery by using optical fiber dispersion. Optics Letters, 39, 598-601. https://doi.org/10.1364/OL.39.000598.

Cuadrado-Laborde, C., Costanzo-Caso, P., Duchowicz, R., \& Sicre, E. E. (2007). Periodic pulse train conformation based on the temporal Radon-Wigner transform. Optics Communications, 275, 94-103. https://doi.org/10.1016/j.optcom.2007.03.021.

Cuadrado-Laborde, C., Lancis, J., \& Andrés, M. V. (2010). Proposal of time-resolved chirpmeasurement through all-optical in-fiber mathematical operators. Optical and Quantum Electronics, 42, 97-108. https://doi.org/10.1007/s11082-010-9422-y.

Das, S. (2011). Functional fractional calculus. The Netherlands: Springer.

Davis, J. A., McNamara, D. E., \& Cottrell, D. M. (1998). Analysis of the fractional Hilbert transform. Applied Optics, 37, 6911-6913. https://doi.org/10.1364/AO.37.006911.

Emmerson, G. D., Watts, S. P., Gawith, C. B. E., Albanis, V., Ibsen, M., Williams, R. B., et al. (2002). Fabrication of directly UV-written channel waveguides with simultaneously defined integral Bragg gratings. Electronics Letters, 38, 1531-1532. https://doi. org/10.1049/el:20021056.

Erdogan, T. (1997). Fiber grating spectra. Journal of Lightwave Technology, 15, 1277-1294. https://doi.org/10.1109/50.618322.

Feced, R., \& Zervas, M. N. (2000). Efficient inverse scattering algorithm for the design of grating-assisted codirectional mode couplers. Journal of the Optical Society of America A, 17, 1573-1582. https://doi.org/10.1364/JOSAA.17.001573.

Feced, R., Zervas, M. N., \& Muriel, M. A. (1999). An efficient inverse scattering algorithm for the design of nonuniform fiber Bragg gratings. IEEE Journal of Quantum Electronics, 35, 1105-1115. https://doi.org/10.1109/3.777209.

Fernández-Ruiz, M. R., Carballar, A., \& Azaña, J. (2014). Arbitrary time-limited optical pulse processors based on transmission Bragg gratings. IEEE Photonics Technology Letters, 26, 1754-1757. https://doi.org/10.1109/LPT.2014.2332102.

Fernández-Ruiz, M. R., Wang, L., Carballar, A., Burla, M., Azaña, J., \& LaRochelle, S. (2015). THz-bandwidth photonic Hilbert transformers based on fiber Bragg gratings in transmission. Optics Letters, 40, 41-44. https://doi.org/10.1364/OL.40.000041.

Ferrera, M., Park, Y., Razzari, L., Little, B. E., Chu, S. T., Morandotti, R., et al. (2011). Alloptical 1st and 2nd order integration on a chip. Optics Express, 19, 23153-23161. https:// doi.org/10.1364/OE.19.023153.

Ge, J., Wang, C., \& Zhu, X. (2011). Fractional optical Hilbert transform using phase shifted fiber Bragg gratings. Optics Communications, 284, 3251-3257. https://doi.org/10.1016/j. optcom.2011.03.041.

Gerchberg, R. W., \& Saxton, W. O. (1972). A practical algorithm for the determination of the phase from image and diffraction plane pictures. Optik, 35, 237-246. 
Gopakumar, V. T., \& Madhusoodhanan, K. N. (2017). Design of all-optical integrators and differentiators using Fabry-Perot filters based on fiber Bragg gratings. Photonics Network Communications, 33, 377-388. https://doi.org/10.1007/s11107-016-0655-y.

Hahn, S. L. (2000). In A. D. Poularikas (Ed.), The transforms and applications handbook. (2nd ed.). Boca Raton: CRC Press.

Han, Y.-G., Kim, S. H., Lee, S. B., Paek, U.-C., \& Chung, Y. (2003). Development of core mode blocker with H2-loaded Ge-B codoped fibres. Electronics Letters, 39, 1107-1108. https://doi.org/10.1049/el:20030721.

Han, Y., Li, Z., Pan, S., Li, M., \& Yao, J. (2011). Photonic-assisted tunable microwave pulse fractional Hilbert transformer based on a temporal pulse shaping system. IEEE Photonics Technology Letters, 23, 570-572. https://doi.org/10.1109/LPT.2011.2116113.

Hinton, K. (1998). Dispersion compensation using apodized Bragg fiber gratings in transmission. Journal of Lightwave Technology, 16, 2336-2346.

Hua, J., Liu, L., \& Li, G. (1997). Observing the fractional Fourier transform by free-space Fresnel diffraction. Applied Optics, 36, 512-513. https://doi.org/10.1364/AO.36.000512.

Iaconis, C., \& Walmsley, I. A. (1998). Spectral phase interferometry for direct electric-field reconstruction of ultrashort optical pulses. Optics Letters, 23, 792-794. https://doi.org/ 10.1364/OL.23.000792.

Kane, D. J., \& Trebino, R. (1993). Characterization of arbitrary femtosecond pulses using frequency-resolved optical gating. IEEE Journal of Quantum Electronics, 29, 571-579. https://doi.org/10.1109/3.199311.

Kastler, A. (1950). A system of high-contrast diffraction fringes. Review Optik, 29, 307-314.

Kogelnik, H. (1976). Filter response of nonuniform almost-periodic structures. The Bell System Technical Journal, 55, 109-126. https:// doi.org/10.1002/j.1538-7305.1976.tb02062.x.

Kutay, A., Ozaktas, H. M., Ankan, O., \& Onural, L. (1997). Optimal filtering in fractional Fourier domains. IEEE Transactions on Signal Processing, 45, 1129-1143. https://doi.org/ $10.1109 / 78.575688$.

Lancis, J., Szoplik, T., Tajahuerce, E., Climent, V., \& Fernández-Alonso, M. (1997). Fractional derivative Fourier plane filter for phase-change visualization. Applied Optics, 36, 7461-7464. https://doi.org/10.1364/AO.36.007461.

Lee, B. H., \& Nishii, J. (1999). Dependence of fringe spacing on the grating separation in a long-period fiber grating pair. Applied Optics, 38, 3450-3459. https://doi.org/10.1364/ AO.38.003450.

Li, Z., Chi, H., Zhang, X., \& Yao, J. (2011). A continuously tunable microwave fractional Hilbert transformer based on a photonic microwave delay-line filter using a polarization modulator. IEEE Photonics Technology Letters, 23, 1694-1696. https://doi.org/10.1109/ LPT.2011.2166255.

Li, M., Shao, L. Y., Albert, J., \& Yao, J. (2011). Continuously tunable photonic fractional temporal differentiator based on a tilted fiber Bragg grating. IEEE Photonics Technology Letters, 23, 251-253. https://doi.org/10.1109/LPT.2010.2098475.

Li, M., \& Yao, J. (2010a). All-fiber temporal photonic fractional Hilbert transformer based on a directly designed fiber Bragg grating. Optics Letters, 35, 223-225. https://doi.org/10. 1364/OL.35.000223.

Li, M., \& Yao, J. (2010b). Experimental demonstration of a wideband photonic temporal Hilbert transformer based on a single fiber Bragg grating. IEEE Photonics Technology Letters, 22, 1559-1561. https://doi.org/10.1109/LPT.2010.2066964.

Li, Z., Zhang, S., Molina Vázquez, J., Liu, Y., Khoe, G. D., Dorren, H. J. S., et al. (2006). In Ultrafast optical differentiators based on asymmetric Mach-Zehnder interferometer. Proceedings symposium IEEE/LEOS Benelux chapter, Eindhoven (pp. 173-176).

Liu, B., Sima, C., Yang, W., Liu, D., Yu, Y., Gates, J., et al. (2015). In Realization of Bragg grating based integrated fractional photonic Hilbert transformer. 11th conference on lasers 
and electro-optics pacific rim (CLEO-PR) (pp. 1-2). https://doi.org/10.1109/CLEOPR. 2015.7376550.

Liu, W., Zhang, W., \& Yao, J. (2017). Silicon-based integrated tunable fractional order photonic temporal differentiators. Journal of Lightwave Technology, 35, 2487-2493. https:// doi. org/10.1109/JLT.2017.2688468.

Loh, W. H., Cole, M. J., Zervas, M. N., Barcelos, S., \& Laming, R. I. (1995). Complex grating structures with uniform phase masks based on the moving fiber-scanning beam technique. Optics Letters, 20, 2051-2053. https://doi.org/10.1364/OL.20.002051.

Lohmann, A. W. (1993). Image rotation, Wigner rotation, and the fractional Fourier transform. Journal of the Optical Society of America A, 10, 2181-2186. https://doi.org/10.1364/ JOSAA.10.002181.

Lohmann, A. W., \& Mendlovic, D. (1994). Fractional Fourier transform: Photonic implementation. Applied Optics, 33, 7661-7664. https://doi.org/10.1364/AO.33.007661.

Lohmann, A. W., Mendlovic, D., \& Zalevsky, Z. (1996). Fractional Hilbert transform. Optics Letters, 21, 281-283. https://doi.org/10.1364/OL.21.000281.

Lohmann, A. W., Mendlovic, D., \& Zalevsky, Z. (1998). IV: Fractional transformations in optics. Progress in Optics, 38, 263-342. https://doi.org/10.1016/S0079-6638(08) 70352-4.

Lohmann, A. W., Ojeda-Castañeda, J., \& Diaz-Santana, L. (1996). Fractional Hilbert transform: Optical implementation for 1-D objects. Optical Memory Neural Networks, 5, 131-135.

Lohmann, A. W., Tepichín, E., \& Ramírez, J. G. (1997). Optical implementation of the fractional Hilbert transform for two-dimensional objects. Applied Optics, 36, 6620-6626. https://doi.org/10.1364/AO.36.006620.

Lohmann, A. W., Zalevsky, Z., Dorsch, R. G., \& Mendlovic, D. (1998). Experimental considerations and scaling property, of the fractional Fourier transform. Optics Communications, 146, 55-61. https://doi.org/10.1016/S0030-4018(97)00521-X.

Lozovoy, V. V., Pastirk, I., \& Dantus, M. (2004). Multiphoton intrapulse interference. IV. Ultrashort laser pulse spectral phase characterization and compensation. Optics Letters, 29, 775-777. https://doi.org/10.1364/OL.29.000775.

Manabe, S. (1961). The non-integer integral and its application to control systems. English Translation Journal of Japan, 6, 83-87.

Mendlovic, D., \& Ozaktas, H. M. (1993a). Fractional Fourier transforms and their optical implementation: I. Journal of the Optical Society of America A, 10, 1875-1881. https:// doi.org/10.1364/JOSAA.10.001875.

Mendlovic, D., \& Ozaktas, H. M. (1993b). Fractional Fourier transforms and their optical implementation: II. Journal of the Optical Society of America A, 10, 1875-1881. https:// doi.org/10.1364/JOSAA.10.002522.

Miller, K. S., \& Ross, B. (1993). An introduction to the fractional calculus and fractional differential equations. New York: Wiley.

Muriel, M. A., Azaña, J., \& Carballar, A. (1999). Real-time Fourier transformer based on fiber gratings. Optics Letters, 24, 1-3. https://doi.org/10.1364/OL.24.000001.

Namias, V. (1980). The fractional order Fourier transform and its application to quantum mechanics. Journal of Applied Mathematics, 25, 241-265. https://doi.org/10.1093/ imamat/25.3.241.

Oldham, K. B., \& Spanier, J. (1974). The fractional calculus. San Diego, CA: Academic Press.

Oppenheim, A. V., \& Lim, J. S. (1981). The importance of phase in signals. Proceedings of the IEEE, 69, 529-541. https://doi.org/10.1109/PROC.1981.12022.

Ozaktas, H. M., Arık, S. Ö., \& Coşkun, T. (2011). Fundamental structure of Fresnel diffraction: Natural sampling grid and the fractional Fourier transform. Optics Letters, 36, 2524-2526. https://doi.org/10.1364/OL.36.002524. 
Ozaktas, H. M., Zalevsky, Z., \& Kutay, M. A. (2001). The fractional Fourier transform: With applications in optics and signal processing. Chichester: Wiley.

Park, Y., Ahn, T.-J., \& Azaña, J. (2008). Stabilization of a fiber-optic two-arm interferometer for ultra-short pulse signal processing applications. Applied Optics, 47, 417-421. https:// doi.org/10.1364/AO.47.000417.

Park, Y., Azaña, J., \& Slavík, R. (2007). Ultrafast all-optical first- and higher-order differentiators based on interferometers. Optics Letters, 32, 710-712. https://doi.org/ 10.1364/OL.32.000710.

Pellat-Finet, P. (1994). Fresnel diffraction and the fractional-order Fourier transform. Optics Letters, 19, 1388-1390. https://doi.org/10.1364/OL.19.001388.

Poladian, L. (2000). Simple grating synthesis algorithm. Optics Letters, 25, 787-789. https:// doi.org/10.1364/OL.25.000787.

Poveda-Wong, L., Carrascosa, A., Cuadrado-Laborde, C., Cruz, J. L., Díez, A., \& Andrés, M. V. (2016a). Experimental demonstration of fractional order differentiation using a long-period grating-based in-fiber modal interferometer. Optics Communications, 380, 35-40. https://doi.org/10.1016/j.optcom.2016.05.083.

Poveda-Wong, L., Carrascosa, A., Cuadrado-Laborde, C., Cruz, J. L., Díez, A., \& Andrés, M. V. (2016b). Long-period grating assisted fractional differentiation of highly chirped light pulses. Optics Communications, 363, 37-41. https://doi.org/10.1016/j. optcom.2015.10.063.

Poveda-Wong, L., Carrascosa, A., Cuadrado-Laborde, C., Cruz, J. L., Díez, A., \& Andrés, M. V. (2016c). Instantaneous frequency measurement by in-fiber 0.5 th order fractional differentiation. Optics Communications, 371, 89-92. https://doi.org/10.1016/ j.optcom.2016.03.061.

Rutman, R. S. (1995). On physical interpretations of fractional integration and differentiation. Theoretical and Mathematical Physics, 105, 393-404. https://doi.org/10.1007/ BF02070871.

Sabatier, J., Agrawal, O. P., \& Tenreiro Manchado, J. A. (Eds.), (2007). Advances in fractional calculus, theoretical developments and applications in physics and engineering. The Netherlands: Springer.

Schnebelin, C., \& de Chatellus, H. G. (2017). Fractional Fourier transform-based description of the Talbot effect: Application to analog signal processing. Applied Optics, 56, A62-A68. https://doi.org/10.1364/AO.56.000A62.

Shahoei, H., Albert, J., \& Yao, J. (2012). Tunable fractional order temporal differentiator by optically pumping a tilted fiber Bragg grating. IEEE Photonics Technology Letters, 24, 730-732. https://doi.org/10.1109/LPT.2012.2187331.

Shahoei, H., Li, M., \& Yao, J. (2011). Continuously tunable time delay using an optically pumped linear chirped fiber Bragg grating. Journal of Lightwave Technology, 29, 1465-1472. https://doi.org/10.1109/JLT.2011.2132754.

Shahoei, H., Xu, D. X., Schmid, J. H., \& Yao, J. (2013). Photonic fractional-order differentiator using an SOI microring resonator with an MMI coupler. IEEE Photonics Technology Letters, 25, 1408-1411. https://doi.org/10.1109/LPT.2013.2266252.

Sima, C., Gates, J. C., Zervas, M. N., \& Smith, P. G. R. (2013a). In Bragg grating based integrated photonic Hilbert transformers. 2013 IEEE international topical meeting on microwave photonics (MWP), Alexandria, VA (pp. 96-99). https://doi.org/10.1109/MWP.2013. 6724028.

Sima, C., Gates, J. C., Zervas, M. N., \& Smith, P. G. R. (2013b). Review of photonic Hilbert transformers. Frontiers of Optoelectronics, 6, 78-88. https://doi.org/10.1007/ s12200-012-0299-1.

Skaar, J. (2001). Synthesis of fiber Bragg gratings for use in transmission. Journal of the Optical Society of America A, 18, 557-564. https://doi.org/10.1364/JOSAA.18.000557. 
Skaar, J., Wang, L., \& Erdogan, T. (2001). On the synthesis of fiber Bragg gratings by layer peeling. IEEE Journal of Quantum Electronics, 37, 165-173. https:// doi.org/10.1109/3.903065.

Slavík, R., Kulishov, M., Park, Y., \& Azaña, J. (2009). Long-period-fiber-grating-based filter configuration enabling arbitrary linear filtering characteristics. Optics Letters, 34, 1045-1047. https://doi.org/10.1364/OL.34.001045.

Slavík, R., Park, Y., Krčmařík, D., \& Azaña, J. (2009). Stable all-fiber photonic temporal differentiator using a long-period fiber grating interferometer. Optics Communications, 282, 2339-2342. https:// doi.org/10.1016/j.optcom.2009.02.069.

Slavík, R., Park, Y., Kulishov, M., Morandotti, R., \& Azaña, J. (2006). Ultrafast all-optical differentiators. Optics Express, 14, 10699-10707. https://doi.org/10.1364/OE.14.010699.

Sun, S., Deng, Y., Huang, N., Tang, J., Zhu, N., \& Li, M. (2016). A tunable photonic temporal integrator with ultra-long integration time windows based on Raman-gain assisted phase-shifted silicon Bragg gratings. Optics Communications, 373, 91-94. https://doi.org/ 10.1016/j.optcom.2015.08.073.

Takyar, M. S., \& Georgiou, T. T. (2007). In The fractional integrator as a control design element. 46th IEEE conference on decision and control, New Orleans, LA (pp. 239-244). https://doi. org/10.1109/CDC.2007.4434934.

Torre, A. (2002). The fractional Fourier transform and some of its applications to optics. Progress in Optics, 43, 531-596. https://doi.org/10.1016/S0079-6638(02)80031-2.

Trebino, R. (2002). Frequency-resolved optical gating: The measurement of ultrashort laser pulses. Boston: Kluwer Academic Publishers.

van Howe, J., \& Xu, C. (2006). Ultrafast optical signal processing based upon space-time dualities. Journal of Lightwave Technology, 24, 2649-2662. https://doi.org/10.1109/JLT. 2006.875229.

Wolter, H. (1950). The minimum beam labelling as a means of increasing the accuracy of optical measurements and as a methodological tool for the replacement of the concept of the beam. Annalen der Physik, 442, 341-368.

Yamada, M., \& Sakuda, K. (1987). Analysis of almost-periodic distributed feedback slab waveguides via a fundamental matrix approach. Applied Optics, 26, 3474-3478. https://doi.org/10.1364/AO.26.003474.

Yariv, A. (2000). Universal relations for coupling of optical power between microresonators and dielectric waveguides. Electronics Letters, 36, 321-322. https://doi.org/10.1049/ el:20000340.

Zhang, X., Huo, T., Wang, C., Liao, W., Chen, T., Ai, S., et al. (2016). Optical computing for optical coherence tomography. Scientific Reports, 6, 37286. https://doi.org/10.1038/srep37286.

Zheng, A., Dong, J., Zhou, L., Xiao, X., Yang, Q., Zhang, X., et al. (2014). Fractional-order photonic differentiator using an on-chip microring resonator. Optics Letters, 39, 6355-6358. https://doi.org/10.1364/OL.39.006355.

Zheng, A., Yang, T., Xiao, X., Yang, Q., Zhang, X., \& Dong, J. (2014). Tunable fractionalorder differentiator using an electrically tuned silicon-on-isolator Mach-Zehnder interferometer. Optics Express, 22, 18232-18237. https://doi.org/10.1364/OE.22.018232. 